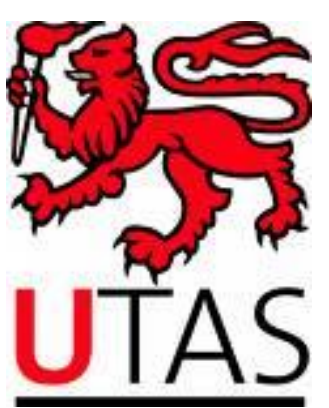

\title{
Exposure and Effect: An Investigation into a Culture of Body Pedagogies
}

\author{
by \\ Jennifer Ann McMahon \\ B.Ed. (Hons)
}

Submitted in fulfilment

of the requirements of the degree of

Doctor of Philosophy

Faculty of Education

University of Tasmania

Launceston

November 2010 


\section{Statement of originality}

I certify that this thesis contains no material which has been accepted for the award of any other degree or diploma by the University or any other institution, except by way of background information and duly acknowledged in the thesis, and to the best of my knowledge and belief it contains no material previously published or written by another person except where due acknowledgement is made in the text of thesis.

\section{Signature}

Jennifer Ann McMahon

Date 


\section{Statement of authority to access}

This thesis may be reproduced, archived, and communicated in any material form in whole or in part by the University of Tasmania or its agents, and may be made available for loan and copying in accordance with the Copyright Act 1968.

\section{Signature}

Jennifer Ann McMahon

Date 


\section{List of publications}

The author acknowledges that the following papers derive from this $\mathrm{PhD}$ thesis.

McMahon, J., Penney, D., \& Dinan-Thompson, M. (in press). Body practices Exposure and effect of a sporting culture? Stories from three Australian swimmers. Sport, Education and Society.

McMahon, J., \& Penney, D. (under review). Narrative ethnography and autoethnography: Empowering athletes and their bodies in and through research. Qualitative Research in Sport and Exercise.

\section{Signature}

Jennifer Ann McMahon

Date 


\begin{abstract}
This study sought to identify and articulate the exposure and effect of body pedagogies experienced by three former swimmers that participated in the amateur and elite cultural context of Australian swimming. Through the utilisation of 'relational analysis' (Kirk, 1999) and by 'connecting the dots' (Klein, 2000), investigation into the body pedagogies of three participants articulates two distinctive time frames - the participants as adolescents and some 10-30 years later, the same three participants as adult women enabling a link to be drawn between the exposure and effect of body pedagogies. Across both time frames, cultural transmission and lived bodily experiences are explored and fore grounded.
\end{abstract}

The research utilised narrative ethnography and autoethnography as it provided us [myself and the other two participants] with the appropriate space to reveal our knowing and detail our lived experiences. Four important considerations were integral to this research and to the use of narrative ethnography and autoethnography. The first consideration sought to foreground the swimmers' voice and the body's voice in the research. Another consideration was to enable the participants to have what I see as 'meaningful participation' in the research, positioning them in a genuine (rather than tokenistic) collaborative relationship with me as the researcher. Consideration was also given to the audience, with the intention that the reader would be able to take on and read from the positions and perspectives of the participants. Finally, I wanted the participants to gain some purpose from their involvement, gaining new insight with the intention of achieving 'order' in relation to their swimmer and bodily experiences.

A somewhat eclectic body of literature, drawn from various fields in regard to the body, culture, power and control has been utilised as the basis of a framework for understanding the complexities of lived experiences within a sporting culture. The literature review features the work of Foucault (1977) and Evans' (2004) work on the body together with others (Bain, 1990; Bordo, 1989, 
1992, 1993, 1997; Garrett, 2004; Jones, Armour \& Potrac, 2002; Johns \& Johns, 2000; Kirk \& Tinning, 1990; Pillow, 1997; Sparkes, 2004) and is designed to orientate the reader to the issues and questions at the heart of the research reported in this thesis.

When articulating the exposure of body pedagogies, the adolescent bodily experiences within the cultural context of Australian swimming were organised into two distinctive themes - [bodily] perfection which features in Act one of the thesis and performance which is the focus of Act two. The stories reveal body pedagogies occurring through practices within discourses of performance and perfection during the three participants' adolescence within the cultural context of Australian swimming. Act three explores the bodily experiences of the same three participants, in the present day as adult women, some 10-30 years after they first were exposed to and engaged with body pedagogies as adolescent women. In this section, the research investigated whether the body pedagogies that the three participants were exposed to as adolescents are still engaged with as adults. It also pursued the discourses underpinning and legitimated by the body pedagogies and examined whether or not those were subsequently challenged. Experiences and effects post-career, in adult lives revealed that the three participants embodied a damaged swimmer identity. It is 10-30 years on that the damaged swimmer identity continues to pervade their adult bodies and selves as they continue to engage in body pedagogies.

Prominent threads, tensions and themes were highlighted from the stories including; technocentric ideologies, slim to win ideologies, differential power relationships, self-surveillance, disciplinary power, discourses of performance and perfection relating to the body and performance, and meritocratic ideologies. Findings suggest that there is a definite connection between exposure and effect of a culture and that within the discourses of performance and perfection, a number of social and emotional issues are created which pervade the three participants' lives in both the short term and long term. The Australian swimming culture as represented in these lived experiences currently produces a 'brand' of swimmer formed through 'pedagogy,' 'traditions,' 'practices' and 'coach education.' This thesis proposes that the process and specifically body pedagogies involved in 
forming this 'brand' within discourses of performance and perfection, needs to be questioned and adjusted to consider the long term wellbeing of athletes. 


\section{Gratitudes}

\section{Associate Professor Dawn Penney}

My primary supervisor, good friend, mentor and supporter.

Without your support, dedication and commitment, I could not have made this dream possible.

I have learnt so much from you - we make a brilliant team.

\section{Dr Maree Dinan-Thompson}

My associate supervisor.

We started this journey together in 2007 at James Cook University.

I am honoured and grateful to have finished it with you.

\section{Dr Dean Cooley}

My associate supervisor.

A valued mentor and colleague.

\section{Chris, Finn and Zane Zehntner}

The three most valued boys in my life.

The three loves of my life.

My rocks.

Dr Pauline Taylor, Dr Linda Maloney, Angela Kershaw, Emma Roberts-Smith, Matthew Chappel, Dr Tammy Jones, Ms Robyn McCarthy, Dr Tim Moss, Dr Sharon Pittaway, Mrs Helen Yost \& numerous others

Friends, companions and my wider support network.

Without you, your support and your sense of humour,

this journey would not have been completed with a smile.

I promise to ring you more often now this is over.

\section{Australian Postgraduate Award Scheme and Faculty of Education, University} of Tasmania

For co-funding a scholarship for me

which has relieved some financial pressure during this journey. 


\section{Table of contents}

Statement of originality ................................................................................................................... ii

Statement of authority to access ...............................................................................ii

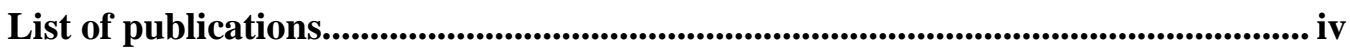

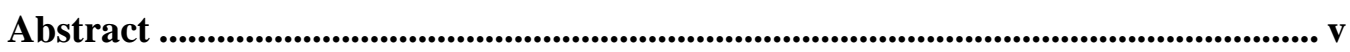

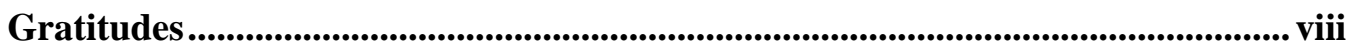

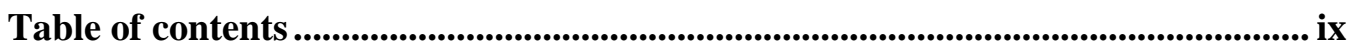

Table of tables .............................................................................................................

Exposition

Error! Bookmark not defined.

A story from July 1999 .......................................................Error! Bookmark not defined.

My journey: Calling upon the self......................................Error! Bookmark not defined.

Chapter One .................................................................. Error! Bookmark not defined.

Thesis structure..................................................................Error! Bookmark not defined.

Introducing the study ...........................................................Error! Bookmark not defined.

Introducing the actors (participants) ................................Error! Bookmark not defined.

Research questions................................................................Error! Bookmark not defined.

Chapter Two........................................................... Error! Bookmark not defined.

Situating the swimmers' voices: Investigating the journeys of others ................Error! Bookmark not defined.

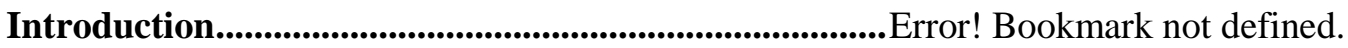

Body pedagogies.................................................................Error! Bookmark not defined.

Discourses of performance and perfection .....................Error! Bookmark not defined.

The aquatic back drop - the Panopticon ........................Error! Bookmark not defined.

Surveillance (gaze) ............................................................Error! Bookmark not defined.

Docile bodies.............................................................................Error! Bookmark not defined.

Normalisation ...................................................................Error! Bookmark not defined.

Situating the [swimmer] body within a culture...............Error! Bookmark not defined.

Conclusion .........................................................................Error! Bookmark not defined.

Chapter Three ........................................................... Error! Bookmark not defined.

Narrative ethnography and autoethnography: Empowering athletes and their

bodies in and through research ..........................................Error! Bookmark not defined.

Introduction........................................................................Error! Bookmark not defined.

A telephone conversation from January 2008.............Error! Bookmark not defined.

Selecting and shaping the research methodology with specific agendas............Error!

Bookmark not defined.

Employing narrative ethnography and autoethnography ..........Error! Bookmark not defined.

Constructing, re-constructing and (re)presenting stories ...........Error! Bookmark not defined.

Presentational form: Voices and vignettes .....................Error! Bookmark not defined.

Fore grounding the swimmers and their body's voice..Error! Bookmark not defined.

The individual and collective voice...................................Error! Bookmark not defined. 
Power-relations, empowerment and emancipation .......Error! Bookmark not defined. Clarity and meaning Error! Bookmark not defined.

Conclusion Error! Bookmark not defined.

Chapter Four Error! Bookmark not defined.

ACT 1 Error! Bookmark not defined.

Talking Swimmer Bodies: Exposure to a Body Focused Culture?............ Error! Bookmark not defined.

ACT ONE - Prequel ..Error! Bookmark not defined.

ACT 2. Error! Bookmark not defined.

Talking Swimmer Bodies: Exposure to a Performance Focused Culture?

Error! Bookmark not defined. ACT TWO - Prequel .Error! Bookmark not defined.

ACT 3. Error! Bookmark not defined.

\section{Talking Adult Bodies: Effects of a Performance and Perfection Focused} Culture? Error! Bookmark not defined.

ACT THREE - Sequel Error! Bookmark not defined.

Chapter Five Error! Bookmark not defined.

The critique Error! Bookmark not defined.

Introduction Error! Bookmark not defined.

Discourses of performance and perfection Error! Bookmark not defined.

Perfection Error! Bookmark not defined.

Performativity ..Error! Bookmark not defined.

Reproduction of the discourses of perfection and performance: the sites of learning Error! Bookmark not defined.

Disciplinary Power. Error! Bookmark not defined.

Panoptocism Error! Bookmark not defined.

Self surveillance. Error! Bookmark not defined.

Technologies of the self ..Error! Bookmark not defined.

Differential Power ..Error! Bookmark not defined.

10-30 years on: The effect Error! Bookmark not defined.

Subjects of our own surveillance ..Error! Bookmark not defined.

Fractured bodies ..Error! Bookmark not defined.

Conclusion ..Error! Bookmark not defined.

Chapter Six Error! Bookmark not defined.

Conclusion Error! Bookmark not defined.

Research significance: Revealing my learning - Act One and Act Two ............Error! Bookmark not defined.

Research significance: Revealing my learning - Act Three........Error! Bookmark not defined.

Recommendations for future research ..Error! Bookmark not defined. Ruminations of a journey ..Error! Bookmark not defined. Closing thoughts in relation to the research questions.Error! Bookmark not defined.

References Error! Bookmark not defined. 
Appendixes ........................................................................ Error! Bookmark not defined. Appendix A: Ethical Approval............................... Error! Bookmark not defined. Appendix B: Ethical Amendment ............................ Error! Bookmark not defined. Appendix C: Information Sheet ............................ Error! Bookmark not defined. Appendix D: Consent Form.................................... Error! Bookmark not defined. Appendix E: Counselling Advice ............................ Error! Bookmark not defined. 


\section{Table of tables}

Figure 1 - Visual representation of thesis structure.......................................................16 
"Even though my body seems the most private and hidden part of me, I carry my life history in my body, almost like the way the age rings of a sawn tree trunk reveal the process through time. My personal history of social practices and relationships is physically embodied in the customary ways I hold my body, imagine its size and shape, and in its daily movements and interactions"

(Jackson, 199o, p. 48). 
"Even though my body seems the most private and hidden part of me, I carry my life history in my body, almost like the way the age rings of a sawn tree trunk reveal the process through time. My personal history of social practices and relationships is physically embodied in the customary ways I hold my body, imagine its size and shape, and in its daily movements and interactions"

(Jackson, 199o, p. 48). 


\section{EXPOSITION $^{1}$}

${ }^{1}$ Exposition - The first occurrence of the theme or subject in a work, providing some background information to the readers about the emergence of the plot [theme]. 
"And with each day that passed, the void grew as my isolation became more accentuated. I wondered why my body was so troublesome. In such a situation, the discovery that my experience was not unique, that it had also been shared by others became very important for me" (McMahon, 2008). 
After ten or more years of isolating myself ${ }^{2}$ from anyone who was once part of the Australian swimming culture because of a hidden, and perhaps an unsubstantiated sense of shame, I started to re-connect with former team mates and competitors. Thanks to social networking sites, relationships that were once solid, before I consciously removed myself from the culture that I once strived to be a part of started to be rebuilt. Times that we once shared together as Australian swimming representatives were revisited and a camaraderie that an 'outsider' would find difficult to understand was rekindled. Profoundly, as my relationship with my former team mates grew, the hidden truths started to unfold.

I pondered when considering the topic for this thesis. It was only when I started to re-connect with my former team mates that I realised - my voice and the recollections that I revealed in the telling of my experiences as an Australian swimming representative in my honours thesis (McMahon, 2007) were actually relevant to others who were immersed in the sporting culture of swimming. It was then that I comprehended that I was not alone and that others had also struggled to maintain the ideal swimmer body. Below, I include a story which for the purpose of this thesis has been italicised, like the other stories told to indicate to the reader that it is a lived experience. The story that follows comes from my honours thesis, a story of experience that I shared with my former team mates, a story they were able to identify with and from this, the hidden truths and admissions started to unfold, that were not unlike my own.

\section{A story from July 1999}

It is $3 \mathrm{pm}$. I climb into my car to drive to training. U2, a personal favourite is playing on the radio. I feel invigorated and free. My life is purposeful again. I turn the volume up on my stereo and I sing at the top of my lungs. I am excited about going training. It feels as though butterflies are crazily buzzing around in

\footnotetext{
${ }^{2}$ Self has been italicised throughout this research as it denotes the importance of the body, being and self as one static entity. In so doing, self encompasses the union of all elements including; emotions, spirituality, thoughts, sensations, experiences, conversations and constitutes the individuality and identity of each person, as a soul, underlying all subjective experience.
} 
my stomach. I drive just over the speed limit to get there. I cannot wait to see my training partners.

I have an extra spring in my step as I walk into the pool. I have been swimming so fast in training. The head coach commented last week when he visited our squad. This morning, I was swimming out of my skin. I feel like I have an athletic purpose again. I no longer feel like a pretender. I am 25 and it has been 7 years since I have competed, but now, for the first time in years, I am excited about the possibilities that lay ahead.

I know I can swim faster than I used to. Am doing everything possible to make sure I do. Am going to a dietician to make sure my eating is sorted. Am going to sports psychologist to make sure my head is sorted. Going to make my mind and body stronger than ever before. Making the Olympic team is a real possibility everything is coming together nicely.

As I complete my stretching routine, joking and laughing with my team mates, my coach approaches me.

Coach: "Jenny, can I see you before you hop into the pool."

I am bursting with anticipation. I know he is going to say something about how hard I have been working. I bet he can see the changes - the changes in my body, the changes in my fitness - the changes in my speed. I hurriedly put on my bathing cap and grab my goggles because I can't wait to hear the good things that he has to say. As I approach him on pool deck, his expression is serious and suddenly a blanket of caution surrounds me.

Coach: "If you are serious about making that Olympic team next year, you are going to have to lose weight. You are carrying too much weight."

Warmness is absent from his voice. It is déjà vu. I have heard these types of comments many times before from him and other coaches on the Australian swimming teams. I want to reply. I bite down on my tongue as I have learnt to silence my voice, because a reply is usually met with disapproval. I bite down hard but I cannot help myself. 
Jenny: "What do you mean? I have been meeting with my dietician on a weekly basis for the past two months. We have made some real progress. I am losing weight each week."

Coach: "You need to lose more weight if you are serious, you can't swim fast with the weight that you are carrying."

Jenny: "But, I have lost 6 kilograms."

Coach: "It needs to be more; you are carrying too much weight on your bum."

It was as though a lightning bolt had struck me. My confidence is removed and replaced with self-doubt and self-loathing. It was like a click of the fingers or a flick of a switch. Suddenly, the session that lay ahead no longer seems exciting. I complete it in silence. My thoughts drift back to the 1992 Olympic trials when I missed the Australian team. My weight was blamed then for my failure to qualify. Anger fills my insides. Heaviness encompasses my body like a blanket has been placed across my back in the water. My team-mates gaze into my emotionless face with a wondering look as I am not my usual joking self.

I realise that my training efforts mean nothing to him if I do not look the part. I try to imagine what he wants me to look like, what the shape it is that he says will bring success - a body that will swim faster than the body that I have now. I thought that I had that shape, which I had worked so very hard to get it, broad shoulders with narrow hips.

Need to get narrower hips.

I envisage a silhouette; it is tall, willowy and ghost like in appearance. It doesn't have a face. The silhouette's hips, even though it is a female, resemble that of a male. This is what my body needs to be. Have been told, my bum is too fat.

Need to lose my bum fat.

I try and recall back, searching for a time when I was that body - when my body made him happy. I try to remember. It has been a long time - I was fifteen. 
The session is over and I wonder what the best way is to exit the pool without exposing myself. I feel ashamed. I don't want people to look at my fat body as I hop out. I do some extra laps while I think of the best way to get out. My teammates have hit the showers and my coach has his back turned and is talking to a parent of another swimmer.

This is my opportunity.

I dash out of the pool and grab my towel before he can see me, before anyone else can see me. I cover my body and find myself scanning the pool deck to see if anybody was watching me.

I am so ashamed of my body.

That night when I arrive home, I think about what my coach said to me - his disapproval of my swimmer body. I walk over to the fridge-opening it and staring at the contents inside wondering what to make for dinner. My hunger is not like it usually is after training, instead I feel full and food disinterests me. Instead of eating, I climb into my pyjamas and go to bed.

(McMahon, 2007)

The hidden truths that began to emerge from my former team mates once I shared this story with them disconcerted me. The stories that emerged ripped "open a scab that I hadn't known still lingered on my psyche, and I gasped as the blood/memory flowed" (Ellingson, 2005, p. 95). This was it - the pivotal moment in the conception of this thesis. It was the time when I realised that I was not alone in my bodily experiences and that many of my former team mates had also struggled with their swimmer bodies. Below I privilege five ${ }^{3}$ voices of former team mates - voices that fuelled my inquisitiveness to interrogate a culture that we once strived to be a part of, a culture that some 10 to 30 years on seemingly continues to pervade our bodies and lives.

"No one else knows this but I was bulimic. Regret everything but didn't know what else to do? Everyone always thought I had my weight under control."

\footnotetext{
${ }^{3}$ The voices of my former team mates are personally communicated to me. They have subsequently permitted me to use their voice/s in this thesis.
} 
"My god, I can relate to your story. I was told that I was fat at the age of 10. Like most swimmers, I did get fat when I quit, I don't say that I retiredI quit. You would never believe what I went through as a young kid. We need to get something out there to those idiots who put so much pressure on kids to succeed. I would love to help you by sharing my stories because, God the stories that I could tell you that's for sure but I don't want to bag my coach even though I spent most nights crying over him...I hope you understand?"

"Your paper was excellent; however it brought back suppressed memories and a lot of tears. I see this as good, as now I have had to face some extra issues."

"I remember way too vividly my experiences with my weight and always feeling fat even though I was quite obviously not (I can say that now because I have seen photos of myself during that time). One of the things that I can particularly remember is arriving at training and trying to either sneak the scales out of the cupboard so I could secretly weigh myself (just so I knew what to expect when it was done in front of everyone else) or if I could not get them, trying to guesstimate my weight with the lat pull down machine. The machine had weights set to 100 kilograms, and if I set the weights to my approximate weight (or what I was supposed to be) I could hang off the bar and know depending on how much I balanced or rose, as to what the result would be on the scales. It sounds like a simple and funny thing now, but it was terribly stressful at the time. I was constantly worried about what the scales and therefore my coach would say. I hated the public humiliation of putting on weight. I also remember that when I was 'light, 'I trained well. If I was 'heavy,' I felt depressed, usually got chastised or at the very least dirty looks or was ignored by my coach, and then trained poorly. It was not a mentally healthy way to live."

"I can remember one of the coaches on the Australian swimming team saying to me on the morning of my race that I looked like that I had put on weight. I thought to myself... what do you expect me to do about it now when my race is like only two hours away.... what a fool." 


\section{My journey: Calling upon the self}

\section{(The Then - XIV Commonwealth Games, New Zealand)}

Ray Warren: “McMahon, McMahon might run second! But, in goes Hayley, three gold medals, three for Hayley. And did McMahon beat Noall, yes she did! They've got the quinella ${ }^{4}$, this is incredible! This is incredible Australia, these kids, they have fair dinkum improved unbelievably since Adelaide."

Norman May: "Look at this, Hayley really gave them something, but further on this side, Jenny McMahon flew in the last five metres to get Noall. Noall looked across, she looked up and that is what cost her second place."

Ray Warren: “Jenny McMahon, she just keeps breaking her best, 2.02.43, it's about one and a half under!"

Norman May: "She has come down 1.31 seconds. That means in about five weeks, she has come down about four and a half, five seconds!"

Ray Warren: "Little Jenny McMahon, a 15-year-old quinella for Australia, Lewis 15, McMahon 15. And Jenny comes from the Woogaroo club. What a sensational swim, she has gone from rank nine to about rank three in just over two minutes. This is sheer excitement, I hope you are getting this loud and clear in Aussie land."

Norman May: "One, two for Australia, the only way that we can go better than that is a dead heat!"

Ray Warren: "I wish you could be here Australia."

Norman May: "I wish I could pick race horses like I could pick swimmers, if Jenny McMahon is not right up there in Barcelona Olympics in a few years time, then I am no judge of swimmers."

Ray Warren: "Ladies and Gentleman, I want to tell a story about Norman May. He has just given Jenny McMahon a huge wrap but he doesn't hand them out

\footnotetext{
${ }^{4}$ The term quinella refers to a form of gambling where the person who is betting picks the first and second place getter. The term is usually used in horse racing.
} 
loosely! First time I worked swimming was in Perth last August and we saw a little blonde headed fellow, who we call Queensland's Great White Shark Mark Two, Glen Housman, and after 50 metres, Norman said, he is a champion and he will break a world record, and you wouldn't believe it, just a few months later he did! I can tell that story whereas he wouldn't. Good on you Hayley and Jennifer!"

(Commentators, Norman May and Ray Warren. Channel nine's coverage of the Women's 200 metre freestyle at the XIV Commonwealth Games, $27^{\text {th }}$ January, 1990, Auckland: New Zealand).

Representing Australia in swimming and winning medals internationally was an extraordinary part of my life. As an adolescent, my entire identity was based on swimming fast and winning, it permeated my entire being. It was as though, the idea and goal of representing Australia at the Olympics ran through the blood in my veins; it was in every breath of air I took, every low fat carbohydrate that I ate and every kilometre that I swam. This was not always the case though. I recognise that it was something that developed as I did, physically and emotionally as a swimmer. Standing on the dais to receive a gold medal, watching the Australian flag being raised, with everyone in the crowd standing to attention while the national anthem was playing was the very essence of my existence as a teenager. It is what drove me to train six hours a day, six days a week for over ten years. I saw swimming as part of my entire being ${ }^{5}$ and making an Australian swimming team and hearing the national anthem play became the sole focus of my thoughts and behaviour. Winning a gold medal for Australia was for me the ultimate; it was the pinnacle of a long journey which began for me, without realising it, at nine years of age.

The journey is not always as successful. I was lucky. Many have gone before and after me, training six hours a day, for years on end, sacrificing their childhood and teenage years in pursuit of the dream of representing Australia and winning gold, which seemed to be sought after by all of those in the culture around me. These goals are always there, in every elite swimmer's mind,

\footnotetext{
${ }^{5}$ Body, self and being are used interchangeably throughout this thesis and encompass the elements as body, emotions, spirituality, thoughts, sensations, experiences, conversations and constitute the individuality and identity of each person, as a soul, underlying all subjective experience. Body, self and being have been formatted in italics to communicate to the reader the enormity of what I consider these terms to encompass for us as human beings.
} 
embedded in our bodies and our psyche, dangling like a carrot in front of a donkey, dictating ever lap, every set and every session of a swimmer's preparation. Sometimes the dream does not become reality, the goals are never attained and that feeling of standing on the dais is never experienced, not because of a lack of dedication, not because of a lack of talent but because of not getting it right on the day, whether that is due to an injury or sickness, the preparation given by the coach in the lead up to the competition or the $\operatorname{taper}^{6}$ that has been set.

The journey of a swimmer that I describe is just as long and arduous for the swimming coach. The swimming coach is a mentor, a guide, a facilitator, a surrogate parent to a swimmer who may spend up to six hours a day in their care, growing from a child into an adolescent and then through into an adult. Their dream is your dream and in some instances therefore, representing Australia becomes the very essence of a coach-swimmer relationship. I remember meeting my coach for the first time when I was three. Unlike other coaches, he also taught children to swim. It seemed I did not have a natural relationship with the water in the beginning and used to spend much of the lesson crying. I was a slow learner and I remember my constant crying frustrated my coach. I used to alternate swimming teachers each week between him and his daughter. It took five years before I learnt to do the four swimming stokes and before I felt comfortable in the water. My parents were worried that I might forget how to swim if I stopped so they enrolled me into a beginner training squad at eight years of age. This is when my relationship with my coach developed. I would only go to squad training once or twice a week. It was fun; he was fun. We got to do lots of technique drills and dives. I remember training to be a very social event during these early days.

At nine years of age, I swam at state titles. I cannot remember if it was my idea, my parents' or my coach's. I do remember that I was happy enough to go along and was fascinated by the Chandler Aquatic complex in Brisbane. The stands were full of people and everyone was cheering. I swam only one race, the 100 metres freestyle. I did not make the final. I do not remember being disappointed; more amazed at how fast the girls who were my age swam. I

\footnotetext{
${ }^{6}$ A taper in the context of sport refers to the practice of reducing, or tapering off training/workload in the days just before an important competition to enhance competitive performance. A taper is quite in-depth as each individual swimmer responds differently. Some swimmers only need a small decreasing in work load, while others can need a two week taper.
} 
remember wanting to be as fast as them and the swimming-racing environment for me, created a fire in my belly. As the years passed, my desire for racing increased. My coach was very good at putting things into perspective in my early years in the sport. School and family life always came before swimming. He always encouraged my family to go away on a holiday during the school break and I always had a couple of months break from training during the winter months. The early years in my swimming career were such fun and in a way, my coach was learning with me. He only really entered the profession when I went along to swimming lessons at the age of three. He had chosen, at 50 years of age, a complete career change. He was originally a plumber, then turned his hand to horse training and then went on to begin a swim school.

I never remember being pushed or having any unpleasant feelings in the sport except for once when I was disqualified by a carnival referee in a breaststroke race for doing an incorrect kick. When I turned 13, it seemed that, all of a sudden, everything clicked. I went from being a strong regional (Ipswich and Brisbane area in Queensland) swimmer who won the occasional state medal, to winning numerous gold medals at the national age titles. The national age title in Perth in 1989 marked this transition. I won three national age titles, becoming the national champion for my age. Immediately, I felt like I was cast into the limelight of the national age championships as coaches, parents and swimmers all began to not only notice me but pay attention to me. Without realising it at the time, this is when my relationship with the sport of swimming, with my coach and with my parents began to change. This was also the first time that I considered the possibility that I might one day make an Australian swimming team. I did not have a plan. I did not tell anybody. It was just a thought, a brief passing moment. At that stage, I had no idea what I had to do to make an Australian swimming team.

After the national age titles, I won an associate scholarship with the Australian Institute of Sport (AIS). The scholarship allowed me to remain with my own coach on a full-time basis, while visiting the Institute in Canberra for short periods of time, to be tested and to have my technique biomechanically examined by experts. My first visit to the AIS was shortly after the national titles. It was August 1989. I had blood lactate tests, pathology tests, urine tests and was 
filmed swimming, with detail shots taken of my technique both above and below the water. Up until that point, I was considered to be a backstroke and a medley swimmer. The tests revealed that I was physiologically suited to middle distance freestyle swimming. The coaches at the Institute were also very impressed with my training performance and the swimming times that I was holding in training sets. I liked the attention that I received from them and I seemed to thrive in the environment. The coaches from the Institute mentioned to me that I should go to the Australian open swimming titles in December, just three and a half months away. I did not even know that the competition was on, and up until that point, my sole focus had been national age titles. Competing at the national open championships had never crossed my mind. The championships were open, which meant that even though I was 15 years of age; I would be racing against girls and young women from every age group. The titles also doubled as trials for the Australian Commonwealth Games team to be held in Auckland, New Zealand. A few weeks earlier, I had a brief moment where I dreamt about making an Australian team, but by no means did I think that I would be good enough to be selected in the Australian Commonwealth Games team in just 12 weeks.

The three months passed quite quickly and before I knew it, December had come around. I travelled to Adelaide with two other training mates from my squad and the rest of the Queensland swimming team. I am not sure if my training partners were expecting to make the Australian team, as we had never discussed it with each other in training. Our conversations were mainly filled with what we had done at school, which girls they were intending to ask out and what they were doing on the weekend. I was not nervous about competing at the meet, probably because I had no expectation of how I would perform.

When I arrived at the pool where the titles were, I recognised a few familiar faces, those who had competed with me at the national age titles in Perth and some more prominent faces that I had seen competing for Australia on television. My main event was on the first day of competition. It was the 200 metres freestyle. I swam the heat with no expectations of making the final and to my surprise, I felt strong in the water. I recorded a 2.04 in the heat and qualified fourth fastest for the final. I did not have any nerves between the heat and final, as I still did not expect that I would medal. To my surprise and the surprise of others, 
my physical performance surpassed my mental expectations and I finished third in a time of 2.03.76. This was fast enough to qualify me for an individual berth in the $200 \mathrm{~m}$ freestyle event and a place in the $4 \times 200$ metre freestyle relay, representing Australia at the XIVth Commonwealth Games in Auckland, New Zealand.

I now realise that this is when my relationship with the sport changed dramatically due to my subsequent immersion in the elite culture. It is the time when I bought into the Olympic dream completely. Decisions in every aspect of my life all revolved around Olympic representation. The elite culture exposed me to practices and ideologies that still affect me now, some 20 years later. It is only recently that I came to realise that I was lucky, as for others it began earlier and like them, a troublesome swimmer body was created. This thesis reveals the stories of three swimmers and their exposure to a culture and the effects of such exposure. 
CHAPTER ONE 


\section{Thesis structure}

A theatrical approach has been utilised throughout this thesis as a method of arranging the sections. The Exposition commenced this research production by providing the reader with an opening, a prelude ${ }^{7}$ to the main performance. The Exposition contains an autobiographical account of my journey as an elite swimmer, as a means of locating myself in this research as well as the impetus of this production. Table one (below) further provides a visual representation of this thesis structure.

Chapter one introduces the thesis, including the structure of the thesis, details of the study, introduction of the actors [participants] of this production and the research questions.

Chapter two introduces the reader to a somewhat eclectic collection of literature. The chapter provides an analytical lens through which the reader will be able to "interrogate the recipes that a culture provides" (Sparkes, 1999, p. 26; see also Bruner, 1987), orientating them to the issues and questions at the heart of the research reported in this thesis.

In Chapter three, I describe how narrative research utilising an ethnographic framework and autoethnography evolved as optimal methodologies for this doctoral research project that has explored the experiences of three swimmers' bodily experiences in the elite and amateur sporting culture of Australian swimming.

Chapters four presents Act one, Act two and Act three. These acts make up the very core of this research production. This is the section where the participants take centre stage and their stories of experiences are shared.

The critique features in Chapter five. The stories of experience are discussed and the theoretical framework (Chapter two) is utilised as a method of analysis in order to address the research questions. Finally, Chapter six draws together and summarises this research production, particularly the findings in regard to the

\footnotetext{
${ }^{7}$ A prelude is an introductory performance, episode, or action preceding a more important one; a preliminary or preface to a production.
} 
research questions. Conclusions are drawn from the lived experiences of myself and the other two participants. In this chapter, the significance and potential contribution of the findings of this research will also be discussed. In closing, I provide a personal reflection of this research journey, summarising my learning throughout the research process.

THESIS STRUCTURE

Exposition

Introduction

Literature Review

Methodology

Act 1 (Prequel)

Body pedagogies: discourse of perfection
Act 2 (Prequel)

Body pedagogies: discourse of performance

\section{Act 3 (Sequel)}

Body Pedagogies as adults

\section{Critique}

Conclusion

\section{Table 1 - Thesis structure}

\section{Introducing the study}

In this thesis, I narrate the stories of myself and two other former swimmers. The intention in this research was to explore the exposure and effect of body pedagogies. Two distinctive themes have been identified in the data and subsequently, utilised as a method of organising the experiences of the swimmers; [bodily] perfection and performance. I use the concept of body pedagogies as a 
means of understanding how the discourses of [bodily] perfection and performance are shaping the understanding, practices and relationships that three swimmers have with their bodies both in the short term and also long term within the cultural context of Australian swimming.

Firstly, the body pedagogies that myself and the two other swimmers were exposed to as adolescents within the sporting culture of Australian swimming will be articulated. Secondly, the bodily experiences of the same three participants some 10-30 years after being immersed in the Australian swimming culture will be revealed. Examination of the discourses through which body pedagogies pervade lived adult experiences will be explored. Focus will be placed on whether in fact the body pedagogies and discourses that dominated adolescent experiences have subsequently been challenged. This is done by utilising 'relational analysis' (Kirk, 1999) and by 'connecting the dots' (Klein, 2000), exploring the "pedagogic palette" (Bernstein, 2000, p. 52) and whether it had an 'effect' on long term wellbeing.

\section{Introducing the actors (participants)}

Only two former swimmers from a number of both male and females that I approached permitted me to share their stories of experience in this thesis. The voices of others who I have been in communication with or have been involved with in some way have echoed similar sentiments to that which I revealed in my honours thesis. Those who declined to participate in this research production did so for various reasons. Some had an overwhelming sense of loyalty to their coach and expressed a fear of betraying them if they participated. They also feared having their identity exposed even after being assured that all identifiable information would be removed. Some clearly see an eating disorder to be a sign of weakness, and even though every swimmer that I have been involved with attributes their damaged swimmer body to the culture of the sport or a coach, they still feel an overwhelming sense of loyalty in protecting the culture and the coach. I express my sincere gratitude to the two women who had the strength to share their stories and participate in this research. While the three participants in this research are all women, this was not intentional as my desire was to include 
the voices of both male and females. From my own experiences as an elite swimmer, it was evident that body pedagogies were practiced on both male and female swimmers. Subsequently, I chose not to pursue the dimension of gender difference in the body pedagogies experienced. This is acknowledged as a potential line of inquiry for other future studies to pursue

/arly is a 34 year old secondary school teacher. She is also a wife and a
mother of two young children. Carly is a former state level competitive swimmer. Her stories of experience occurred over a five year period between the ages of ten and 15. After 'quitting' the sport, she has been unable to bring herself to go for a recreational swim, even for the fitness benefits that can be gained because she feels that there are too many bad memories associated with her involvement in the sport. She describes herself as competitive and hard working, constantly striving to be the best in all that she does. Her self esteem, for the past 23 years has been closely linked to her weight. Carly says that when she weighs light, she feels great, however when she weighs heavy, she falls apart emotionally. Swimming was an important part of her life growing up, in fact every decision that was made by her parents made swimming the first priority. She bought into the Olympic dream at seven years of age and so did her parents. Consequently, she did not have a normal childhood as it revolved around training hard, eating minimally and competing. Although Carly has not swum competitively for over 14 years, her swimmer identity is still strong. She still continues to define herself as an Olympic failure.

M andy is a 45 year old small business owner and a part time swimming coach. She is recently divorced and has no children. Mandy is a former national representative elite swimmer, having competed at the Olympic Games, Commonwealth Games and World Championships, breaking records and winning medals during this time. Her stories of experience that feature in Act one and Act two of this thesis occurred over a four year period between the ages of 14 and 18 . Like Carly, after Mandy retired from the sport, she no longer swam for recreation or for fitness benefits. Mandy describes herself as hard working but believes she lacks confidence. Mandy battled with bulimia for 31 years which she believes started because of two coaches on an Australian swimming team. She continued to be bulimic for 27 years after retiring from the sport. It is only in the last two 
years that with the proper help, she has started to conquer the disorder. Her parents provided her with a stable background and her life at home did not revolve around the sport. After retiring from the sport, she has been an owner of a small business and has also coached swimming, having been selected as an Australian representative coach several years ago. Currently she is involved with coaching age group swimmers.

$\underline{\underline{J}}$

(Jenny) am 35 years old. I work part time teaching at a University. I am a wife and mother of two young and very active boys. I am a former national representative elite swimmer, having represented Australia at the Commonwealth Games, World Championships as well as numerous World Cup meets. My stories of experience occurred over a 10 year period between the ages of 15 and 25 . After retiring from the sport, I have continued periodically to swim in an adult swimming squad although I did not swim for a period of time after having my children. My body has been troublesome since I was 16 years of age. I have had moments of extreme dieting, excessive exercising, abusing diet medication and laxatives as well as almost the complete opposite where I have had a period of time where I did no exercise whatsoever and consumed whatever I wanted.

\section{Research questions}

The overarching question for this thesis is:

- What are the effects of the body pedagogies of a culture?

The following questions supported me to further interrogate the culture of Australian swimming, contextualising the overarching research question:

- What body pedagogies within the discourse of perfection were each of the three swimmers exposed to as adolescents during their immersion in the Australian swimming culture?

- What body pedagogies within the discourse of performance were each of the three swimmers exposed to as adolescents during their immersion in the Australian swimming culture? 
- What body pedagogies do the three participants still engage with some 10-30 years after being immersed in the Australian swimming culture?

- Can a link be made between the exposure $e^{8}$ and effect ${ }^{9}$ of body pedagogies occurring within a culture?

\footnotetext{
${ }^{8}$ During our adolescence, body pedagogies were practiced on our bodies and subsequently engaged with by our selves demonstrating what I term 'exposure.'

${ }^{9}$ Some 10-30 years on as adult women, we have either continued to engage with body pedagogies or subsequently challenged revealing what I have termed 'effect' in this thesis.
} 
CHAPTER TWO 


\title{
Situating the swimmers' voices: Investigating the journeys of others
}

\author{
"The greater our knowledge increases, \\ the greater our ignorance unfolds." (John $\mathcal{F}$ Kennedy)
}

\section{Introduction}

This chapter provides the reader with an orientation to the core tenets of this thesis. The first section excavates body pedagogies and specifically explores culture, lived experience and embodiment, and discourses of performance and perfection (Evans, Rich, Davies, \& Allwood, 2008). This is done within the context of swimming, investigating the elite and amateur sub-contexts as well as the multiple sites of practices (Evans et al., 2008). Excavation of a somewhat eclectic body of literature, drawn from various fields in regard to the body, culture, power and control is presented as the basis of a framework for understanding the complexities of lived experiences within sporting cultures. The review aims to provide the reader with an analytical lens through which to "interrogate the recipes that a culture provides" (Sparkes, 1999, p. 26) and to orientate the reader to the issues and questions at the heart of the research reported in this thesis.

The next section explores operations relating to disciplinary power (Foucault, 1977), a concept that is identified as central to exploration of the lived experiences of athletes within sporting cultures and amidst pedagogical practices and relations associated with those cultures. The final section in the chapter engages the reader in literature relating to autoethnography and narrative ethnography (Gubrium \& Holstein, 1999; Tedlock, 1991) pertinent to sociocritical inquiries in the fields of sport and education. This section specifically points to the value and significance of storytelling in such research and highlights issues of complexity, rich potential and considerable challenges inherent in employing these methodologies with specific agendas. I found four considerations paramount in regard to methodology and in particular, the issue of empowerment 
of participants in and through the research process. These are detailed in Chapter three.

\section{Body pedagogies}

This thesis focuses on body pedagogies, with emphasis placed on the defining and shaping of particular bodies (Rich, 2010), cultural transmission, lived experience and embodied change (Evans et al., 2008). Rich and Evans (2007) describe body pedagogies as "any conscious activity taken by persons, organizations, or the state designed to enhance individuals' understandings of their own and others' corporeality $^{10 »}$ (p. 41). For Shilling (2007, p. 13), body pedagogies refer to:

the central pedagogic means through which a culture seeks to transmit its main corporeal techniques, skills, dispositions and beliefs, the embodied experiences typically associated with acquiring or failing to acquire these attributes, and the actual embodied changes resulting from this process.

Body pedagogies can thus be seen to exist and 'operate' across and through multiple sites of practice, defining the significance and potential of the body in time, place and space (Evans et al., 2008). From this perspective, body pedagogies are socially and culturally situated, reflecting the prevailing corporeal orientations and thus provide resources through which individuals come to understand their bodies in relation to the social and cultural contexts to which they are immersed (Evans et al., 2008).

In considering sporting cultures and contexts specifically, the multiple sites [places] of practice described by Evans et al. (2008), could include the training environment, competition sites and events, and home environment, while encompassing social interactions with coaches, peers and family members. Rich (2010, p. 147) further states:

It is now well recognised that pedagogical activity can no longer be considered as confined to contexts of formal learning (e.g., school or

${ }^{10}$ Tangible; physical; of or pertaining to the body; bodily; practices 
colleges) but instead occurs within many other socio-political and cultural sites, such as families, churches, mosques, amongst peers, and via sites of popular and consumer culture (i.e., the messages transmitted through TV, film, newspapers and magazines, etc.) often credentialed by media figures claiming particular expertise (e.g., around diet, exercise and health).

Vertinsky (2004, p. 9), explains that places [sites] are made "through power relations, which construct the rules, which define the boundaries; these boundaries are both social and spatial." As such, sporting bodies and sporting sites are woven together through intimate webs of social and spatial rules that are made by and make embodied sporting subjects (Vertinsky, 2004). A sporting site is thus "the body's playground" (Vertinsky, 2004, p. 22) through which bodies are encouraged to move and relate in prescribed ways. As such, the potentiality of a site can be recognised as it can be remembered, partly because it is unique, but also because it has affected our bodies and generated enough associations to hold it in an embodied way from which memory is carried away (Vertinsky, 2004).

Body pedagogics (Shilling, 2007; 2010) classify bodies of value and status by defining and positioning those bodies deemed to be more or less valuable within specific social and cultural contexts. For Giroux (1994), pedagogy is ultimately about the "creation of a public sphere through which the production of knowledge, identities, social relations and values that take place in a variety of cultural sites" (p. 155). When applying body pedagogics (Shilling, 2007) to sporting cultures, and specifically, elite sporting cultures, practices such as skin fold measurements, and practices focusing on weight, shape and performance, are pertinent. Drawing on Bernstein's (1996) work, such practices can be seen as both embedded with a regulatory dimension and simultaneously, as having a regulatory role; regulating the social interactions occurring within the different discourses that fall within the culture. As Evans et al. (2008, p. 17) explain, "body pedagogies construct particular social meanings which have an impact upon young people's identities" specifically in regard to their ability to conform to the ideal shape and also their ability to perform. Evans et al. (2008) discussed the power inherent in body pedagogies that was revealed through the voices of young people in their research. They illustrated the defining and normalising processes 
operating in and through body pedagogies, highlighting "particular dispositions and attitudes towards the body within a culture of performativity where people are subject to increasing pressures" (p. 17) from testing and assessment on the body. Evans et al. (2008, p. 98) thus emphasised, "all pedagogical relations are power relations; there is no instruction without regulation; no pedagogy is divorced from control".

Research and critique that addresses the long-term effects of body pedagogies, specifically in relation to the act of normalisation (Foucault, 1977) is still largely lacking in the social sciences. More specifically, the pedagogical practices and attitudes towards the body within cultures of 'performativity' and/or 'perfection' require further probing. This thesis attempts to excavate whether prejudice occurs in relation to those bodies that do not conform and the extent to which these cultural inscriptions pervade lived experience. Furthermore, it also examines the permanence of body pedagogies and the extent to which they pervade an athlete's self in the long term. I use the concept of body pedagogies as a means of understanding how the discourses of [bodily] perfection and performance are shaping the understanding, practices and relationships that three participants have with their bodies both in the short term and also long term.

\section{Discourses of performance and perfection}

I adopt Foucault's (1972) use of the term 'discourse' to explain social practices that regulate the production and spread of statements and perceptions of reality (Pringle, 2008). Thus a Foucauldian excavation of discourses within the culture of Australian swimming includes the investigation of individual experience and power occurring in relation to the discourse.

The performativity discourse [adapted from performance code], Evans, Rich \& Holroyd (2004) places importance on productivity. In the case of a sporting culture, it relates to pedagogy that focuses on and serves to regulate and monitor the output of athletes, by honing in on the specialised skills necessary for the production of the specific outputs [competitive performance]. Butler $(1990,1994)$, used the concept of performativity to specifically explore how gender identities 
are always performed, such that they can be understood as productive enactments of performances according to rules, expectations and resources available in a particular social milieu. While Butler's interest in and application of the concept of performativity (in 'Bodies that Matter') needs to be acknowledged as distinct from Ball's (2004) and Evans et al.'s (2004) use of the term, her work nevertheless similarly highlights the centrality of discourse and power relations in relation to performativity (specifically of gender). Notably, Butler (1990) draws attention to the "normalising" function of performativity and furthermore, associates performativity with "being implicated in that which one opposes" (p. 241). As we will see later in the thesis, this arguably resonates with aspects of the data relating to the three swimmers' feelings and lives as adult women.

Ball (2004) describes performativity as:

A technology; a culture and a mode of regulation, or even a system of terror that employs judgements, comparisons and displays as a means of control, attrition and change. The performances of individual subjects or organisations serve as measures of productivity or output, or displays of 'quality,' or 'moments' of promotion or inspection. They stand for, encapsulate or represent the worth, quality and value of an individual or organisation within field of judgement. (p. 143)

Within such a discourse of performativity, athletes can be viewed as malleable objects (McMahon \& Dinan-Thompson, 2008), trained and manipulated to a perceived ideal for the sake of competitive performance. Bain (1990) refers to these practices and perspectives as technocracy and states, "that within such a technocentric ideology, people are viewed as human resources where attention is focused on the development of an increasingly effective and efficient means for achieving goals" (p. 29) thus promoting competitive performance and encapsulating performativity pedagogy. Bain (1990, p. 29) uses the analogy “of the coach or choreographer treating the athlete or dancer as an instrument with which to achieve the desired performance" to describe technocracy occurring in sport. In such a case, the "participant learns to view his or her body as an instrument to be trained, manipulated and in some cases drugged for the sake of performance" (Bain, 1990, p. 29). 
Sport and particularly, elite sport, is a context and culture in which "people are viewed as human resources" (Bain, 1990, p. 29), and athletes' bodies are trained to be efficient and obedient for the sake of competitive performance. Sporting cultures can thus be seen as exemplifying a discourse of performativity. This discourse privileges the technological criteria of efficiency and effectiveness in producing measurable outcomes (Bain, 1990; Eisner, 1985) such that the technocentric ideology coalesces with meritocracy. ${ }^{11}$ Bain (1990) explains that the "political, moral, ethical and aesthetic value of goals and processes remain unexamined" (p. 29). For this reason, technocracy tends to reproduce rather than challenge existing social arrangements (Bain, 1990) permeating cultural discourse.

To fully understand the impact of culture, including how discourse is embedded, enacted and experienced in sporting culture, it is essential to recognize what culture encompasses. Sutton-Smith (1989) discusses how culture can be reflected through the idea that culture is an "information economy" (p. 47) where information is created, practiced and used thus representative of a very core of a culture. Furthermore, it is "the total socially acquired life-way or lifestyle of a group of people" (Sutton-Smith, 1989, p. 47). Sutton-Smith's metaphor of culture as an information economy aligns with Bourdieu (1988, p. 161) who links culture to body disciplines, beliefs, rituals, habitus and power. In so doing, this potentially offers some explanation to how culture is embedded and enacted through sporting culture based on the information of a cultural core.

To understand the impact and operations of discourse within a culture, I employ Tinning's (1997) interpretation, that the concept of discourse is "a recurring pattern of language or visual images about a phenomenon that portrays itself in a certain way" (p. 101). Foucault (1974) discusses the construction of discourse which occurs through a field of knowledge thus closely aligning to the concept of culture.

Like other sporting cultures where performance is imperative, swimming is a site that functions on meritocratic principles (McMahon, 2008). This is particularly the case in countries such as Australia where the sport has inherent

${ }^{11}$ Meritocracy has an extrinsic focus of achievement of competitive performance. 
ties to social, cultural and national identities. Athletes are immersed in a culture that focuses on achievement [discourse of performativity]. The importance of the body within swimming culture further resonates with the work of Bain (1990). Bain's (1990) conceptualisation of technocracy provides a pertinent underpinning for research seeking to extend insights into the pedagogy-body-culture dynamics within sporting cultures. The notion of meritocracy marries with what Bain (1990, p. 29) refers to as "the technocentric ideology where a 'swimmer body' is a valuable commodity "viewed as an instrument and object for manipulation" (Bain, 1990, p. 29) in order to achieve success. Tinning (1997) discusses the discourse of performance which occurs in sporting culture which is based around improving sporting performance. Tinning (1997) says that such a discourse is usually underpinned by science, specifically science relation to performance and the physiology of the body. The performance discourse is thus legitimated through training, measurements and tests which Vertinsky (1985) describes as "values packaged in a scientific wrapping" $(1985$, p. 73$)$. As such, ethnophysiological (McMahon \& Dinan-Thompson, in press) ideals are established in regard to an athlete body. Such values and practices are constructed and executed in support of power hierarchies resulting from specific historical events (Ellingson, 2006), such as the technocentric ideology adopted in sport and training (Bain, 1990). Subsequently, Tinning explains that the language that occurs within such a performance discourse is based on "training, workloads, thresholds, selection and supports competition usually with the less fit or able being excluded" (1997, p. 102). Thus, athlete bodies, within a performativity discourse, become subject to a wider technology based on judgement - judgement of performance, judgement of training and judgement on the way their body is shaped [perfection discourse].

Meritocratic philosophies and memes (Dawkins, 1989) [ideas, symbols and practices] potentially provide further conceptual insights into technocracy operating within a performativity discourse. Roberts (1998, p. 248) discussed the concept of memes in sporting culture, stating that they are "the cultural equivalent of genes, provide the stimulus for cultural change, a process which parallels biological evolution." Rorty (1992, p. 581) further explains, "a meme is the cultural counterpart of the gene." Subsequently, Roberts (1998) identified memes as the core of a sporting culture's logic which can include overarching practices, 
logic and rationality. Memes are thus, important to cultural transmission and inherent in and through lived experiences.

In my previous research (see McMahon \& Dinan-Thompson, in press), ideologies relating to performance and perfection occurring through the regulation of a swimmer's body [my swimmer body] in Australian swimming culture were researched. The research revealed how the discourses of performance and perfection became normalised through ethnophysiological practices and were emblematic of the Australian elite swimming culture. The ethnophysiological practices described in the stories could be considered as a psychopathological crystallisation of culture as my body was constrained and trained in docility and obedience (Bordo, 1993). In the context of the regulatory practices, the regulators including myself strived to enhance my body's utility, productivity and performance through regulation, which "increased its manipulability" (Featherstone, 1991, p. 250). The body was made "pliable and amenable" (Featherstone 1991, p. 250) to regulatory norms that operated to increase my swimmer performance. However, the swimmer body was not simply a technocentric object in the elite culture that could be manipulated for performance, but "a sentient being whose primary relation to the culture should be understood in terms of this meaningful sentience" (Crossley, 1996, p. 47). In response to the regulation and lack of self-determination in regard to my body, I became a monomaniac and engaged with a risky self-regulatory behaviour in an act of conformation and regaining of bodily control. Howe (2004) states: "such risk is a product distinctive of that particular sporting culture where cultural capital is accrued by those who are willing to risk their health and well-being for a better performance” (p. 125). My behaviour could be seen as my response, “acquired through a social process of becoming” (Monoghan, 1997, p. 284) within the elite swimming culture. Regulatory practices were exerted through power and disciplinary practices (coach, mother and self). Foucault (1980a) states: "power is neither given, nor exchanged, nor recovered but exercised - it exists only in action" (p. 89). Thus, regulation of my body was incurred through action and my bodily behaviour, as a direct result of my immersion in the elite swimming culture, my desire for high performance, and for social acceptance. This research revealed parts of my individual experiences, and in turn, the complexities of bodywork and sociological issues in regard to performance and perfection 
occurring within the elite Australian swimming culture. It pointed to the need for more research into the socialisation of the swimmer body within the Australian swimming culture and the effects that action; structures of power and control occurring through regulatory practices.

Previous research [see McMahon and Dinan-Thompson, 2008] drawn from my (McMahon) honours' work where I provided an autoethnographic account of my own experiences as an elite swimmer positioned swimming as a social practice and detailed the deep-rooted technocentric and meritocratic ideologies prevalent in the Australian Swimming culture. Technocentric ideologies were found to be promoted through the concepts of productivity, efficiency and performance and to thereby create Australian Swimming 'body norms' which were (re)inscribed through regulatory practices over an extensive period of time. My (McMahon) stories revealed how my body was a "site of explicit exposure" (Pillow, 1997, p. 349) where it became a malleable object, manipulated by myself and other social regulators to a perceived ideal for the sake of performance. Conversely, my stories also demonstrated that the body may not be as malleable as the Australian Swimming culture would like. This work illustrated that I experienced feelings of distress about my body and yet at the same time, contributed to the reproduction and reinforcement of a 'swimmer body.' My experiences revealed a vulnerable self that had been moved by, and moved through, complied and resisted cultural interpretations (adapted from Ellis \& Bochner, 2000) evincing variations in embodiment. It was concluded that more research was needed into the exploration of the regulatory practices in the Australian Swimming culture and the impact they have on the body and sense of self.

Research by Jones, Glintmeyer and McKenzie (2005) provides us with a further insight into the effects of a culture of slenderness, specifically in the sport of swimming. Using interpretive biography, Jones et al. (2005) explored the experiences of a former elite swimmer and her compliance within a swimming culture of slenderness and norms. Their research also examined the coach's role within that culture. Jones et al. (2005) revealed that the participant believed her swimmer experiences led her to develop an eating disorder. However, the research does not excavate the relationship that the swimmer has with her body in the 
present day; that is, post swimming career. Thus, the research predominantly focuses on her swimmer experiences. Jones et al.'s (2005) findings showed that due to a strong athletic identity, the elite swimmer developed a vulnerable sense of self, which, when disrupted, contributed to the development of an eating disorder. Using Foucault's (1977) concepts of disciplinary power including surveillance and normalization as well as investigating the differential power relationships of the coach/athlete relationship as part of their analysis, they explained how the discourse 'fed the disorder' through ongoing surveillance and disciplining of the self. Their work arguably pointed to the value of a Foucauldian framework in work that seeks to analyse sporting discourses, specifically power and knowledge occurring in such a discourse.

Purdy, Potrac and Jones (2008) conducted research into sports coaching, more specifically the dynamics that exist between a coach and athlete using autoethnography. Purdy, the primary author of the research, drew upon her own experiences as an elite rower to excavate the complexity that occurs between a coach and an athlete relationship. Purdy et al.'s (2008) research, although conducted within the culture of a different sport [rowing], is particularly pertinent to other sporting cultures as it articulates the various and constantly changing measures of athlete compliance, cooperation and resistance to a coaching programme. More specifically, the key themes identified in their analysis including issues of power, consent and resistance will also be relevant to this research into the Australian swimming culture. Methodologically speaking, autoethnography was purposively chosen in the research conducted by Purdy et al. (2008) due to its capacity to construct the "personal complete with micro-power issues and consequences, within a public macro social milieu" (Purdy et al., 2008, p. 333; see also Clandinin \& Connelly, 1991). For the authors autoethnography represented "a valuable means for investigating the realities and problems of human interaction, which is an essential feature of sports coaching" (Purdy et al., 2008, p. 333). Subsequently, this study also employs autoethnography in conjunction with narrative ethnography by drawing on the experiences of three participants (the primary author and two others) as detailed in the introductory chapter. Dissimilarly to Purdy et al.'s study, this research provides a longitudinal aspect by investigating how the coach-athlete relationship can affect, if at all, the athlete in the long term. 
Research into surveillance and conformity in 'competitive youth swimming in the United Kingdom' (UK) was conducted by Lang (2010) utilising an ethnographic approach. Using observations and semi-structured interviews, Lang identified the disciplinary mechanism of surveillance at work in competitive youth swimming. More specifically, her research excavated the ways in which the bodies of swimmers and their coaches are subject to and apply controlling mechanisms to produce embodied conformity to normative behaviour and obedient, docile bodies (Foucault, 1977). Lang's application of Foucault's panopticon to the UK swimming culture, more specifically the swimming pool [training environment], is particularly pertinent to this study and will be applied accordingly. Her research revealed that both coaches and athletes were under constant surveillance which consequently led them to internalise the panoptic gaze (Lang, 2010). Other Foucauldian themes used by Lang in her research include; disciplinary power, surveillance, technologies of the self and conformity which will be applied accordingly to this thesis specifically through the interrogation of the Australian swimming culture. This thesis builds on Lang's work by using a longitudinal approach enabling the long term effects of body pedagogies to be explored.

Lee and Macdonald (2010) utilised a longitudinal approach to their research to explore how the discourse of healthism is perpetuated through the experience of a group of young rural women, specifically in the context of physical education (PE) in a school. Their research is particularly relevant to this thesis which also explores the effects of discourse, specifically as they also examined the "durability" (Lee \& Macdonald, 2010) of the discourse using a longitudinal approach. Using in-depth interviews and visual collection methods, their qualitative research sought to understand how the healthism discourse was reproduced through social structures and how it perpetuated such structures creating implications for health. Using Bourdieu (1991), social reproduction, the linguistic market [Bourdieu's view that discourse is central to understanding social relations] and the habitus of the young women was examined to explore the dominance and stability of the discourse.

Perfection discourse [adapted from perfection code, Evans, Rich \& Holroyd 2004] closely aligns with the discourse of performativity but specifically relates to 
pedagogy and social interactions based on the ideology that the body is imperfect and in need of being changed. When applied to a sporting culture, the athlete body would be viewed as unfinished with the intention of amelioration [make or become better] occurring. The discourse of body perfection can be described as "structures of meaning defining what the body [in size, shape, predisposition and demeanour] is and ought to be; and how, for those who do not meet these ideals, there is treatment, repair and restoration" (Rich \& Evans, 2007, p. 44). Athlete bodies are managed, regulated, measured and compared against standards within the elite sport discourse and thus their results are subject to a wider technology which judges (Rich \& Evans, 2007) how a body measures up these standards. For those bodies that do not meet cultural standards, Evans, Rich \& Holroyd's (2004) notion of the threatened body [body shape threatened through neglect] becomes pertinent.

Perfection pedagogy derives from the 'pedagogical palette' (Bernstein, 1975; Evans, Rich \& Holroyd, 2004) with specific attention placed on the regulation of the body. Evans et al. (2008, p. 98) highlight that "all pedagogical relations are power relations; there is no instruction without regulation; no pedagogy is divorced from control" (Evans et al., 2008, p. 98). The logic of performativity and perfection when applied to a sporting culture, is not only confined to the training discourse but rather, can be seen to extend "more explicitly onto and through young people's bodies via manifest aspects of corporeal perfection via what we have referred to as body pedagogies" (Rich \& Evans, 2007).

In seeking to extend this understanding of pedagogy, the work of Foucault (1977) is also highly pertinent as it connects the body, power and culture. The 'pedagogical devices' (Bernstein, 1996) relating to performance and perfection that are carried out in a sporting culture are representative of a specific site where particular techniques of power become concentrated and are brought to bear on individuals, the athletes in systematic ways. Foucault views the notion of power as the constitutive forces that shape and create rather than destroying or oppressing (Garrett, 2004), encapsulating the very essence of the performativity and perfection discourses that pervade elite sporting culture. Hence, Foucault's notion 
of the body is that of a surface, acted upon by culture, inscribed by power and mediated in discourse (Garrett, 2004).

Foucault's concepts on modern disciplinary power are thus salient to this thesis, specifically his thinking in regard to mechanisms of power, where bodies embody conformity to normative behaviour and as such become obedient and docile (Foucault, 1977). Thus, power is bound in the production of knowledge [body pedagogies] and has the ability to define what is accepted as 'normal' (Lang, 2010). As the body is central to sport and physical activity, other scholars have found Foucault particularly relevant and utilised his concepts of disciplinary power (Hall, 1996; Theberge, 1987). Foucault (1991, pp. 137-138) explains disciplinary power as:

how one may have a hold over others' bodies, not only so that they may do what one wishes, but so that they may operate as one wishes, with the techniques, the speed and the efficiency that one determines. Thus discipline produces subjected and practised bodies, 'docile' bodies.

Disciplinary power, portrayed through acts of control over the body, is enacted with the intention of producing docile bodies which are "subjected, used, transformed and improved" (Foucault, 1978, p. 136) exemplary of a performance and perfection discourse. Ultimately, when applying Foucault's interpretation of power to a sporting culture, it can be enacted through pedagogy, subsequently focusing on the athlete as both an object and a subject of power relations. Foucault viewed "power as everywhere, not because it embraces everything, but because it comes from everywhere. Power is not an institution, nor a structure, nor a possession. It is the name we give to a complex strategic situation in a particular society" (Foucault, 1979, p. 93).

Foucault's work on disciplinary power includes the organisation and regulation of time, space and movement. When applying Foucault's conceptualisation of disciplinary power to this thesis, more specifically a sporting culture, it occurs when it permeates the athletes' lives by training, shaping and impressing their athlete bodies with habitual gestures, procedures and values. Like in other disciplines, sporting cultures involve both the discipline as a body of 
knowledge and discipline as control, "such that the body of knowledge that constitutes the discipline of high-performance sport is knowledge of the technologies that discipline or control athletic bodies" (Shogan, 1999, pp. 9-10). Shogan (1999) explains how "disciplinary power is exercised both through the subject matter of a discipline conveyed through discourse and at the level of the body controlled through technology" (1999, p. 10). In the sections that follow, I have organised the assemblage of literature relating to disciplinary power (Foucault, 1977) pertinent to this thesis, using sub-headings of panopticism/discipline, surveillance, docile bodies and normalization with the intention of assisting in explaining the operations of modern power.

Foucault expressed that power in its capillary form of existence, "reaches into the very grain of individuals, touches their bodies and inserts itself into their action and attitudes, their discourses, learning processes and everyday lives" (Foucault, 1977, p. 39).

Foucault further explains that:

discipline may be identified neither with an institution nor with any apparatus; it is a type of power, a modality for its existence, comprising a whole set of instruments, techniques, procedures, levels of application, targets; it is a 'physics' or an 'anatomy' of power, a technology. (1977, p. 215)

For Foucault (1977), the body is a subject and object of technologies of power, occurring through micro-practices, which when applied to a sporting culture can be exemplified thorough the creation of obedient athletes.

\section{The aquatic back drop - the Panopticon}

Foucault used the metaphor - panopticon to articulate the operations of power and surveillance occurring in contemporary society. When applied to this thesis, more specifically a sporting culture, the metaphor of the panopticon offers a useful way of understanding the power relationships occurring between an athlete and a coach and how social control can be administered without coercion. Other authors 
to apply panopticism to research surrounding sociology of sport include Andrews (2000), Chapman (1997), Cole (1993), Duncan (1994), Markula (1995), Rail and Harvey (1995), Rinehart (1998), Shogan (1999) and more recently specifically to swimming, Lang (2010).

To illustrate the power of the panopticon, Foucault's (1979) utilises a design of a prison produced by Jeremy Bentham. It was an architectural model of modern disciplinary power, with focus placed on an annular building within the confines of the periphery. At the centre of the annular building was a tower with a supervisor placed in it, situated there to observe the [prisoners] below. Thus, the prisoners were the potential targets of authority's gaze with the intention that they would regulate their behaviour, conforming to a set of behaviours that were prescribed and thus expected of them to avoid potential punishment. Although the prison was never actually built the idea was used as a model for numerous institutions. In contemporary society, we can apply panopticism to operations of surveillance. "On a broader scale, panopticism refers to a political anatomy where the mechanisms of surveillance and discipline are no longer locked within specific buildings or institutions but function in a diffused, multiple, polyvalent way throughout the whole social body" (Markula \& Pringle, 2006, p. 43; see also Foucault, 1991, pp. 208-209).

For the purpose of this thesis, Foucault's (1979) concept of the panopticon is applied to the Australian swimming culture with the swimming pool itself doubling as the central quarters, with the coach and/or team managers who stand on the pool concourse illuminating that of a supervisor [guard], governing over the swimmer athletes in the water below. "The swimming pool is the diagram of a power that acts by means of general visibility" (Lang, 2010, p. 31; see also Foucault, 1991, p. 190) which, in turn promotes the panoptic gaze of the coaches and team managers. The panoptic gaze, in so doing allows those from above [pool concourse level] as well as the panoptic gaze of athletes on each other to judge the degree to which the training regime has been adhered to (Johns \& Johns, 2000).

For Foucault, the panopticon integrated power and knowledge. In the case of this thesis, the panopticon [swimming pool] and the culture it is associated with exerts control over the swimmer 'body' through the control of space and through the technology of discipline. Thus, within the periphery of the aquatic facility [the 
panopticon], the swimmers' bodies are made productive and observable (Foucault, 1977, p. 44) thus becoming subjects and objects of the discourses of performance and perfection of the Australian swimming culture. The panoptican [pool] is thus a superbly efficient mechanism for social control as the "omnipresent gaze of authority subsequently disciplines the subjects [the swimmers] to survey their own behaviours in a manner that renders them docile: they become their own supervisors" (Markula \& Pringle, 2006, p. 43).

\section{Surveillance (gaze)}

The panopticon could also be construed as a powerful symbol of control through the internalisation and normalisation of control mechanisms. Using Foucault's interpretation of the panopticon, another central feature of his work was the way in which the gaze constructs people [in this case - the athletes] as both subjects and objects of power and knowledge. Discipline, for Foucault (1979) is different from force or violence because it is a way of controlling the operations and positions of the body. Thus, it was in Discipline and Punishment (1979), Foucault observed that control no longer required physical domination through the use of firearms and material constraints over the body, but could be achieved through isolation and "a gaze - an inspecting gaze, a gaze which each individual under its weight will end by interiorising to the point that he is his own overseer with each individual thus exercising this surveillance over, and against herself [sic]" (Foucault, 1980a, p. 155). Subsequently, more significant than the surveillance by the supervisor [coach, team managers] discussed by Foucault (1979), is the development of self-surveillance by the prisoners [swimmers] themselves, and the acceptance of the normality of self-surveillance. Subsequently, the external application of surveillance eventually becomes unnecessary as the swimmers [prisoner] "inscribe in themselves [sic] the power relation in which they [sic] simultaneously play both roles, they [sic] become the principal of their [sic] own subjection" (Foucault, 1977, p. 202). Surveillance thus becomes a technique for self-control or self-discipline. Such a gaze, Foucault (1980b) referred to as a "voyeur's gaze - an inspecting gaze which each individual interiorize until they begin exercising surveillance over themselves" (Foucault, 1980b, p. 155). In so doing, the gaze is pervasive and powerful and the fact that it is silent, makes it 
even more powerful (Bain, 1990).Thus, the gaze is a grand formula - a superb power that is "exercised continuously and for what turns [sic] out to be minimal cost" (Foucault, 1980a, p. 155). Surveillance thus becomes a practice of selfcontrol or self-discipline as the swimmer athletes become the subject of their own surveillance. In so doing, they reflect the gaze back onto their own athlete bodies, further increasing the power of the gaze (Lang, 2010; Tsang, 2000) and in so doing technologies of the self occur. Germov and Williams (1999, p. 126) refer to this self-surveillance as the "body panopticon" which can be referred to the "constant monitoring of their own bodies and others" (p. 126) which in Foucault's (1979) words are a power situation where they are themselves are the "bearers of their own self-surveillance" (p. 201). Foucault uses the concept, "technologies of the self' to explain the ways individuals engage in practices which they "effect by their own means, or with the help of others, a certain number of operations on their own bodies and souls, thoughts, conduct, and way of being, so as to transform themselves" (Foucault, 1997, p. 225). This modality of power is capable of enforcing norms even when there may not be anyone observing one's behaviour. Germov and Williams (1999, p. 125) conceptualise this as the "body police" that they describe as the "internalisation of the cultural imperatives of the thin ideal where one is encouraged to modify and monitor themselves and others in a never-ending process of body surveillance to conform to the culturally accepted body".

\section{Docile bodies}

Docile bodies are productive bodies and are created as a result of disciplinary power (Foucault, 1979):

Foucault used the notion of docility to demonstrate how bodies become manipulable and effective means for discipline. When docile, the body becomes useful as it can be moulded [discourses of performance and perfection] as a vehicle for the technologies of domination. (Markula \& Pringle, 2006, p. 74) 
The idea of docility evolved from Foucault's work in Discipline and Punish (1978), where he argued that individuals are under constant surveillance and regulation in ways that are often subtle and thereby seemingly invisible, leading to normalization and acceptance of such systems.

Foucault focused on the body specifically as the site of regulation, or more specifically "as object and target of power" (Foucault, 1991, p. 136). The notion of 'docility' was employed by Foucault and thus will be applied to this research, to illustrate how individuals are subjected to institutional regulation (Foucault, 1991, p. 136). Docility is achieved through discipline. For the purpose of this thesis, discipline is multi-dimensional as it includes 'discipline of the self' [technologies of self] as well as 'discipline imposed by others' by the virtue of power relations. Discipline is different from force or violence because it is a way of controlling the operations and positions of the body. For Foucault, docility achieved obedience and compliance, whilst utility assisted productivity. The imposed 'docility-utility' of the body supposed that as it became more obedient it becomes more useful.

Johns and Johns (2000) revealed 'docility-utility' occurring in women's gymnastics. In so doing, their findings can be applied to this thesis. As I discuss further below, there is an important dimension of normalization reflected in the practices and expectations seen in Australian swimming culture. Athletes are expected to complete sessions twice daily with each training session broken down into parts which include, warm up of stretching designed to increase muscle flexibility followed by approximately two kilometres of swimming where coaches prepare the swimmer bodies for the main set and session ahead. After the warm up is completed, the main set is executed which can be made up of aerobic training or anaerobic training. This training is devised for a purpose, to increase the swimmer body in speed and/or endurance and can last between two kilometres and four kilometres in length. Once the main set is completed, the coach will finish the session with kick and a warm down of approximately one to two kilometres. The warm down phase is conducted to prepare the swimmer body for the next session that day so it can be completed effectively. Thus, each training session is broken down into elements, articulations are defined, each set is assigned a direction and duration and the order of succession is prescribed. Time 
penetrates the body and with it, the meticulous controls of power occur (Foucault, 1991; Johns and Johns, 2000) and the discourse of performance is engaged with.

\section{Normalisation}

In Foucauldian terms, normalisation refers to social processes through which ideas and actions come to be seen as normal. Thus, when applied to this thesis, normalisation refers to the training and bodily practices that athletes undergo on a regular basis based on the idea of enhancing competitive performance [discourses of performativity and perfection] which have become normal. These specific bodily practices can "determine the conduct of individuals [swimmers] and submit them to certain ends of domination" (Foucault, 1988, p. 18). For Foucault (1977), normalization was one of many tactics relating to power where the exertion of maximum social control could be achieved with the minimum expenditure of force, which Foucault explained to be disciplinary power. Normalisation can also include social processes by which new ways of thinking, working and organizing become routinely incorporated in everyday work and practices.

Foucault (1977) saw social organization and regulation occurring most effectively through practices of normalization. For Foucault (1977), normalising power involved the dimensions of physical and biological discourses and how these are inserted on the human body. In sociological studies of sport and exercise, a number of researchers have undertaken Foucault's thesis by examining how sport practices "act as technologies of domination that encourage individuals into a discursive web of normalising practices" (Markula, 2003, p. 88). These scholars include; Cole (1993), Halas and Hanson (2001), Hargreaves (1986), Heikkala (1993) and Markula (1995, 2001). Normalization both constrains and enables the swimmer body and in so doing affects their athletic capacity to make and act on judgments that are meaningfully their own. 


\section{Situating the [swimmer] body within a culture}

The body has gained attention as a place "from which to theorize, analyse, practice, and critically reconsider the construction and reproduction of knowledge, power, class and culture" (Pillow, 1997, p. 349). Recent work (Amour, 1999; Bordo, 1989, 1992; Frost, 2001; Grosz, 1995; Kirk, 1993) on the body suggests that bodies "are both inscribed with and vehicles of culture" (Garrett, 2004, p. 140). Culture circulates through all of us, for our self is a social phenomenon, all identity is relational, and our individual subjective experience is a result of the world [culture] for which we inhabit (Sparkes, 2004). For Bordo (1989) who applied Foucauldian concepts to the body and culture, the body - what we eat, how we dress, the daily rituals through which we attend to the body is a median of culture. "Our bodies, no less than anything else that is human, are constituted by culture” (Bordo, 1996, p. 392). For Grosz, (1995, p. 35), "bodies speak without necessarily talking, because they are coded as signs. They speak social codes. They become intextuated, narrativitized, simultaneously; social codes, laws, norms and ideas become incarnated." Thus, normalised bodily practices with a sporting culture are "made body" and "converted into automatic, habitual activity" (Bourdieu, 1977, p. 94). Arthurs and Grimshaw (1999) say that, "the body is itself the subject of constant social inscription; it is discursively constructed and written on by innumerable forms of social discipline" (p. 7).

When discussing the effects of cultural incarnation and social reproduction, the concept of habitus (Bourdieu, 1984) thus becomes relevant. A number of scholars of bodily practices (Crossley, 2004, 2006a, 2006b, 2007; Wainwright \& Turner, 2004; Wainright, Williams \& Turner, 2007) and embodiment have drawn upon Bourdieu's (1984) notion of habitus which can be described as a set of dispositions (lasting, acquired schemes of thought and action) which generate practices and perceptions. The individual subsequently develops these dispositions in response to the objective conditions [culture] it encounters. Habitus, thus is an outcome of social practice and explains the embodied dispositions of a person (Evans, 2004). Subsequently, Bourdieu $(1979,1986)$ was one of the first social scholars to consider sport as a serious sociological issue. Bourdieu's notion of habitus, in essence for Crossley (2001, p. 106) "illuminates the circular process whereby practices are incorporated within the body, only then 
to be regenerated through the embodied work and competence of the body." Crossley's interpretation of Bourdieu's habitus encapsulates the very essence of body pedagogies and embodiment - another central aspect of habitus. Bourdieu's notion of practice, disposition and habitus (Bourdieu, 1984, 1990) can describe the concept of embodiment. Embodiment is the lived experience of a body, "a textual surface upon which a person's life is inscribed" (Sparkes, 2004, p. 157) and it is in the sense very close to the notion of practice (Turner, 2000) - to form into a body; to invest with a body and to collect into a body. Embodiment is the ensemble of corporeal [tangible; physical; of or pertaining to the body; bodily; corporal] practices which produce and give a body its place in everyday life. Embodiment locates or places particular bodies within a social habitus. Jackson (1990, p. 48) explains the notion of embodiment:

Even though my body seems the most private and hidden part of me, I carry my life history in my body, almost like the age rings of a sawn tree trunk reveal the process through time. My personal history of social practices and relationships is physically embodied in the customary ways I hold my body, imagine its size and shape, and in its daily movements and interactions.

Tinning, McCuaig and lisahunter (2006, p. 125) described embodiment as learning through one's body, "as a body, for the body, about the body and to the body, yet going beyond the body as an object or thing." They further state, "being associated with the body both in how one's dispositions are played out through the body and how one's body is constituted or understood by others." In so doing, we experience our bodies "in ways that provide constant reminders that being human is to be embodied" (Kirk, 2002, p. 79).

\section{Conclusion}

With a few exceptions (Beckett, 2004; Davis, 2004; Evans, Rich \& Holroyd, 2004, 2008; McWilliam, 1999; Shilling, 2004; Wright \& Harwood, 2007; Zembylas, 2007), few have made the affective and embodied dimensions of pedagogy a primary concern (Evans et al., 2008, p. 110). Research and critique 
that addresses the exposure and effect of body pedagogies including the socialisation of the swimmer body, the effects that memes, action, structures of power, occurring through practice, have on the body and self both in the short term and also long term, specifically in the context of the Australian swimming culture is still largely lacking in the social sciences. In this respect, little has changed since Theberge (1991, p. 148) commented that work in the sociology of sport "has been largely devoid of consideration of the body and of embodiment." Subsequently, in an attempt to address this gap in scholarship, this thesis has drawn upon a somewhat eclectic body of literature to provide an appropriate theoretical lens to be applied to the stories of experience pertaining to the Australian swimming context. This thesis draws upon some of Foucault's main concepts such as the gaze, surveillance, normalisation and discipline and employs them to help explicate the interpretation of the stories [primary data] told. 
CHAPTER THREE 


\section{Narrative ethnography and autoethnography: Empowering athletes and their bodies in and through research}

\section{Introduction}

In this chapter, I describe how narrative research utilising an ethnographic framework and autoethnography evolved as optimal methodologies for this doctoral research project. The term 'narrative ethnography' (also used by Gubrium \& Holstein, 1999; Tedlock, 1991) is used throughout this chapter to refer to narrative research which is shaped and informed by ethnography and undertaken within an ethnographic framework and design. The purpose of the chapter is to outline why and how I came to employ narrative ethnography and autoethnography with specific agendas, relating to both the participants and intended audiences for this research, and to critique the strengths and weaknesses of the methodology specifically in relation to one of the prime methodological aims; the empowerment of participants in the research process.

The title of this chapter signals that emancipation [with the potential of achieving] was a key concept shaping what methodological framework was employed for this thesis. Laurel Richardson's (1995) thesis that how we are expected to write affects what we can write about, also guided me in relation to my methodology. Narrative ethnography and autoethnography provided myself and the other two participants with the appropriate space to reveal our knowing and detail our lived experiences. Once I had gained ethics approval (see Appendixes A and B), supplied participants with an information sheet (see Appendix C) on the study, and achieved the necessary consent from the participants (see Appendix D), I had a telephone conversation with one of the participants. This conversation occurred when this research was in its infancy and led me to critically reflect on my approach and position in the research. Specifically, I was prompted to consider ways in which I could enhance the collaborative dimension of my work and ensure that the participants' best interests were considered and their participation could ultimately be rendered meaningful. 


\section{A telephone conversation from January 2008}

Jenny: "Good news! I got ethics through for my PhD last week so we will be starting to collect data from you soon. I will explain more about what we are going to do when you are back from holidays."

Participant: "It is ok; I can talk now for a while. I am just sitting here in the hotel room."

Jenny: "Well, I think that we are going to present your stories in the form of unsent letters which means that you would write a story of your experience in a letter format."

Participant: "What do you mean? Like who would I write a letter to?"

Jenny: "There could be a number of people. Think about who you really would like to write a letter to in your swimming career. It could be your Mother, coach, teammate or school friend? It is totally up to you."

Participant: "I think that I would like to write a letter to the Australian coaches who were on the Olympic swimming team with me."

Jenny: "We are also going to look into stuff on your coaching practices, what you do and how you do it. You will have to write something about that as well. I will have to get your athletes to verify what you have written - to validate what you are saying."

Participant: "What do you mean? Don't you believe what I am going to tell you will be true?

Jenny: "Of course. It is not that. It is kind of what I am expected to do in academia."

Reflecting on this telephone conversation, I realised that as a researcher, I was in a position of relative power, influence and affluence and needed to strive to be more collaborative in my approach. The participant had little voice in the conversation and my intentions for the research. The more I thought about it, the more uncomfortable I became, realising that I was being exploitive. My intention was that the participant's stories would form the primary data of the thesis and 
represent the very essence of the research. Their voices, my findings, were what could help me make a difference. I realised that I had to alter my approach and implement a framework that would give the research participants the opportunity to achieve emancipation in the research, with the intention of empowering them through their participation.

My previous experiences as an elite swimmer (see McMahon \& DinanThompson, 2008) provided me with further impetus to seek out a methodology that would address four important considerations that I regarded as paramount in this research. The first addressed the participants and their voice in the telling of their stories (the primary data). Specifically, the research sought to foreground the swimmers' voice and the body's voice in the research. I wanted the swimmers' voice and their body's voice to be employed as the primary data of the research, with the research process seeking to specifically utilise the swimmers' strong connection with their bodies, their abilities to 'listen to their body' and awareness of 'how it was feeling,' where it was sore, whether it could be pushed further. A related concern was to ensure that power differentials experienced within the Australian swimming culture would not be replicated through the research process. My methodology needed to enable the three participants to have what I see as 'meaningful participation' in the research, positioning them in a genuine (rather than tokenistic) collaborative relationship with the researchers, and thus providing a distinct contrast to the inequitable power-relationships that I (McMahon \& Dinan-Thompson, 2008) described with my immersion in the Australian elite swimming culture.

Consideration was also given to audience, with the intention that the reader would be able to take on and read from the positions and perspectives of the participants, vicariously sharing in their experiences as three adolescent elite swimmers and subsequently, as 30-40 year old women. The appropriate methodology needed to allow the stories of experience - the primary data - to be presented in such a way that would allow all readers from various backgrounds the opportunity to access and vicariously share in the participants' lived experiences.

Finally, I wanted the participants to gain some purpose from their involvement in the research and recognised that there may be emancipatory 
potential in this work. This consideration emerged from an initial telephone conversation with one of the participants. Specifically, the act of participation could provide an opportunity for the participants to seek some clarity, gain new insight and achieve 'order' in relation to their swimmer experiences. In my view, the methodology should seek to facilitate and not deny nor overlook that emancipatory potential. For Hickey and Austin (2007), autoethnography opens possibilities for participants to be critically reflexive wherein senses of their self and agency might come to be understood in terms of the social processes that mediated their lived experience. I sought to pursue similar potential in using narrative ethnography in combination with autoethnography.

Below I describe how I developed my methodology to address each of the key considerations outlined above. While the considerations provide the focus and structure for this chapter, inevitably, some of the points raised are pertinent to more than one of the considerations. In the concluding section, I focus specifically on the issue of empowerment occurring in and through the research, considering the ways and extent to which the chosen methodology empowered the three participants.

\section{Selecting and shaping the research methodology with specific agendas}

As indicated above my choice and development of the research methodology for this study related primarily to four considerations:

- to foreground the swimmers' voice and the body's voice in the research;

- to achieve and maintain highly collaborative relations throughout the research process;

- to enable the reader to take on and read from the positions and perspectives of the participants, vicariously sharing in their experiences as three adolescent elite swimmers and as 30-40 year old women; and

- to ensure that the research process had purpose for the participants, with specific recognition that there may be emancipatory potential in this work. 
In discussing the detail of the methodology, I focus specifically on these points, highlighting the inter-related nature of my concerns and elements of tension between them.

\section{Employing narrative ethnography and autoethnography}

Simplistically, narrative can be described as a qualitative research method that refers to any written or verbal representation (Polkinghorne, 1988; Riessman, 1993). For me, narrative was the subject of inquiry, the method of interpretation, influencing how the text was created thus impacting the research experience overall while allowing the lived experience/s of the swimmers to be presented. In this research, I use the term 'narrative' in the way Sarbin (1986, p. 3) uses it as "the organizational scheme I use to make meaning of experience in a story form." Specifically, narrative ethnography and autoethnography allowed the stories, voices, and experiences of myself and the other two participants within the culture of Australian swimming to be employed as the primary data - taking the centre stage, as well as enabling me to address my three other key methodological considerations [power, audience, emancipatory emphasis]. For Ellis and Flaherty (1992, p. 170), "the relationship between lived experience and narrative is significant. The narration mediates the experience into a social form." As a researcher-participant, autoethnography has enabled me to write in the first person, connecting my personal experiences to the cultural (Ellis \& Bochner, 2000, p. 739), writing about (my)self refracting elite sporting culture.

The sections that follow examine in detail the particular ways in which I employed narrative ethnography and autoethnography. I highlight how these methodologies can be employed in many ways, with varying effects from researcher, participant and audience perspectives, and that methodological details are crucial in relation to whether or not considerations such as those I fore grounded would be met. 


\section{Constructing, re-constructing and (re)presenting stories ${ }^{12}$}

The way in which stories would be generated in the research and be presented as research data, were key issues relevant to all four of my considerations. I was acutely aware of the prospective tensions inherent in seeking to retain authenticity, accord authority to the participants in the research process, 'open the door' in relation to the emancipatory potential of participant involvement in the research, and simultaneously, generate text that would deeply engage readers with the issues I was pursuing. As researcher-participant, I played the central role in addressing and mediating these issues and prospective tensions.

Cortazzi (1993) identified that narrative enables us to "open [sic] a window on the mind, or, if I am analysing narratives of a specific group of tellers [as is occurring in this study], as opening a window on their culture" (p. 2). Through the telling of the swimmers' stories, the intention was to reveal the core of a very specific culture in which the participants were immersed, connecting their world within the culture of Australian swimming to the outer world - the readers' world. 'Opening a window' on the swimmers' minds, their lived and embodied experiences both as adolescents and as adult women, in a manner that would accord them both respect and authority in the research process, would not be easy. As an emic (an insider of the culture), I would be able to provide valuable insight into the Australian swimming culture giving depth to my role as both a researcher and participant, and hopefully, enhancing the richness of the data.

Initially, an "activity based task" (Rich, Holroyd \& Evans, 2004, p. 174) was given to each participant where they were asked to construct their stories of experience. Using memory writing (Lupton, 1996), I asked each participant to recall and write about their adolescent swimming experiences within the Australian swimming culture using a narrative representation of their choice (i.e., letter writing, poetry, stories etc). Practices that we experienced within the Australian swimming culture provided the focus for each piece of writing. Each

\footnotetext{
${ }^{12}$ Following Polkinghorne (1988), the term 'story' and 'vignette' are used equivocally to 'narrative' throughout the research. 'Story' refers to "the process of making a story, to the cognitive scheme of the story, or to the result of the process - also called stories" (p. 13), thus encompassing the very essence of narrative.
} 
participant chose story writing in these initial stages to detail their lived experiences.

From these narrative representations, I highlighted narrative threads, tensions and themes (Clandinin \& Connelly, 2000) in the stories, relating the data to the underpinning theoretical framework developed from Foucault's (1977) work, and more recent work in the field of health and physical education that has pursued a socio-critical perspective and specifically, focused on the body. Thus Evans et al.'s (2008) work on the body pedagogies together with others including, Bain (1990), Bordo (1989, 1992, 1993, 1997), Jones et al., (2002) and Jones et al., (2005) was also utilised. Prominent themes were identified by highlighting points in the data and labelling them using the relevant literature from the theoretical framework (see Chapter 2). An example of this process is detailed below where some extracts from the data with the subsequent label/s that were identified from them.

Carly's coach (to Carly): "If you put on weight you will get your periods, then your body will change and you will swim slow" [Slim to win/discourses of perfection and performance].

Team Manager (to me): “Jenny, we are concerned with your weight increases. You need to think about whether you still want a place on the team because your weight is indicating that you are not remaining focused and committed. Your skin fold readings show us that you are losing fitness. We will be interested to see how you go in your skin fold measurements tomorrow? " [Slim to win/discourses of perfection and performance/disciplinary power]

A number of themes were identified included; technocentric ideologies, slim to win ideologies, differential power relationships, self-surveillance, disciplinary power, discourses of performance and perfection relating to the body and performance, and meritocracy [see chapter five for further discussion of themes] all of which are occurring in and through body pedagogies.

I then met with the participants face to face in a location of their choice, specifically somewhere where the participants felt comfortable, to extend the data. Using the key themes as a prompt and topic of discussion, I sought further 
explanation and expansion from each of the participants in relation to aspects of their original stories. In this phase of the research, to ensure that the process was meaningful from a participant perspective, a point [key theme] was introduced to each participant in a way that related to the discourse in which they had been immersed and would be able to relate to. For example, the theme of technocracy was addressed by asking the participants about practices and incidents that they could recall which related to monitoring of their body linked to a focus on performance (and specifically, attainment or maintenance of specific times for events). The question relating to a key theme was used as a prompt, purposely encouraging the participant to help shape the direction of the discussion, with the emphasis that it would be their stories that would determine that direction. Visual prompts such as pictures and video footage from the time the swimmers were training and competing were also used at this stage to prompt memories, assisting the participants to visualize their presence back in the scene (Australian swimming culture) both physically and emotionally.

The emphasis of emancipation influenced how the interviews were conducted. Thus, the length and structure of interviews was flexible, highly dependent upon participant response. Fontana and Frey (2000) view such flexibility as an attempt to understand the complex behaviour of members of society without imposing or limiting discussion and thus limiting the field of inquiry. Ultimately, interviews spanned several days, with the number and duration of meetings determined by how the participants were feeling emotionally and physically and whether they wanted to continue the discussions or not. As an autoethnographer who had previously recalled her own adolescent experiences as a member of the Australian swimming team, I was mindful that the recalling of such experiences may be painful. To provide the appropriate support to the participants throughout the research process, I organised for counselling services to be available to my participants if necessary (see Appendix E). Through the interviewing process, I spent between three and eight hours with each participant depending on how the participant was feeling. I did not want to push the participants, and ensured they were comfortable throughout this process, observing their body language and the tone of their voice while they were telling their stories of experience. I took on the role of active listener (Seidman, 1998), and as an "emic" [insider of the culture] (Patton, 2002, p. 267), injected insight 
into the culture, the setting and people who were included in the stories, with the intention of enhancing the accounts provided to be inclusive of emotions and tensions and to achieve richness in each of the swimmers' recollections. All interviews were audio-taped with prior consent obtained from the participants.

I then constructed the taped recollections into narrative non-fiction accounts by means of "an elaborate text building strategy which rested most fundamentally on the existence of a very strictly drawn and very carefully observed narrative contract between writer and reader" (Geertz, 1991, p. 58). When [re]constructing the stories as they were actually told, I construed the Australian swimming culture as a domain where different social activities took place whether it occurred between athlete and coach, athlete and athlete, coach and parent, or athlete and parent. I utilized devices found in ethnographic genre such as performance text (Denzin, 1997, p. 90) which can be referred as a way "of shaping an experience without losing the experience; it can blend realist, fictional, and poetic techniques; it can reconstruct the sense of an event from multiple 'as lived' perspectives; and it can give voice to what is unspoken but present" (Richardson, 1994, p. 522).

Once the [re]constructed stories were completed, the participants were asked to read these versions [interim storied accounts] to ensure that their accounts had been re-constructed in a manner that accurately and fully represented the events, experiences and emotions that they remembered. This is also referred to as member-checking (Seidman, 1998) and involved a series of communications, achieved predominantly through telephone conversation and email until each participant was happy with the intricate details that had been included. In this process, one of the participants found the storied [re]constructions prompted her memory and consequently after reading each of her narrative accounts, she then provided me with further detail than the initial taped conversations. This process with her was lengthy and it took several attempts until she was completely happy with the intricate details that had been included. The ongoing "narrative repair" (Robinson \& Hawpe, 1986, p. 123) that took place, thus provided a richness and multi-layered texture to the stories of experience that would not have been achieved had the "member checking" not been so thorough. 
As a participant-researcher, I also acknowledged my ethical responsibilities in regard to what I chose to disclose in some of my personal stories. Several of my own initial autoethnographic accounts had distinctive features and private conversations relating to my family. However, I acknowledged that while the stories were 'my data,' they implicated others, whose consent I had not sought to be involved in the research and who I needed to therefore show personal and professional concern for. I therefore chose to remove certain features and details of conversations due to the private and intimate nature of the storied accounts and the ethical issues that can be associated with the revealing of such information.

As I discuss further below, the act of participation in the interviews and the dialogue that featured throughout the research process did have an emancipatory dimension, specifically enabling myself and the other two participants to gain a degree of clarity in relation to past and current events, experiences and emotions that they had not previously achieved. The entire process from commencement of interviews until the participants were happy with the re-constructed stories took approximately 18 months. During this time, my social and emotional relationship with the participants changed, evolved and grew. At the beginning of the data collection process, the three of us had shared the common bond of swimming and competing. However, over the course of the data collection process a trust in each other - a bond of sympathy, camaraderie developed, so much so, that now - a strong connection, almost spiritual in nature has occurred. Below, a voice from one of the participants at the conclusion of data collection:

"I'm going to be a bit soppy here. I just wanted to say I'm so happy we are in touch again. I realize that after all of our discussions that we have had a similar childhood. Even though we have known each other forever, I never knew and I guess I feel a great connection. You are truly a great friend."

In employing autoethnography, I relied upon depth in self-reflection to generate my personal stories. Autoethnography is thus a qualitative research method, which allows me to write in the first person, connecting my personal experiences to the cultural (Ellis \& Bochner, 2000, p. 739). Autoethnography is the act of writing about (my)self refracting elite sporting culture. Ellis and Bochner (2000) define autoethnography as: 
an autobiographical genre of writing and research that displays multiple layers of consciousness, connecting the personal to the cultural. Back and forth autoethnographers gaze, first through an ethnographic wide-angle lens, focusing outward on social and cultural aspects of their personal experience; then, they look inward, exposing a vulnerable self that is moved by and may move through, refract, and resist cultural interpretations. (p. 739)

Ellingson (2006, p. 304) noted that autoethnographic narratives "offer embodied details and problematize the production of knowledge, and reveal the profane in the sacred process of research." Yet, it seems important to acknowledge this as an intention rather than assured feature of autoethographic narratives. In so doing, autoethnography allows me to excavate my own experiences as an Australian representative within the elite swimming culture, aligning with Richardson's (1994) assertion that "autoethnographies are stories about events that really happened to the writer"(p. 931). A way to position the body as a producer of knowledge is to write autoethographically (Ellis, 1995), therefore, autoethnography allows me to write from multiple positions within the lived experience, injecting my personal experience into my stories and then reflecting on that experience. Thus, autoethnography allows me to "reflect on and critically engage with my own participation within an ethnographic framework" (Tedlock, 2005, p. 467).

My stories, as those of the other participants, would be shaped and inherently limited by my willingness and ability, as a research participant, to provide detail and depth of insight into highly personal emotions and experiences. In this sense, autoethnography is acknowledged as according the researcher-asparticipant an important element of authority in and control over the research process. Arguably, this is to a degree not shared by the other participants in this study, as I did not experience prompting and probing in the way that the other participants did in the course of the collaborative generation of their stories. Yet, self-dialogue was a strategy and feature of the writing process as I generated stories which captured emotion and documented sub-conscious thought and 'talk.' While for two of the participants, the process of producing the stories was a negotiated process involving dialogue between the researcher and participants, for 
my story, the negotiation was 'with self,' and was a process that needs to be recognised as similarly, giving rise to tensions from a participant perspective in relation to what one will choose to, and take the opportunity to, reveal 'as data.'

\section{Presentational form: Voices and vignettes}

Connelly and Clandinin (1990) have stated that, "humans are storytelling organisms who, individually and socially, lead storied lives" (p. 2). Yet, storytelling in the context of this research and specifically with agendas associated with avoiding inequitable power-relations in research while maximising prospective audience engagement, is far from a simple act. As a researcher and participant, I found it difficult for lived experience to always be organised into long storied accounts. Like Austin (2005) when discussing autoethnography, to fully capture "the multiplicities and tensions of identity," she found that she needed to consider the use of "more innovative and creative forms of presentation" (p. 25). Two methodological agendas were closely tied at this point: the intention that readers would be able to vicariously share participants' experiences and emotions, and for participants to not be restricted in their telling and re-telling of their lived experiences. In addressing these issues I drew from Richardson (1997), who discusses not limiting a story to one narrative. She says:

everyday life experiences are not organised around the long biographical account, the epic poem or the life history. Rather, people tell stories about events in their lives; the meaning of the event changes through the invocation of different implied narratives. Not all events, further, are stuffed into the same narrative. A life may have a 'plot line,' but not everything lived - nor everything of import to the person - fits neatly into a 'plot.' We are not characters. Our lives are not morals. They are not even ethnographic narratives. (p. 181)

My approach, therefore, was to generate and support the other participants in generating a series of vignettes, each centring on what from a participant and a research perspective, could be deemed key events or experiences. As described above, the process of achieving depth and detail relating to each vignette was 
progressive, collaborative and negotiated. In developing and organising the vignettes for publication, I returned to the issues of voice (seeking representation to 'the swimmer voice' and 'the body voice' in and through the vignettes) and the matter of audience engagement. I also grappled with the representation of my own voice as researcher, not only participant. A theatrical mode of presentation was identified as offering a means of addressing these multiple agendas in writing and representation of data as it conveys a sense of realism that prose ultimately could not achieve. My desire to represent the stories of the participants in this form was also fuelled by the distinct lack of representation of swimmers' lived experiences in literature. Specifically, this format would give the stories the 'centre stage' that it was felt they deserved, enabling me to be represented in writing as both a participant in and narrator of 'the production' and hopefully, present the data in a manner which would achieve a high degree of audience engagement.

In this thesis, the vignettes have thus been organised to collectively form three Acts of a play that reflect the central research theme of body pedagogies. The first and second Act in the play doubles as a prequel ${ }^{13}$, revealing the three main characters at the time of their participation in the Australian swimming culture as adolescents. For all of the actors, this part of the production involves retrospective recall of and reflection upon events that respectively occurred for the participants in the 1970s, 80s and 90s. The third Act in the play represents a sequel $^{14}$, a continuing narrative of Act one and Act two, composed by the participants as adults and focusing upon their present day (2009/2010) lives and feelings relating to the theme of body pedagogies, which the play is based on. At the beginning of each Act, a prologue features, providing the audience with a preface, a foreshadowing, some background information relating to the three actors and their lives around which the Act is based. The Acts are consciously staged as the actors' own production, seeking to maximise their authoritative voices. The storylines told by each of the actors are their recollections of Australian swimming culture, as lived and experienced by them, as adolescents and in present day life. As the narrator, I am able to provide further insights by

\footnotetext{
${ }^{13}$ Prequel: "a work (as a novel or a play) whose story precedes that of an earlier work" (Merriam-Webster Online Dictionary, 2009).

${ }^{14}$ Sequel: "subsequent development, the next instalment (as of a speech or story); especially: a literary, cinematic, or televised work continuing the course of a story begun in a preceding one" (Merriam-Webster Online Dictionary, 2009).
} 
utilizing my voice/s - reflective and analytical voice, broadening the re-telling of the stories by at times revealing my own voice and feelings within (and in response to) the other swimmers' [actors] stories.

The vignettes that shape each Act have a number of distinctive features. They capture language occurring from multiple dimensions along with the feelings, thoughts, tensions, and inner monologue/s of the participants, with the intention of provoking reader emotion [evocation]. Each vignette seeks to provide new insights into the life of a swimmer and also as an adult woman [postswimming] with the use of vernacular language with the intention of provoking dramatic potential. The main force, focus and tension of each vignette is created through the actors' dialogue, and there is far more to this than merely the expression of achievement or disappointment as a swimmer. Relationships, inner thoughts and feelings are expressed through the language of hope, fear, friendship, fantasy and realism that sees the swimmers' resourcefulness in overcoming frustration, disappointment and failure to meet expectations of themselves and those closest to them in the Australian swimming culture (coach/parents/other swimmers). The intention is that the reader will be able to feel empathy with the swimmers, relating to and in a sense '(re)living,' while not enduring, their experience. The stories were intended to allow readers to momentarily 'become the body,' 'be the body' of the participants, whether it is the adolescent elite swimmer body, the comeback body, the 30-40 year old body or the performing body. Thus, resonance is immediate and direct "providing [sic] powerful access to the uniquely human experience of time in five sociologically significant ways: the everyday; the autobiographical; the biographical; the cultural; and what I term the collective story" (Richardson, 1990, p. 22).

\section{Fore grounding the swimmers and their body's voice}

Trinh (1999) states: "we write - think and feel - with our bodies rather than only with our minds or hearts. The body and the person-self are one" (p. 258). Thus, in this research, the body is a site of writing and the stories presented are deeply embodied, reflecting as Jackson (1990) has highlighted, that personal histories of 
social practices and relationships are inevitably (and we argue, very powerfully) embodied in our everyday embodied form, movements and interactions.

Representations presented in the chapters that follow are designed to powerfully reveal the ways in which my body and the participants' bodies became disconnected from ourselves in what appears to be the surrendering of her body's voice and power to our coaches, our parents, and Australian swimming. The style of representation adopted provided a means via which I could address the concern that the swimmers' body's voice would be employed as a voice of authority in the research and thus presented as the primary data.

\section{The individual and collective voice}

Sparkes (1997) revealed that he was not willing to shelve his knowing through his research. I take up Sparkes's (1997) method and apply it to my role as researcher and participant. In doing so, the three Acts excavate our stories of experience, revealing my own knowing/knowledge as researcher/participant and that of the two other participants. Accounts are on the one hand, highly personal, clearly articulating the embodied experiences and lived emotions of the participants as individuals. At another level, however, and a crucial characteristic in considering the notion of empowerment in this research, the stories serve to present and represent "a collective voice" (Richardson, 2000) in relation to the cultural practices or "memes" (Dawkins, 1989) that the three participants experienced, were subjected to, and participated in. Dawkins (1989) uses the term "meme" to refer to the spread of cultural ideas and practices, which can be transmitted from one mind to another through speech, gestures and rituals. Many of the stories capture more than the participant's individual experience of specific cultural ideas and practices. Collectively, they also convey the transmission of ideas and practices within the culture of Australian swimming. Through 'relational analysis' (Kirk, 1999) by 'connecting the dots' (Klein, 2000), the long term affects of the memes have also been pursued in this research. The theatrical presentational form, featuring vignettes, was chosen in part to vividly and emotively convey the memes and their affects, with the aim of extending sociological understandings of cultures (the elite and sub-elite culture of Australian swimming) that have 
remained somewhat opaque and largely closed off from critical academic inquiry. In taking this approach I drew from Harvey (1990) who says:

Knowledge does not reside in a cupboard or on a bookshelf to be taken out, dusted down and looked at. Knowledge exists in our everyday lives. We live our knowledge and constantly transform it through what we do, as much as it informs what we do. For critical social research, this means that an analysis of oppressive structures is itself a political act. Knowing cannot be shelved; it becomes part of our lives, and informs our action which engages structures (Harvey, 1990, pp. 22-23).

The following extract provides an example of how my combined swimmer and academic knowledge came to the fore in my dual role as researcher and participant.

"As I get to the doorway, I peer inside. I notice that the managers have an efficient system in place. The physiologist is taking the skin fold measurements and one of the team managers is recording the information onto a clipboard. I see the swimmers in front of me who have been measured move to the other side of the room where they then ready themselves to get their weight recorded. A coach is taking the weight readings and the other manager is recording the information. I cannot believe how quickly they are getting through the line, the swimmers move so efficiently around the room - robotic like from one measurer to the next. It looks like a factory production line” (A story from McMahon, 1991 - 16 years of age).

An inherent intention here is 'reader impact,' acknowledging that the very act of reading, feeling and experiencing the body of the participants as articulated in stories can be likened to what Ropers-Huilman (1999) refers to as 'witnessing.' For Ropers-Huilman (1999, p. 23) acts of witnessing occur "when we participate in knowing and learning about others, engage with constructions of truth, and communicate what we have experienced to others" (p. 23). As a consequence, the stories of others can affect the reader greatly. Ropers-Huilman (1999, p. 24) discusses this saying, "our bodies, hearts and souls are changed and renewed by 
what we witness in our lives. Witnessing is powerful." Notably, Ropers-Huliman (1999) acknowledges that there are both "great opportunities and dangers inherent in the process of witnessing others' lives and constructing meanings about those experiences" (p. 24). As a researcher, I recognise the need to therefore be cognizant of the prospective dangers as well as the opportunities inherent in my endeavour to pursue 'witnessing' in and through my methodological approach and presentational strategies. Notably, Sparkes (1999) also comments on the potentiality of reader impact through the use of narratives saying, "well crafted narratives of self are able to evoke an emotional response in the reader, enhance their empathetic understanding of selected issues, and enable them to reflect upon their 'actual worlds' from different vantage points" (p. 24).

\section{Power-relations, empowerment and emancipation}

In this final section of discussion, I focus on issues that were embedded and explicit in my methodological concerns, relating to power-relations in research, researcher-participant relations and the matters of the purpose(s) and perceived value of research. For Boog, "the concepts of emancipation and empowerment are closely connected with the concept of participatory democracy" which can be described as "communication and participation in decision-making" (Boog, 2003, p. 428). In this instance, the focus is upon the extent to which and manner in which communication between the researcher and participants was maintained throughout the research process, and the opportunities that were provided for them to be involved in decision-making during the research process.

As indicated above, my position as researcher-participant inevitably set me apart from the other participants. While I constantly endeavoured to emphasise collaboration and consultation, this was always pursued from a position of relative authority as researcher, writer and ultimately, narrator of others' stories. Yet, from that position, my goal was precisely to ensure that the stories remained 'others' stories,' representative of their very personal experiences and emotions. It is inappropriate to pretend that empowering participants in the production of narrative accounts is either easy, or necessarily the path that participants will wish to pursue. In the early stages of the research, I struggled with the participants' 
difficulty in grasping the potential and processes of ethnographic narrative.

Sharing some of my own stories was one way in which I could both demonstrate trust as a co-participant and show the possibilities in this form of representation. As described above, throughout my communication with participants (pre-, during and post- interviews) I engaged in an ongoing process of consultation and negotiation in relation to both what the focus of each participant's stories would be, and how they would be presented.

Two strategies were employed in developing and presenting the stories, specifically to ensure that while 're-created,' as far as possible the stories represented and conveyed the events, experiences and emotions as they had actually occurred. The first strategy was an "emotional recall" strategy proposed by Ellis and Bochner (2000, p. 752). Using visual prompts such as pictures and video footage from the time the swimmers were training and competing, the participants visualised their presence (physically and emotionally) back in the respective scenes. The intention was that they each 'became a competitive swimmer again,' revisiting and reliving experiences as adolescents participating in their training environment, training camps and Australian teams. Ellis and Bochner (2000) state, "if you can revisit the scene emotionally, then you remember other details" (p. 752), a point that is born in the stories generated.

In the recalling of the adolescent experiences, there was a significant period between the events and the re-telling of them. Participants were therefore able to reproduce stories with the benefit of distance and greater experience of life, but also with the acknowledged potential for memories of events and experiences to be such that the experiences and events are now presented in a particular and partial way.

The second strategy related to the latter concern. Ellis and Bochner's (2000, p. 751) "reliability check" was used to authenticate and potentially add depth and scope to the participants' stories. This involved the sharing of 'partial writes' of experiences, where as researcher/participant, I conferred with other swimmers, coaches and physiologists of the same era, to seek their own accounts of the experiences reported by the participants and to thereby ascertain resonance with those stories or equally, identify its absence. Potentially, this process is disempowering from a participant perspective, inherently shedding doubt on the 
authenticity of their stories. The participants were hesitant but ultimately accepting of the rationale for seeking external validation of their stories. The prospect of stories being shared with another swimmer from the same era caused one of the participants to react very openly, with worry and fear. She was particularly concerned about her identity being exposed. For me, this situation gave rise to clear tensions, as she was committed to respecting participants' views and protecting them from risk or harm as participants in the research, while also remaining acutely aware of the pressures that she felt to seek further validation of her data. I therefore adjusted my approach and in the case of this participant, sought agreement for the participant's husband to be involved in the 'reliability checking' of the data. The second participant was more forthcoming in wanting and being willing to take ownership of the validation process. She shared her stories of experience with another swimmer of her choice from the same era, and then shared the points arising with me as the researcher. In relation to my own stories, I sought out a physiologist, two swimmers and a coach from the same era as a method of testing the validity of her data. This was done through partial writes of experience, conferring with those identified as being from the same era of Australian elite swimming. They in turn confirmed a verisimilitude and resonance with her stories with their own reports of their experiences. I saw this process as a necessity within the discourse of academia; however I felt some unexplained hesitation throughout it. Even though, an 'other' was utilised in checking the re-constructed stories, it is important to recognise that as the researcher/participant in this production, I do not imply or make claims about the generalisability of the data generated to all individuals involved as participants in Australian elite and sub-elite swimming. Rather, I highlight that in my experience none of the participants are lone voices and as a consequence, the stories do, arguably have an impact that exceeds that achieved through any of them considered in isolation.

\section{Clarity and meaning}

For Watson (2009): 
narratives are important to us not only or even primarily because they tell about our past lives, but because they enable us to make sense of the present. We attempt to create coherence and give meaning to our lives by learning to read time backwards. (p. 469)

These points are particularly pertinent to this study because of it longitudinal dimension and the underlying aim to provide the opportunity for participants to gain clarity and meaning in relation to both their adolescent and present day experiences and emotions. Stanley (1993) contends that through the employment of narrative "post hoc understandings" (p. 43) can be achieved as they "take snippets of their life, sort and ponder [sic] them as a way of coming to draw conclusions or understandings about the self." Through the actual process of telling, participants have the opportunity to sort and ponder their lived experiences, "retrospectively revising [sic], selecting and ordering past details in such a way to create a narrative that is coherent" (Polkinghorne, 1988, p. 106). Welty (1984) illuminates Stanley's thesis of "post hoc understandings" saying that:

Writing a story or a novel is one way of discovering sequence in experience, of stumbling upon cause and effect in the happenings of a writer's own life. Connections slowly emerge. Like distant landmarks you are approaching, cause and effect begin to align themselves, drawing closer together. Experiences too indefinite of outline in themselves to be recognised, for themselves connect and are identified as a larger shape. And suddenly a light is thrown back, as when your train makes a curve, showing that there has been a mountain of meaning rising behind you on the way you've come, is rising there still, proven now through retrospect. (p. 90)

I did not presume that emancipation would automatically be achieved through participation. However, I hoped that through employing narrative ethnography and autoethnography, I would achieve this. For Polkinghorne (1988, p. 11) narrative [story telling] is "the primary scheme by means of which human existence is rendered meaningful." It also has the potential to challenge disembodied ways of knowing by enhancing empathetic forms of understanding by seeing our 'actual worlds' more clearly (Sparkes, 1999). Through the process 
of storytelling, I hoped that the participants would be able to "organize" their lived experiences "into temporally meaningful episodes" (Polkinghorne, 1988, p. 1) thus potentially crystallising their thoughts. As Sparkes (1999) explains:

As individuals construct past events and actions in personal narratives, they engage in a dynamic process of claiming identities, selves, and constructing lives. Furthermore, how individuals recount their history (what they emphasize or omit) has a direct bearing on what they can claim of their lives (p. 19).

The participants as individuals potentially have the ability to draw some conclusion, some understanding to what they have encountered in their swimmer lives and in their lives post-career. Fetters (1978, p. 44) further resonates:

If man is to live as a creative enterprise, he must begin to communicate with himself, to enter into open dialogue with himself. He must trust his own experience and what his experience is revealing to him. He must face the realization that social reality is a human construction and that the myths, the pervasive stories, images and symbols of his culture have structured much of his experience. He must have the courage to confront the deep sense of abandonment that comes with momentarily suspending and questioning those cultural myths, so that he might be responsively open to alternate ways of being in the world.

If I translate this gendered language, then Fetters' thesis is salient to this thesis. Appropriately, in considering the extent to which the methodology and research process succeeded in generating emancipatory potential, I rely upon the participants' voice.

\section{Carly (February, 2010)}

Participating in the research and re-living some of my adolescent experiences as a swimmer was painful. It brought back memories that I had buried deep down inside for a long time. I cried. I cried a lot. Being able to see my swimmer experiences in writing, even though it was painful, I came to learn to understand. Things became clearer. In a way, I was able to see 
my life from the perspective of an outsider. I was able to see it through adult eyes and this helped me. Most of all, it helped me to recognize why I am the person I am [as an adult woman], why I react the way I do and why I battle with those negative thoughts on a daily basis. I no longer have to think, "why me" as I did when I was a teenager, but am able to have insight into who I am.

\section{Jenny (February, 2010)}

My research journey began in 2007. This was the time when my cathartic journey also began. If I look back to that time three years ago, I reflect on a woman who could not recollect a story from her swimming career without crying, without feeling pain in her body. I reflect on a woman who could not look in the mirror without cringing, whose eating was out of control in what she thought was an act of rebellion. If I look to the now - in 2010, I see a woman who understands, a woman that realizes the damaging potential of a culture, a woman who is finally at peace with her body and can write and read about her life and have 'light bulb moments'-moments of clarity rather than falling into a cycle of depression. I have finally found peace and that I attribute to my cathartic journey - my research journey.

\section{Mandy (March, 2010)}

When I re-read my stories from when I was swimming, two emotions overwhelmed me - sadness and anger. Even reading the stories as an adult woman, I find it hard to believe that those things happened to me. I was only 16 in most of the stories and did not have the power to do anything about it. What I learnt most from this experience is the power that I have in my current position as a swimming coach. My words, even the simplest of ones have the potential to cause damage to my athletes in the long term; my actions have the potential to do the same. From this experience, I now look at how I interact, the relationships that I have with my athletes because there is no way that I want them to go through the hurt and pain that I have lived with since my adolescence. So, this experience has led me to understand - an understanding that I am in a position of power and have 
the potential to affect my athletes. This is something I will do everything to avoid and give them a positive experience - something that I never had.

The voices of myself, Mandy and Carly, in the present day reflect catharsis and in so doing resonate with Foucault's thesis (1997, p. 232) where the act of telling is a practice that "permits [sic] individuals to effect...operation on their bodies and souls, thoughts, conduct and way of being so as to transform themselves in order to attain a certain state of happiness, purity, wisdom, perfection or immortality." Their voices in the present day, in post research exemplify emancipation as they have all gained some new meaning and understanding of their experiences. Thus, the participatory experience of myself and the two other swimmers can be summarised by Ricoeur (1981, p. 170) who says, "looking back from the conclusion to the episodes leading up to it, I have to be able to say that this ending required these sorts of events and this chain of actions." Ricoeur's (1981) perspective is thus reflected in the voices of myself, Mandy and Carly in the present day - in post-research. For Pynchon (1975, p. 306), "the fiction of continuity, the fiction of cause and effect, the fiction of humanized history [becomes] endowed with reason" thus exemplified in our telling in the present day.

\section{Conclusion}

This chapter has described and critically analysed the ways in which narrative ethnography and autoethnography have been employed in a research project that is acknowledged as addressing sensitive and for some people, problematic aspects of sporting culture. It has sought to demonstrate the complexity, rich potential but also considerable challenges inherent in employing these methodologies 'with specific agendas,' and particularly, with the intention to empower participants in and through the research process while simultaneously, accepting (and seeking to pursue) parallel interests in relating to aspects of the culture that has been the focus of this research. Time will tell whether the publications arising from this research achieve the degree of audience engagement and 'impact' that as a researcher and academic I have sought in and through our participation in this research. Specifically, for the research to serve to raise new questions about and 
extend understandings of sporting cultures, the ways in which they are experienced, 'lived' and 'lived out' by participants in those cultures. Ellis's (1995) notion of 'evocation' has been used in this research for validity, authenticity and for impact in relation to these specific research agendas. Those agendas are openly acknowledged as mine, but are also stressed as ones which from the outset, the other participants in the research were not merely aware of, but also accepting of in agreeing to participate. 


\section{CHAPTER FOUR ${ }^{15}$}

\footnotetext{
${ }^{15}$ Chapter four features the actors' stories of experiences. The chapter comprises of three Acts. The first Act [prequel] features body pedagogies occurring within the discourse of perfection as adolescents. The second Act [prequel] features body pedagogies occurring within the discourse of performance as adolescents. While the third Act [sequel] features body pedagogies as adult women.
} 
ACT 1

TALKING SWIMMER BODIES: EXPOSURE TO A BODY FOCUSED CULTURE? 


\section{ACT ONE - Prequel}

Prologue: The stories told by Carly occurred when she was between ten and fourteen years of age. She is a club swimmer who has had success at a local and state level. My [McMahon] stories of experience occur over a ten-year time frame within the elite discourse of Australian swimming. Mandy's stories are experiences that occurred on national representative teams, also within the elite discourse.

\section{Carly}

"10 years old"

State titles are only four months away. Most of the senior swimmers in my training squad are heading off to the Olympics in just four weeks so we are all in heavy training. Even though I am 10, my coach includes me in all of the sessions and I make sure that I never let him down, training as hard as I can in all that he gives me. I like that he gives me the same program as what the older swimmers do - his champions. That way I know I am doing what it takes to win an Olympic gold medal.

It is Saturday morning. In the middle of our main set of 10x200's butterfly on three minutes, I hear some of the other girls talking about having to weigh-in and getting a skin fold test but I am not quite sure what it is that they are talking about? They seem worried as they discuss it so I immediately think that it must be something terrible that Tom makes them do. I strain to hear two of the older girls talking.

Female team mate: "I hope I have not put on weight again. Am sick of doing so much running."

As I climb out of the pool at the end of the session, a lady that I have never noticed before enters the pool complex. She is carrying bathroom scales, these strange metal things and a clipboard. She heads straight over to where Tom is standing, in a purposive manner - as if she has an important job at hand. I see 
her talking to him but I cannot hear what they were saying. As I put my towel around me, I can see her writing down the names of swimmers who Tom is pointing to. I assume that they must be the ones that are getting measured.

I see Tom point to me. This surprises me. Instead of being worried like the older girls, the whole process intrigues me. I am glad that Tom has chosen me.

As I finish drying myself off, Tom comes over to where I am standing and tells me that I am getting measured today. He points to where I need to go so I head straight over to where the bathroom scales are. The measuring lady is standing behind them. I climb on.

Measuring lady: “38 kilograms.”

She writes my weight down onto her clipboard. She then grabs the strange metal things and a measuring tape. She measures some different points on my body and uses a black pen to draw a mark. She does this in seven different places. She then pinches the metal things onto my skin. It hurts but I don't let anyone know my discomfort.

Carly: "What does this do?"

Measuring lady: "It measures how much body fat you have. The less fat that you have the faster that you will swim."

When she finishes pinching my skin, she tells me that my percentage body fat is seven percent.

Measuring lady: "You're as low as Katie."

Even though it is the first time that I have been tested, my competitive nature comes out.

I want to be the lowest.

I am going to beat Katie next week.

I hear some of the other girls' results. They are not as low as me. I hear the lady say: 
Measuring lady: "You have increased since last week!"

I then see Tom who is within hearing distance walk over to those girls.

Coach: "You have put on weight! Champions don't put on weight. Better run it off."

They are not allowed to go home.

Tom: "Off you go. You have got 16 kilometres. Need to burn off that fat girls!"

They have to run 16 kilometres around the aquatic facility. I realise that the 16 kilometre run is a punishment that everyone has to do if they put on weight. It is a punishment that I will have if I put on weight.

I better not put on weight.

As I grab my bag ready to walk out the door, my coach yells at me,

Coach: "Smith, you are not finished! You've got four kilometres."

I realise at that moment that I have not escaped a run entirely. The people who do not put on weight still have to do four kilometres.

The measuring process that intrigued me an hour ago no longer seems exciting.

\section{Carly}

"11 years old"

It is week two of hell. Even though this is only my second time on hell week camp, I now feel so experienced. I finally have had the opportunity to experience what many of my teammates have talked about for so long. Last week was hard. It was hell. The camp lived up to its name. I am so exhausted.

It is 2.30 in the afternoon. I meet some of my teammates who are running to the pool. It is six kilometres away. My coach's words play over and over in my head as I prepare for the run. 
"If you want to be a champion, you need to do extra and that includes running to the pool."

As my weary body begins to pound the pavement with some of my fellow teammates, I feel a sense of achievement, as I know that this training is like depositing extra money in the bank, something my coach talks about all the time. Last week, I would have deposited more money than any of the other 11 year olds that I race. As I get into a rhythm, one of the other swimmers suggests that we stop for an ice cream at the corner store. We all agree that it would be a great idea so we all stop. After standing at the freezer for several minutes, I finally make my choice - a Golden Gaytime.

Outside the shop we all enjoy the ice-cream. As we are eating it, the white team bus with the swimmers who didn't run passes us. My coach is driving. As it starts to disappear down the street, some of the boys hang out the bus window and yell.

“Busted!"

I become panicked.

Hope my coach didn't see me otherwise I will cop it.

It is only a couple of minutes before we are all finished eating our ice-creams and we finish running the remaining four kilometres. As I enter the pool gates, I automatically begin my stretching routine. The rest of the swimmers do the same. The boys who were on the bus start to tease me for eating the ice cream. Their taunts panic me.

I hope my coach did not see me.

I hope the boys don't tell him!

After 30 minutes of stretching, I ready myself to get into the pool. As I approach the end of the pool, my coach's eyes connect with mine and he says,

Coach: "Did you enjoy your ice cream? I hope it was worth it"

Oh no, he did see me!

I get angry because I notice that I am the only person he says anything to. 
Why am I the only one who always gets into trouble?

As I dive into the pool, I wonder how I will be punished.

Later that night, as I am climbing into bed with a full belly of mince from dinner, I recall the events of the day. I am surprised that I escaped punishment for eating the ice cream. It feels like my eyes are only closed momentarily when I hear banging on the window and my name being called. I look at the clock beside my bed and it is $11 \mathrm{pm}$. It is pitch black. The banging on the window continues. I realise that it is my coach.

Coach: "Get up Carly, you need to run off that ice-cream you ate today! Get your running shoes on; you haven't burnt it off yet!'

Carly: "What do you mean? It is 11pm?"

Coach: "Get your running shoes on and get out here! You haven't burnt off that ice-cream yet!"

My eyes fill with tears. As I pull my running gear, fumbling in the dark, my teammates sleep soundly. I find everything but my socks so I go without them. I suddenly start to feel singled out. I become angry. When I appear from my dormitory, I notice the assistant coach is also outside. I look directly at both of them and say,

Carly: "I hate you!"

They ignore me and don't seem to care what I just said.

Coach: "You've got 11 kilometres kiddo! Need to burn off that ice cream you ate."

Clay is running with me. As we start running, I am cautious even though I am not alone. The darkness makes the ground seem unsteady. After about 20 minutes of running, my feet become so sore from not wearing socks and from all of the other running that I have been doing. Feels like I have got blisters.

Carly: "Clay, can we stop? I have got blisters!"

Clay: "Well take your shoes off then. We are not finished!" 
I take my shoes off and Clay carries them. I am so angry.

Carly: "Why am I the only one being punished?"

Clay: "Keep up Smith or you will have to do this again when you get back."

I run about five metres behind Clay the rest of the way. My stomach feels heavy, still full of mince from dinner. My tummy gurgles. Feel like I am going to throw up.

Carly: "Why am I the only one who has to run? I am not the only one who ate an ice-cream?"

He does not answer. After an hour of running, I arrive back at the camp exhausted. It is midnight. I climb into bed for the second time that evening, joining my teammates who have been asleep for over three hours.

I shouldn't have eaten the ice cream. I am such a fat pig.

I cry myself to sleep.

(McMahon, Penney \& Dinan-Thompson, in press).

\section{Carly}

"Another story as an 11 year old"

\section{Part 1}

It is my first national age group championships. I think I like Canberra as I have had fun competing here. As I sit in the grand stand with my teammates around me on this final evening of competition, I laugh and enjoy their company. As the man on the public address system starts to speak, our focus turns to what he is saying rather than each other. We all wait in anticipation as he calls out the names of a development team that has been selected from performances at the titles. The team will travel to Sydney. I listen intently and hold my breath to see if my name gets called. One by one, a name is called, systematically, in fact alphabetically. As the letter ' $S$ ' approaches, my heart starts to beat quicker.

Announcer: "Carly Smith" 


\section{Yes, I made it!}

I am so happy. I am off to Sydney for a training camp in three weeks time.

The three weeks pass quickly and before I know it, I am farewelling my parents as I board the plane. Ifeel a sense of excitement because at 11, this is my first trip away from Mum, Dad and my coach. As I settle myself into my seat on the plane, I look around at the other swimmers who are also travelling with me and I realise that I am the youngest. I smile to myself as I cannot wait for the ten days ahead. A sense of freedom overcomes me as I sit there with just team mates. It feels like the control has been lifted from me.

On the first day of the training camp, I am surprised by how different the environment is to my home environment. It feels like I am on a holiday. I now truly understand that saying that I have heard many times before, "a change is as good as a holiday." After completing the first pool session, I realise just how different some other swimmers train. The coaches use such different training programs to the ones that Tom uses. It is interesting, even fun. I only had to swim 12 kilometres today compared to the 18 kilometres that I usually do at home. I find everything different, including the conversations I am having. It seems that they are not focussed around swimming and competition even though we are all here for that purpose; rather I find that we are tending to talk about boys and clothes. I find myself laughing, more than I usually do at home. This is so different to the total swimming focus at my house.

That evening after I finish training, I follow the rest of the swimmers into the food hall. As I walk in, my eyes nearly pop out of my head. I see food and lots of it. I cannot believe how many choices I have, so many delicious choices. It is nothing like what Mum cooks me at home and the best thing is that I get to choose what and how much! It gives me a real sense of freedom, even control. I eat a huge plate of pasta, followed by rice pudding for dessert. I have a mug of warm hot chocolate and a biscuit for supper.

$$
\text { Ifeel free-no control. }
$$

I like having choice. I like being able to eat some more if I am still hungry. My tummy feels full, a warm feeling of contentment. The full feeling is very satisfying 
and as I finish off my warm drink, I find it hard to recall the last time that I felt this 'full' feeling.

As I sip my last mouthful of hot chocolate, I notice how the rest of the squad are still lingering in the eating area. They are all joking around and laughing. It is so much fun to be around them. I listen to their every word, every joke. Some of the older swimmers decide that it would be hilarious if we started a dare competition - an evening ritual after everyone has finished their dinner.

Male teammates: "Smith, you are up first. We dare you to eat three sticks of butter!"

My competitive nature takes over. Saying 'no' is not an option.

Carly: "Alright, that is easy."

I eat them. The older swimmers giggle as I do. Then it is Sarah's turn.

Female teammates: "Sarah, I dare you to eat four bowls of custard!"

Sarah is as obliging as me and does it. At the end of dinner as we walk back to our dormitory, I feel much older. I don't feel like that I am 11 anymore. It feels like I am 16, like the other swimmers. I can swim as fast as them and now I showed them that I can complete any challenge. It is though I have been accepted - I am part of the team.

For the nine days that followed that first evening, I ate and ate and ate, choosing whatever I wanted from the vast selection. But as the end of the camp starts to near, I begin to dread going home.

\section{Carly}

"Another story as an 11 year old"

\section{Part 2}

The last day of camp has arrived. To make it even worse, we have to get our weight and skin fold measurements done before we can go home. Even though I 
am used to having this done at home, Ifeel nervous because the entire mood of the team has blackened which I think has affected me.

We all line up in the gymnasium in readiness to get measured. The older swimmers move to the back of the line that has started to form. As they do, I am left standing at the beginning. I step onto the scales and look for 38 kilograms. It is my number, the number that appears on the scales every Saturday morning. I watch the pin swing around and to my shock; the pin of the scales doesn't stop at 38 kilograms but settles on 46 kilograms.

Carly: "These scales are wrong! I am 38 kilograms."

Male coach: "Well, these scales say that you are 46 kilograms. They are not wrong."

I notice that some of the other swimmers in the line are listening intently to what my weight is, my response and also the coach's response.

Carly: "They have to be wrong! I was 38 kilograms before I left."

I cannot believe that in ten days I have put on eight kilograms. I am going to be in so much trouble. I am fat. I cannot believe I have a weight problem. I am terrified about what my punishment will be. My coach does not like any of the girls to be over 45 kilograms.

What will my parents do?

What will my coach do?

I am going to have so much running to get back to a weight less than 45 kilograms.

Once the measuring is finished, the camp is over. We all say good-bye to each other. As we are hugging each other, reality sets in and a sense of fear overcomes me because of my weight. My farewells are subdued as my thoughts are focused on what will happen when my coach sees me in my togs.

Our flight arrives at home on time. I don't feel excited about being home. I don't want to see my parents or my coach. I wonder what they will say and what they 
will do. I take my time collecting my bag from the carousel. As I walk towards Mum and Dad, my eyes fix on them. I can see them looking my body up and down. Panic stiffens my body as I approach them.

Mum: "What the hell did you eat down there?"

I nearly cry. I do not get a hello or a hug. Instead, Mum asks me what I have been eating. It is at that moment that I realise that my parent's reaction would pale in comparison to what my coach would say. I guess I would be finding out in the morning at training.

The next morning, I pull my togs on for the first time since finding out that I am 46 kilograms. I feel embarrassed, ashamed of what my body has become, of what I have allowed it to become. Ifeel uncomfortable. My togs feel tighter than usual.

As I walk into the pool, it feels like everyone is looking at me. Everyone's eyes are fixed on me, most of all my coach. I am so embarrassed of what I have done to my body. As I walk towards where my coach and teammates are standing I catch some of what my coach is saying to some of the other parents that are standing there.

Coach: "How could she put on so much weight in ten days? What did they do to her down there? Look at how unfit she is. I should never have let her go. It was a mistake. This will be the end of her if I don't watch her weight closely."

My body stiffens. I try to ignore the gazing and the comments but I find it impossible. After finishing my stretching routine, I get ready to get into the pool. I don't want anyone them to see my bum. How am I going to hide it when it is hanging out of my togs? My togs are now too tight. I pull the seam across to cover it but they do not stretch that far.

I hope everyone does not notice me trying to hide it.

I am going to have to do so much running to get rid of this disgusting fat off my body. I hate running.

I am so fat and disgusting. 
I can't believe this is happening to me.

I hope I don't get my periods now that I have put on weight.

If I get my periods my body will change. My coach and my Mum have warned me. My coach's words come to mind;

Coach: "If you put on weight you will get your periods, then your body will change and you will swim slowly." Now it might happen.

Can't get my periods, I will get boobs and a big bum.

Please God, don't let me get my periods! I won't swim fast anymore if I get my periods.

\section{Carly}

"Another story as an 11 year old"

\section{Part 3}

At 46 kilograms, I do not feel like a champion. I feel like a failure. I dread going to training. This session is no different. As I strip off my baggy clothes that I hide my body with, I notice that my coach is watching me and then he begins to yell.

Coach: "You've got a big bum! Better go and buy some new togs that are bigger!”

I cannot understand why everyone is always looking at me.

Why are they looking at me?

I hurry myself so I can begin the session. I feel safer when I am in the water as my body is not on show. I am doing everything that I can to lose the fat. I am hardly eating and running everyday but still my coach is not satisfied. I just cannot reach the weight that I was before the swim camp. At the end of the session, I wait in the water momentarily wondering what the best way is to exit the pool so everyone does not look at me. I wonder how I can get to my towel without everyone looking at my bum. I find an opportunity when most of the squad are distracted, grabbing my towel and covering my body. I head to the change rooms and inconspicuously 
take my togs off, careful not to drop my towel and expose my body. I have carefully placed it around my body so my stomach, arms and bum cannot be seen. My fat is so disgusting. The showers are open and there is no privacy so I decide to skip it-haven't showered after training since returning from the swim camp.

I don't want them to see my fat.

I change into my baggy clothes, covering my arms, stomach and legs. I am huge. As I exit the change rooms, I hear my coach yell at me.

Coach: "Smith, you are not finished. You have stairs". Have to burn that weight off that you have put on!"

I wonder when this nightmare will end. Will I ever be skinny again?

\section{Carly}

"14 years old"

\section{Part 1}

I wake from my compulsory afternoon nap with a grumbling stomach. I look at my clock radio beside my bed. It is still an hour before we have to leave for training. I am so hungry - really, really hungry. I cannot hear any noise outside.

I wonder if Mum has gone somewhere.

I quietly walk outside and I see Mum on the couch. She has fallen asleep.

I wonder if she will hear me if I go to the fridge?

Have to get something to eat.

I tiptoe to the fridge, quietly opening the door. I reach inside, carefully opening the bag the bread is in. I grab a piece. I then reverse the process by quietly shutting the fridge door. I shove the whole piece of bread into my mouth in the

\footnotetext{
${ }^{16}$ The aquatic facility where Carly trained had spectator seating that was situated over two levels. Her coach, as a method of controlling the swimmers weight would get them to run up and also down the stairs. There were 13 rows of stairs over the two levels, each row containing 14 steps and the swimmers had to compete that twenty times which equated to them climbing over 3640 steps.
} 
hope of satisfying my ferocious appetite. I am just about to walk back to my room when I hear Mum's voice. The sound of it startles me.

Mother: "Carly, what are you doing?"

My back is facing her. My body is frozen. I am worried that she will see that I have gotten a piece of bread out of the fridge. I try to swallow the bread but there is just too much. So, I inconspicuously spit the bread into my hand.

Carly: "I just got a drink of water. Am just on my way to the toilet."

As I walk away from her, I deviate past the bin and carefully throw the piece of bread away.

I breathe a sigh of relief. That was close.

\section{Carly}

"14 years old"

Part 2

As I walk out of the pool with one of my team mates, my stomach gurgles with hunger. I am so hungry that my stomach is actually hurting. Before we walk out of the glass doors at the front of the complex, I notice my team mate feeling around in the bottom of her swimming bag for some coins. Much to her delight, she finds some and heads over to the chocolate machine. She puts the money into the machine and pushes the button. Out the bottom of the machine, a chocolate appears. She reaches back inside her bag and finds some more money.

Candice: "Carly, do you want one? It is my shout."

Maybe I shouldn't.

I don't want to get into trouble.

I don't want to get fat.

Candice is so lucky; she is super skinny and can eat chocolate and get away with it. 
Carly: "Ok, why not. Thanks"

I put the coins into the machine and press the buttons. Out the bottom, my chocolate appears. I reach in and get the chocolate out. We then walk out of the front doors of the complex. Candice goes in a different direction to me. I walk towards where Mum usually parks. She is not there yet. I take the wrapper off the chocolate. Instead of taking a bite, I shove the entire mars bar chocolate into my mouth.

Tastes so good.

My mouth is so full that my jaw hurts. The juices from the sweet chocolate squeeze out as I bite into it. The juices seem to keep coming out of the chocolate, making me choke. I try not to cough as the chocolate will come out of my mouth. It is at that moment that Mum drives in.

Don't want her to catch me.

What should I do?

I run back to the pool and as I pass a garden, out of eye shot from my Mother, I spit the chocolate out. I run inside the pool and straight to the toilets. I go over to the wash basin and get a mouth full of water. I gargle the water. Then I put my fingers inside my mouth and using my finger nails, I scrub the inside of it, removing all residues of the chocolate.

Don't want to get caught. Mum will be able to smell the chocolate on my breath.

I walk out of the toilets and head to the car.

Lucky that I didn't get caught.

\section{Jenny}

"15 years old"

My muscles are weary as I walk from the pool to the food hall for dinner. Have swum 14 kilometres today. It's the first time that I have been a member of the 
Australian Swimming team so I am still getting used to training with such intensity. As I open the door to the food hall, the smell of apple crumble and custard surrounds me. My stomach starts to grumble in anticipation.

I am so hungry!

I fill my plate with lasagne and salad. I walk with my plate to the seating area. My eyes scan in search of some familiar faces.

Have to sit with my friends.

Thank goodness I find some. As I sit down, I notice some of the swimmers at my table are just about finished their dinner.

I wish I was a sprint swimmer; they always get out of the pool early!

As I begin eating, a few get up and head back to their rooms. One female swimmer and one male swimmer stay with me while I finish. They are senior swimmers who have spent extensive time training and living at the Australian Institute of Sport and have been selected in many representative teams. I remember watching them compete on television in awe when I was much younger. Now, I am sitting here with them as their teammate.

I eat everything on my plate but I still feel hungry. I express my thoughts verbally to my teammates.

Jenny: "I am still hungry! I might get a jam sandwich to take back to my room."

Male senior swimmer: "Jenny, a sandwich is 300 calories. Do you think you really need them?"

I have no idea what he is talking about so I just smile. My curiosity gets the better of me.

Jenny: "What are calories?"

Male senior swimmer: "Are you kidding? Calories are the energy value of the food that you eat. Your body only burns a certain amount of calories 
each day. If you eat more than you burn, then you will put on weight. If you put on weight you won't swim fast."

Heck, I ate a doughnut and drank a milkshake while I was at the shops today.

I wonder how many calories I have eaten. Eating guilt is introduced to my consciousness for the first time. Suddenly, I am not so hungry. I head back to my room without the jam sandwich.

(McMahon, 2007).

\section{Jenny \\ "16 years old"}

As we arrive at the pool. It is buzzing with competitors from other countries. I stretch with my teammates. It is like being cast in the movie 'groundhog day' as every day is exactly the same. After stretching, I line up to get weighed in the middle of the concourse in my swimwear. Two of the Australian swimming team managers are standing in front of us with scales systematically weighing us. I feel exposed as a lot of competitors are looking at us. As my turn approaches, panic overcomes me.

What if I have another weight increase?

Two days in a row, my weight has increased by 0.15 of a kilogram. I remember drinking water on the bus on the way to the pool. Anger overwhelms me.

You idiot, that water could affect my weight!

Instead of getting my weight recorded, I inconspicuously jump into the pool and start training. As my body becomes submerged in the water, my thoughts drift to the team managers.

What will their response be with another weight increase?

Hopefully they will forget to weigh me. 
As I pull myself out of the pool at the end of the session, one of the team managers approaches me carrying his clipboard. Without saying a word, he points to my name on the clipboard. There are two weights recorded next to my name, 72.5 kilograms and 72.65 kilograms. Everybody else has three weights next to their names. There is a long silence.

Manager: "Jenny, after you get changed you need to weigh-in."

I cannot find the words to reply. In the showers, I try to calm myself but my thoughts are totally consumed with the weigh-in.

Maybe I have just sweated some of that water out during the session? What happens if that is not enough? Go to the toilet Jenny. That could help.

As I walk outside of the change rooms, the managers are waiting for me carrying their clipboards and scales. My feet step carefully onto the scales. My body is motionless, careful not to bump the scales and cause a slight increase in the number. My weight is 72.85 kilograms, another increase of 0.15 kilogram. The two managers look at each other and are not smiling. The larger of the two starts speaking.

Manager: "Jenny, we are concerned with your weight increases. You need to think about whether you still want a place on the team because your weight is indicating that you are not remaining focused and committed. Your skin fold readings show us that you are losing fitness. We will be interested to see how you go in your skin fold measurements tomorrow?"

I begin questioning my level of commitment.

At dinner that night I hardly eat. I am really worried about my weight. Have to fix my body. My consciousness is alerted to the team managers' gaze. They are looking to see what is on my plate. My teammates are sitting around me. I pile some corn onto my fork and bring it to my mouth, can see them looking, then they whisper to each other. I feel guilty for eating. As the spoonful of broccoli approaches my mouth, they continue to gaze at me.

Their constant gaze suppresses my appetite, creating a false sense of fullness. Somehow my stomach has been tricked into feeling satisfied, however I have only 
eaten three spoonfuls of food. Most of the team decides to gather in one of the rooms to watch a movie but I am desperate to do something about my weight.

Maybe the sauna could help with my weigh-in tomorrow?

Walking into the sauna takes my breath away. It is so hot and uncomfortable. I find it difficult to breathe and become thirsty very quickly.

Don't drink Jenny that could affect your weigh-in tomorrow.

I want to get out of the sauna.

You have to stay, you have no other choice!

At 9pm, I walk back to my room. Feeling really dizzy and short of breath as I climb the stairs. At the top of the stairs, I run into another female swimmer. Her eyes lock with mine but I look away. She can see that something is wrong and asks me if she can help?

Jenny: "My weight has increased two days in a row and I am really worried about it!"

She gives me the biggest smile.

Teammate: "I can help you!"

We walk back to my room together. When we arrive, she grabs my hand and walks me into the bathroom. She tells me to try sticking my fingers down my throat. I watch her as she shoves her 2 longest fingers into her mouth. I wince as she gags.

Teammate: "Jenny, if you bring up your dinner, it will help with your weight in the morning."

As she is standing there, I stick my two longest fingers down my throat. I feel embarrassed as I do it. It hurts and my throat gags. One tiny piece of corn comes up.

Teammate: "Try again!” 
Nothing happens.

Teammate: “Don't worry Jenny, I didn't get it first go either. I do it all the time now though. That way I don't have to worry about eating too much and putting on weight. You need to do it straight after dinner otherwise it won't work."

I am really stressed; still have to get weighed in the morning. Haven't vomited up enough food to make a large enough difference with my weight.

Teammate: "I have another way that can help"

She runs back to her room, reappearing in a matter of minutes. She is carrying a small bottle with purple writing on it.

Teammate: "This will make you go to the toilet and help you lose weight.

They are laxatives. The instructions on the bottle say to only take one or two laxatives. I take seven because it makes you go to the toilet more. You will start going to the toilet in a matter of hours. Jenny, you are desperate. Take seven."

Relief surrounds my body as I climb into bed.

At least my weight will be ok in the morning.

My eyes open. The sun is not up yet. My stomach muscles feel like they are in the spin cycle of a washing machine. Begin to panic. I was supposed to start going to the toilet before now. Have to race today. The heats are on in less than four hours. Can barely move. During the next hour, pain encompasses my entire body. I crawl on my hands and knees to the toilet and pass seven bowel movements. Feel so weak

(McMahon, 2007).

\section{Jenny}

"Another story as a 16 year old"

Ouch! 
My muscles are aching as I walk down the stairs. It is not the familiar training ache but rather an ache from a lack of sleep. Went out last night to celebrate the championships being over. Am so relieved that the competition is finished. Just don't find competition enjoyable, it is such a pressure time, especially trying to achieve the expectations of those around me. Still have one final weigh-in and skin fold test before I can be released from the team and fly home.

I hope that I have not put on weight.

Don't want another lecture from the managers and coaches. Don't understand why we have to do this when we are not even competing anymore. Cannot fathom why they get just as upset if we have put on weight after our races are finished.

Don't want to be looked upon as being uncommitted again.

Arrive at room 168. There is a line up already. Can hear the physiologist calling out numbers. I hear the number 14 called.

Wonder what site that reading was taken from?

Who is getting measured?

Is it one of the female swimmers?

I stand on the tips of my toes and can see that it is one of the girls but cannot make out who it is.

Was it taken from her thigh?

Heck, five days ago my thigh measurement was 21.

My heart rate starts to increase as my body becomes filled with panic as my turn approaches.

As I get to the doorway, I peer inside. I notice that the managers have an efficient system in place. The physiologist is taking the skin fold measurements and one of the team managers is recording the information onto a clipboard. I see the swimmers in front of me who have been measured move to the other side of the room where they then ready themselves to get their weight recorded. A coach is taking the weight readings and the other manager is recording the information. I 
cannot believe how quickly they are getting through the line. The swimmers move so efficiently around the room - robotic like from one measurer to the next. It looks like a factory production line.

As the line gets closer to the front door of the motel room, I notice that a mother of one of the female swimmers is standing inside the doorway listening intently to the swimmers' skin fold measurements. Her face is straining slightly. She is trying to hear the measurements above the noise we are making. She moves herself closer to where the coaches and managers are testing. Surely she isn't listening to our numbers?

Glad that it is not my mother standing there.

As my turn approaches, my stomach muscles start to tighten and my heart rate increases.

Hope that my weight and skin folds have not increased.

I have really tried to eat as little as possible since finishing racing three days ago. I knew that I would have to pass this final test until next trip.

Hope the laxatives and sauna have worked?

I hear my name called. It is my turn. My eyes lock with theirs as I shed my clothes and expose my body.

Hate my body. It is such a failure. Feel vulnerable. Need to fix it.

I turn my mind off so my body is present but my mind is elsewhere. It is like I am floating above. Can see and hear what is happening but have left my-selffor protection. My body surrenders to their pinching and prodding. It is a matter of minutes before the pinching is over and I am alerted to their response. My numbers have increased.

(McMahon, 2007).

\section{Jenny}

"17 years old" 
The scales read 72.6 kilograms. I hop off the scales and gaze into my Mother's disappointed face. Her expression reveals her disappointment in my body. I shift my gaze to my dietician who is sitting opposite me. She is smiling. It is not her usual smile and it seems like she is straining. The room is silent for a moment. My dietician breaks the silence.

Dietician: “Jenny, don't be disheartened. I know you are desperately trying to lose weight. There are reasons why this may not be happening for you. Do you have your periods?"

Jenny: "No"

I sit motionless as she checks my food diary.

Dietician: "You have done really well with your eating this week."

No red circles. She usually circles any food that I have eaten that is forbidden. I see her move her focus to my eating plan. This is a plan that she has devised that informs me what I can eat and when. My body stiffens. I am already struggling with the little food that I am allowed. I hold my breath as she lifts her pen and moves it towards the plan. She moves it towards the dinner section on the bottom of the page.

No, not dinner. I am always starving at dinner. Please don't let it be dinner.

Dietician: "Let's cut out all carbohydrates in the evening for the next week and we will see how that goes. I will see you at the same time next week."

I climb into the car and fasten my seatbelt. I am sitting in the front passenger seat and my Mother is driving. The car is silent. A silence that makes my entire body stiffen with anxiety.

Mother: "Have you been sneaking extra food?"

Jenny: "Mum, I haven't. I really stuck to what she said."

Mother: "What are you eating when I am not around?"

My eyes fill with tears. My body is tense. 
I hate that I can't do this.

Thoughts run through my head. I wonder why I am having so much trouble losing weight. I feel powerless.

Mum drops me back at school. I feel relieved. No more talking about my weight.

I arrive home from training that evening and my mother announces that we are going to the 'Pizza Hut' for dinner. We arrive at the restaurant and as we sit down and as we do, the waitress moves towards our table. She has a notebook and pencil.

Waitress: "What would you like to order tonight?"

My mother orders for my brother, my father and myself. I sit there motionless as my order is decided for me.

Mother: "Three all you can eat pizza and salad bars and one all you can eat salad, please."

I do not have to guess which one I was having. As my mother, father and brother enjoy tasty hot pizza, I walk over to the salad bar. My eyes scan over the choices. There is not much to choose from. I fill my plate with lettuce, tomato, pineapple, cheese and carrot.

I wish there was some meat to go with the salad.

I am so hungry, always am after swimming a 12 kilometre day. As I walk back to the table from the salad bar, my mother's gaze just seems like it is fixated to my plate.

Mother: "You cannot have that cheese."

She grabs her fork and scrapes the cheese from my plate onto hers. I do not reply. A knot appears in my stomach as I eat my salad. It seems like my mother's eyes are watching over my every mouthful. With each mouthful that I eat, the guiltier I become. The salad is tasteless. I find it hard to swallow. It is like something is stuck in my throat. I feel sick, a sickness that I have become all too familiar with at meal times, so I stop eating after only a few mouthfuls. 
(McMahon \& Dinan-Thompson, in press).

\section{Jenny \\ "25 years old"}

Am desperate to lose weight.

The Olympics are my dream and it seems that my body is standing in the way of me achieving this dream. The visits to the dietician are not working and I am sick of my coach commenting on my body and questioning my level of commitment. I have been training hard, so shouldn't that all be that matters to him. Have booked an appointment with my doctor. Am hoping that she can help. I am never going to get the performances that I desire with a body like this.

I arrive at the doctor. As I am sitting in the waiting room, I scan the room. There are three people waiting. Two are elderly and are husband and wife. The other person is a young man sitting next to me. He has a terrible cough. Ifeel uncomfortable sitting next to him as I can feel his warm breath on my face as he coughs. I stand up to walk outside for some fresh air, and as I do, the doctor appears out of her room and calls my name. I walk into her room and sit down in the chair beside her desk.

Doctor: "Hi Jenny, what can I help you with today?"

Jenny: "I think that I have told you before that I am training for the Olympics. Well, I have been really trying to lose weight and I am having trouble. I am not sure if there is something wrong with me as I am training six hours a day and have been sticking to what my dietician has been saying but I am still getting into trouble from my coach for carrying too much weight."

Doctor: "I have your routine blood test results here from last month and everything appears normal except for a low iron level. Your thyroid function is normal so there is nothing that I can see that should be stopping you from losing weight. Sports medicine is not my specialty but there is a sports medicine doctor up the road that could be of more assistance. I will ring him now and try and get you in." 
She picks up the phone and calls the sport medical centre. She talks to their receptionist. She then turns to me.

Doctor: "He has an appointment in two hours. Is that ok with you?

I nod.

Two hours passes and I arrive at the sports medicine surgery and fill out the necessary new patient details. My doctor has sent a copy of my blood tests with me to give to the doctor. It is not long before my name is called. I am surprised by the appearance of the doctor. He is older with greying hair. I assumed that I would be greeted by a fit and athletic looking person. For the second time that day, I sit on the chair beside the doctor's desk.

Sports doctor: "Hi Jenny, what can I help you with today?"

Jenny: "My doctor has sent me along to you because she said that you might be able to be more of assistance to me than she could be. I am a swimmer and I am in training for the Olympics. I am having trouble losing weight. My coach says that I need to lose weight if I am going to be any chance of making the Olympic team. I have been visiting a dietician for ages and have hardly lost any weight. Here is a copy of my recent blood test results which show there is no reason for me to be not losing."

Sports doctor: "Can you jump on the scales for me?"

Sports doctor: "82.3 kilograms. You can take a seat again for me. Do you have any other medical conditions or allergies?"

Jenny: "No."

Sports doctor: "Are you taking any other medication?"

Jenny: "Only the pill."

He pauses momentarily.

Sports doctor: “Ok, I am going to prescribe you some thyroxine. Thyroxine is a medication, which alters your thyroid function. The thyroid function can assist in weight loss. Your function is normal at the moment, but what the 
medication will do is make it overactive and speed things up for you. The speeding up of things will assist you to lose weight. You will need to take one tablet morning and night. Come back and see me in two weeks and we will see how you are going?"

As I walk out, I feel content. The sports doctor has helped me to find the answer. Now, I will lose weight and now I will make the Olympic team.

\section{Jenny}

"Another story as a 25 year old"

It is 5.35am. I walk into the pool and my coach is already there. He calls me over.

Coach: "Jenny, we have the physiologist from Queensland Academy of Sport coming in this morning to take your weight and skin fold measurements. He will be coming in once a fortnight until Olympic trials measuring those who will be competing. It is important that we monitor your weight and your body so you are in the right shape for trials."

My spirits immediately plummet. My mind becomes frantic.

I am going to be the heaviest girl.

What if I weigh more than the guys?

Everyone is going to hear my fat numbers.

I can't believe I am 25 years of age and still worrying about this shit.

The physiologist arrives wearing a maroon uniform and greets my coach like a long lost football buddy. He then readies his equipment. He opens a black rectangular box and pulls out a pair of steel callipers with a gauge attached. My heart beats uncontrollably as I prepare myself to be exposed. I distract myself by beginning my stretching routine, a pre-session ritual. I arch my back like a cat stretching after a long morning nap. I don't want to be the first one tested so I take my time with stretching. I notice the other swimmers that are being weighed, pinched and prodded all have gleaming smiles on their faces. 
Their fat numbers must be good.

I am going to be the only one with bad fat numbers.

I am such a loser.

I should have been doing more running. I shouldn't have eaten dinner last night.

Physiologist: “3."

Male teammates: "Wow, you are less than us on our biceps."

I then see the physiologist adding up her scores.

Physiologist: "61 overall, great work."

None of my measurements are ever under a reading of ten. I can't believe that she just got 61 overall. I know that I am going to be well over 70. I am going to get into so much trouble from my coach. Need to fix my fat numbers. Need to fix my body.

Physiologist: “Jenny, you are up.”

It is my turn. I slip my tracksuit pants off and lift my shirt up over my head and drop it on top of my bag. I feel naked in my togs and try and cover my body with my arms inconspicuously. I walk over to the scales and step on. My weight is recorded. I sneakily glance out of the corner of my eye to see if anyone is listening. I step off the scales and the physiologist picks up the steel calipers in readiness. He creates a black mark on my bicep. He then grabs the skin on bicep in a pinch like action and he places the jaws of the steel calipers onto my pinch of skin. The needle in the gauge moves I try to not move, as I am worried any slight movement will move the needle in the gauge in the wrong direction.

Physiologist: "14"

I am such a failure. 
At the end of the pinching and prodding, I notice the physiologist quickly calculating the total of my fat numbers. It is only a matter of moments before he announces the result.

Physiologist: " 88 "

A total over 70, I know what this means.

(McMahon \& Dinan-Thompson, in press).

\section{Jenny}

"Another story as a 25 year old"

Olympics are only 12 months away. 15 months have passed since I have been back in the water.

Have to swim faster. Need to swim faster.

My grip on Sydney Olympics is loosening. Even though I am looking fitter than I have done in years, I am still being constantly reminded by my coach and others that I can lose a few more kilograms. Those few kilograms are my nemesis. It is still the one thing stopping me from achieving my lifetime goal of Olympics representation. No matter how hard I work, they are still there, torturing me in such an unkind way, constantly in my thoughts particularly making regular visits to me at meal times. I visualise being on the medal dais. I am receiving my Commonwealth Games silver medal. The Australian flag is being raised.

I want that feeling again.

Need to fix my body!

Swimming fast now but if I lose weight, I will swim faster!

Am desperate! Willing to do anything to lose weight.

Eating healthy has not worked. Dietician did not work. Thyroxine from the sports' medicine doctor to make my thyroid overactive did not work. I feel low. I am such a failure. 
Why are you letting me down this way?

Need to find another answer.

Need to fix my body!

A friend of mine told me that she uses this drug called duromine to lose weight but you need a doctor's prescription to get it. She said that she lost seven kilograms in two months. Have booked in to the 24-hour medical centre this evening after I finish training to get it.

I arrive at the doctor's surgery. An uncomfortable familiarity overcomes me. I sit in the waiting room and my body is rigid. I can't help but notice the other four people who are waiting are either coughing or blowing their noses. The waiting room is such a contrast to my life where everybody is fit and healthy. The doctor calls my name and I go in and sit on a chair that is beside his table.

Doctor: "What can I do for you this evening?"

Jenny: "I would like to get a script for duromine please."

Doctor: "Duromine is an appetite suppressant and is usually only prescribed for obese patients trying to lose weight. It has very harsh side effects. You don't need to lose any weight."

Jenny: "But I do need to lose weight. I am a swimmer training for the Olympics. My coach is constantly on my back to lose weight. If I don't lose weight, I won't make the Olympics. I have been going to a dietician for ages and I am not losing enough weight. I am desperate. Olympics are only 12 months away."

Doctor: "I am reluctant to do this but I can see that you are desperate. You take one tablet in the morning. I will also prescribe you some sleeping tablets, which you may need as the appetite suppressants can keep you awake. You just take one tablet half an hour prior to going to bed and it will help you go to sleep."

I leave the doctors surgery relieved and happy. 
I have found an answer. Everything will be ok now.

(McMahon \& Dinan-Thompson, in press).

\author{
Jenny \\ “Almost 26"
}

\title{
Part 1
}

It is 4.20am. I wake to the alarm ringing. I climb out of bed and wander half asleep to the bathroom. I climb onto the scales. I am another kilogram lighter. Duromine is working so well, I can't believe it. I have been taking it for a month and I have now lost six kilograms.

Have to lose more.

I grab my toothbrush and look into the mirror. I turn away; I cannot look at myself.

I am ashamed of what I look like and I am ashamed that I have not yet achieved a swimmer body that will bring me Olympic success.

I drive to the pool. I am not excited like I used to be. I now see training as a necessity, a necessity to burn calories. It is Wednesday morning so we have a heart rate. Heart rate sets are intense and hurt a lot. I finish the warm up and feel as though I have swum nine kilometres. I am exhausted already. I commence the heart rate set with the rest of the squad. I have to do $20 x 100$ 's of freestyle on the two minutes. I touch the wall after the first one. My coach yells out my times.

Coach: “1.07."

I grab the heart rate monitor and put it onto my chest.

Coach: "186."

It is going to be a long morning. I have only swum $1 \times 100$ and my heart rate is already at its maximum. I am just not swimming the times that I was four months ago. 
At least my weight loss is going well.

I swim seven kilometres. Time for the gym. I have a long shower before hopping into my gym clothes. I feel shaky as I put on my joggers. I walk to the gym after training as it is just up the road from the pool. It is 9am. I hop onto the exercise bike first. I adjust the settings and put on my walkman. My keenness in the cardio studio has grown. It has surpassed my enthusiasm for training in the pool. Love riding and running as it burns more calories than swimming.

Have to burn 1000 calories!

I ride the bike for an hour but the machine says I have only burned 350 calories.

That is not enough!

I get off the bike and get onto the treadmill. I start running. I adjust the setting so that I am running at 12 kilometres an hour. I burn 650 calories during the next hour. I get to the 12-kilometre mark and I decide it is not enough. I increase my goal; I now want to burn 1500 calories. I run for another 45 minutes.

I look down at the monitor. Sweat is dripping off me. I have burnt 1500 calories and have run 20 kilometres.

Lucky this afternoon is a recovery swim session!

(McMahon \& Dinan-Thompson, in press).

\section{Jenny \\ “Almost 26"}

\section{Part 2}

Coach: “Jenny, you don't need to lose any more weight. You have done a good job but that is enough!"

Those words I have longed to hear from my coach but they just bounce inside of me and just as quickly bounce out.

I can lose more weight. I will show him! I have the power now. 
Have been getting so many comments about the way that I am looking, Ifeel accepted finally. I have proven that I can stop eating and lose weight.

Want to lose more.

My descending weight on the scales and the positive comments has become a drug.

Have to keep going!

I get home and it is $8.15 \mathrm{pm}$. I reach into the pantry and find two familiar feeling containers of tablets. I open the lids of both bottles. The first bottle has laxatives and they are maroon in colour. I tip the bottle into my hand until I can feel their smooth texture in my palm. I count seven tablets. I tip the bottle again until I have ten. I screw the lid back on and open the lid of the other one. The lid clicks as I twist it. It has a child lock on it. These tablets are not as shiny in colour; they are pale orange and have a chalky texture. I tip three tablets into my palm. I cannot wait to feel my body succumb to its effects and negate the effects of the speed that I took this morning. They are cerepax. I don't take the tablets in any particular order. I swallow the 13 tablets with two mouthfuls of water. I turn on the television, as ER is about to start. Within half an hour, I start to feel the effects of the cerepax. My body relaxes and my mind starts to switch off. I look forward to this feeling and this time of the day. I feel so out of control when I am taking duromine. It speeds my body up and my mind races. I often have delusional thoughts. They are worth it though because they stop me from eating. The cerepax is able to bring me down off the high and gives my body a sense of calm. Without it I would not be able to sleep. The sound of the phone ringing brings me out of my trance. I answer it and it is a male friend of mine that I have been spending some time with. I am glad to hear from him as I really like talking to him. He notices the slurring of my words. I tell him that I am just really tired.

The next thing I realize is when my body jolts awake to a familiar ringing sound. It is my alarm clock. I look at the clock. It is 4.20am. I am groggy and disorientated.

How did I get to bed? 
The last thing I remember was talking to my friend last night.

How long did we talk for?

What did I say?

(McMahon, 2007).

\section{Mandy}

"15 years old"

\section{Part 1}

I stand onto the scales and look down. The three coaches who are surrounding me look at each other and then start to speak.

Coach: "55 kilograms. You have put on two kilograms."

My heart sinks. I cannot understand why I would have a weight increase. I have been so careful, just can't understand it.

Coaches: "you have put on weight. For the next three days, you will eat every meal at the same table as us. You are only to eat fruit and salad. Do you understand?"

I nod and feel intimidated standing there by myself. My own coach is on the team but he is not one of the three coaches standing in front of me talking to me. Ifeel alone. I think the conversation is over but then they start to speak again.

Coaches: "If you are going to continue putting on weight, you are going to have to change your eating habits to keep your weight down."

I become infuriated. I know that I should not respond but I cannot help myself.

Mandy: "Look around you guys. Do you see many other swimmers here ranked number one in the world? Go for it, just do it!"

I can see that they are surprised by my response and they appear to back off. 
That morning at breakfast, I do as I have been told. I get a plate and fill it with fruit. Instead of sitting with my team mates, I sit with the three coaches. Out of the corner of my eye, I notice the eyes of my team mates looking at me. I can see them whispering, wondering why I have to sit with the coaches. I then turn my head and look at them. Everyone has a plate full of the pancakes with delicious extras, making the most of the delicious cuisine. I become angry and frustrated.

For the next three days, I continue with my punishment. I wonder why I am being punished for so long for a two kilogram weight increase.

As a method of self-protection, I withdraw into myself, ignoring my team mates becoming insular. Despite my efforts of ignoring my surrounds at meal times, it is impossible to ignore the coaches who are sitting directly in front of me. As they sit in front of me at lunch on the fourth day, I notice how they stuff one hot dog after another into their mouths. As they finish the pile of food on their plates, they return to the food bar, filling their plates again with the delicious choices. I pick up a piece of pineapple and take it to my mouth. The eyes of the coaches fixate on me, watching me as I put the fruit into my mouth. I feel self-conscious and stop eating. I am starving, so hungry after the heavy training we have done today. I have swum two sessions today, like every other day, churning up the pool for nine kilometres each session yet I continue to deprive my body of the food and energy it is desperately craving because I am being punished.

After I finish dinner, I head back to my room with a senior female swimmer.

Senior female swimmer: "Why aren't you allowed to eat with the other swimmers?"

Mandy: "I have put on weight and the coaches want me to sit with them so they can see what I am eating. I am only allowed to eat fruit and vegetables."

Senior female swimmer: "Come up to my room, I will show you how I have learned to maintain my weight."

I enter her room. We are on our own. 
Senior female swimmer: "After each meal, I just stick my fingers down my throat and vomit. It is a way that I can eat and still maintain my weight."

She takes me into the bathroom and shows me how to do it. Her dinner comes up.

Have to do something - can't let it let me down anymore.

I then model her every move. I stick my fingers down my throat and as I do I think about the body that I need to have, a body that will be accepted by the coaches. My dinner comes up. The fruit burns as it comes up.

This is going to fix everything. This will help me control my body - my weight.

(McMahon et al., in press).

\author{
Mandy \\ "15 years old"
}

\title{
Part 2
}

I wake up, not to the usual way of the alarm ringing but because my ears are hurting. They are throbbing, aching and I cannot sleep. I try to get a comfortable position on the pillow. I can't lie on my side, because the pressure on my ears is just too painful, both are really hurting. So, I lie directly on my back. It is 3 am.

Too early to get up and get help.

It sounds like my heart is inside ears as a loud throbbing beat pound rhythmically. Each time the beat sounds, it throbs with pain. I watch the minutes tick over on my clock, hoping and willing the time to pass quicker so I can get up.

At 5am, I cannot stand it anymore, so I get up and get ready for the pool. I am the first swimmer there but not the first person. The coaches are already there setting up. I approach them and tell them that I am in awful pain. As I am telling them, I can tell by their expression that they are concerned. At first I think they are concerned for me but I soon realise that I am wrong. 
Coaches: “We better get you away from the other swimmers. Don't want them to get sick. You are not to associate with any of the other swimmers, don't eat with them, don't go into their rooms. Do you understand?"

I nod as tears start to well in my eyes. I let out a scream, not because of their lack of sympathy but because of the terrible stabbing pain in my ears. One of the coaches quickly walks me back to my room before any of the other swimmers arrive and tells me to stay in my room until he returns. It is 3.5 hours before he returns.

Coach: "I have booked you in for an appointment at the medical centre. I am going to take you now."

I nod and follow like an obedient puppy. At the medical centre, I explain how I am feeling to the doctor. I cry as I tell him because the pain is just too much. He looks into my ears with his special magnifying instrument. As he touches my ear to look inside, I let out a scream. He shakes his head.

Doctor: "you have massive infection in both ears. There are large puss filled nodules in your ear canals. You need to keep your ears dry for at least four days and under no circumstance are you allowed to fly."

Coach: "But we are leaving in 2 days, we cannot leave her here."

Doctor: "Under no circumstances must she fly. It will be just too painful and her ear drums may rupture because of the pressure. It could cause irreversible damage."

The coach nods and thanks the doctor for his assistance. We walk back to our accommodation.

Mandy: "Who will look after me when you leave?"

Coach: "Nobody, because you are coming with us."

Mandy: "But, I am not allowed to fly."

My comments go unheard. I realize now that I will have to fly, just hoping that my ears will be much better by then. 
At the airport, as we prepare to fly out to the Commonwealth Games, I worry about the flight wondering whether my ears will handle it. They have improved since the other day but they are still so painful.

I try to get comfortable in my seat as the plane taxies out of the airport in preparation for takeoff. The further it taxies down the runway, the more my heart rate increases with fear.

Am so worried about my ears. Hope they don't hurt.

The plane takes off into the air, gaining altitude quickly. The more altitude the plane gains, the more blocked my ears become. It is not long before I cannot hear anything. After about 10 minutes of ascending, the build-up of pressure becomes too much and my ears begin to really hurt. Tears start to trickle down my face. I try to hide my pain but I can't. It becomes too much. I let out a scream. One of the coaches comes over to me.

It is hurting so much.

He doesn't say anything and just goes back to his seat. The rest of the flight becomes a painful semi conscious blur as I am unable to relieve the pressure in my ears.

\section{Mandy}

"15 years old"

\section{Part 3}

We arrive at the Commonwealth Games village where all of the national teams are residing for the duration of the competition. It is a flurry of excitement as the competitors wander the large compound in their national colours. The competitors are buzzing with enthusiasm for the two weeks that lay ahead. Upon arrival at the village, I am whisked away by one of the coaches to have my sore ears examined by the Australian team doctor. The team doctor asks me how long I have had the ear infection for and then he pulls my ear, looking inside. It really hurts. The pain shoots down my arm. 
Doctor: "both ears are extremely inflamed. You should not have flown in this condition. There are puss filled nodules in your ear canal that have recently burst. You said that you had these checked before you left? Did the doctor not explain the risks associated with flying?"

I do not respond, nor does the coach but I know exactly what that doctor said. At the end of the appointment, I head back to my room. The coach speaks to me on the way.

Coach: "Mandy, you still need to remain in isolation. Can't risk anybody else getting this."

Mandy: "But, it is just an ear infection. You heard the doctor."

Coach: "We need to ensure that the other swimmers remain healthy so you are not to eat with the team, train with the team, socialise with them. Do you understand? You better not be putting on weight either. I will come to your room later to check."

The thought of a weigh-in shocks me. It was not what I was expecting. I assumed that because I had not been in the water and had been ill that I would not have to get weighed. I realise that there is going to be no changing of his mind. I sit in my room for the remainder of the afternoon on my own as I have been told to do.

Ifeel isolated.

At dinner time, I walk up to the food hall on my own.

Am feeling incredibly lonely.

I have no teammates to sit with but an Australian team member from another sport invites me to eat dinner with him. This is the first of many meals that I have with him. I welcome the social interaction with him. It is a nice feeling to be able talk to someone again.

After dinner, one of the coaches comes to my room for a weigh-in. He places the scales on my bedroom floor. I don't want to show him my fear-my trepidation in being weighed. So, I walk straight towards them, purposive in manner. Even though I have been very careful with what I have been eating, I still remain 
concerned for the outcome. I hop onto them and immediately look down. It reads 55 kilograms. I have put on two kilograms and am the same weight as I was at the beginning of the training camp. I feel demoralised. The coach leaves my room without saying anything. I immediately cast my thoughts back to a conversation that I had with one of my female teammates on the plane here.

Mandy: "I am not allowed to swim, not allowed to socialise with you, not allowed to eat with you. Am not allowed to leave my room - am not allowed to do anything. I am also worried about my weight with every session that I am out of the water."

Other female swimmer: "Hey, all you have to do is put your fingers down your throat. Everything will be ok then."

I know what I need to do. The conversation reminds me of what is necessarywhat I must do. I walk into the bathroom and shut the door behind me. Tears are streaming down my face. I ready my index and middle finger before plunging them down my throat. 
ACT 2

TALKING SWIMMER BODIES: EXPOSURE TO A PERFORMANCE FOCUSED CULTURE? 


\section{ACT TWO - Prequel}

Prologue: The stories told by Carly occurred when she was between ten and fourteen years of age. She is a club swimmer who has had success at a local and state level. My [McMahon] stories of experience are told between the ages of 15 and 18 - as an Australian swimming representative. Mandy's stories are of experiences that have also occurred within the elite Australian swimming context and culture.

\section{Carly}

"11 years old"

I wrap one arm around the lane rope and fix my eyes onto my coach. I can hear the breathing sounds of my team mates around me. Their panting starts to slow from rapid to intermittent. It is like we are all well oiled machines and our quick recovery represents our extreme fitness. We all wait in readiness for the next training set, focused on the coach, listening in anticipation to what we will be doing. He asks us to divide up the lanes according to our form stroke - our main stroke. I move into lane eight with the other butterfly swimmers.

Coach: "Right, you guys have 20 x 50's jump outs ${ }^{17}$ butterfly on 60 seconds. I want you to hold times that are 3 seconds off race pace."

Three seconds off race pace, my best is 31 so I need to be holding 34 seconds for each 50 metres. As I dive into the water, I feel strong. I know I will be able to breeze through this set. I usually can tell within the first few strokes how I am feeling. Today, I feel strong and fast. I touch the wall at the first 50.

Coach: "Carly, 35."

35 seconds. I am sure I am swimming faster than that. It feels stronger and faster than a 35. I lift myself out of the pool. By this time, I only get 10 seconds break

\footnotetext{
${ }^{17}$ Jump outs are when the swimmer is required to climb out of the pool at the end of each lap. Swimmers are not to use the ladder to assist them in getting out and this has to be done in the specified time frame. Each lap subsequently commences with a dive start.
} 
before I have to dive in for the next 50. I still feel good. When I touch the wall after the second 50, I lift my head out of the water to hear my time.

Coach: "Carly, 35. Carly, if you don't hold 34's, I am going to make you repeat this set at the end of the session."

I start to become panicked. I don't want to have to repeat this set. This time when I dive in, I work my streamline more. Instead of working on my rhythm, I sprint the lap.

Need to make 34 seconds.

I touch the wall on the third 50.

Coach: "Carly, 34."

I finally make the time. It is so hard though. It was 100 percent effort. I thought that I was feeling strong. I thought that I was swimming well but I was wrong. I slug my way through the next ten laps. I just make the times but my body starts to become fatigued. I find myself questioning my fitness. I should not be hurting this much for these times. On the fourteenth lap, I touch the wall and lift my head to hear my time.

Coach: "Carly, 35. You are making this set up at the end of the session."

Ifeel deflated, demoralised.

Doesn't he know that I am putting in everything I can?

I am disappointed in my times and myself but I know I am putting everything into this set. At least by repeating this set after everyone is out of the water, I am putting extra money in the bank, putting in extra than my competitors would be which will in turn make me swim faster. However, I feel somehow hard done by as I realise, that half of the other swimmers aren't making their times either but I am the only one that has to stay back and repeat it. I wonder why I am the only one that always has to stay back and repeat sets.

It isn't fair. 
I feel singled out and picked on. I am presented with a quandary between being hard done by and knowing that this is actually good for me so I say nothing to my coach and accept it obligingly. I continue to battle my internal thoughts which are presenting me with such contrasting feelings.

\section{I hate Tom.}

But I know this is good for me.

It is 6.30pm. The rest of the squad gets out of the pool but I am not allowed as I have to repeat the set. I don't know how I am going to do 34 's now at the end of an eight kilometre session when I couldn't do it earlier. My body feels fatigued from the long day.

Carly: "Why am I the only one that has to stay in? Half the other guys didn't make their times!"

Coach: "You better get on with it otherwise we are going to be here all night. Champions don't complain."

I dive in for the first 50. I am so angry and start to cry. I cry really hard as I throw my arms over in the butterfly motion. I touch the wall after the first lap and lift my head to hear my time.

Coach: "34. Right, just need to do another 19 at that pace."

At the end of the second 50, I notice that my Mum has arrived to take my brother and I home. I can tell by the look on her face that she has realised that I am staying in because I have not done what my coach has asked me to do. I complete the next five laps. My times get slower and slower. I started off with a 34 but I am just so exhausted now and am unable to hold that pace.

Coach: "You are dropping off, get a move on."

I climb out of the pool and instead of diving in for the seventh 50 metre, I sit on the side. I start to sob. I can see that my coach has noticed that I have stopped swimming but he does not come up to me. Instead he leaves me sitting on the side of the pool crying. I sit there for a long time. My body is now shivering from the 
cold, from sitting for so long. I notice that the time is 7pm, which means I have been sitting on the side for nearly half an hour. Finally my coach comes up to me.

Coach: "You can get out, but I want to see you make 4 x 50's under 35 seconds. If you don't make them, you can start the 20 over again from the beginning."

I cry but my tears have no effect on him. I get up; realizing that there is no way that he is going to let me go home until I have done them. Everyone has left now, except for my mother and my brother who are still sitting in the grandstand and another coach from another club who coaches on the other side of the pool. I slog through the next 4 laps, doing what my coach has asked. My body sobs as I throw my arms over. It is hard going but anger gets me through it. I make the times but it is now 7.15pm before I am finished. As I pull my weary body out of the water, my coach starts talking.

Coach: "You should have done that the first time. Champions don't slack off!"

Carly: "But Tom, I felt as though I was swimming much faster. I was putting everything into it!"

I walk over to the club room and put my pull buoy, kick board and flippers away. As I do this, the other coach from the other club who trains at the same time as us on the opposite side of the pool starts talking to me.

Other coach: "You know Carly, Tom wasn't calling out the right times for you. I timed you with my stop watch and you were holding 32 's!'

Carly: "Are you kidding?"

Other coach: "I thought that I might have got it wrong so I timed a few of your laps because you were looking so strong and they were all 32's."

I head to the showers trying to make meaning of what I have just heard. I can't believe it. How could Tom do such a thing? 


\section{Carly}

"Another story as an 11 year old"

I am so excited about getting back to school today. The Easter holidays, for me, were filled with the usual training but I really missed my friends. As I walk in through the sliding doors of our grade six classroom, I can see Miss Hogan who is busily working, organising for the day. Some other students are helping her. I walk over to see what they are doing.

Carly: "Hi, what is going on?"

Miss Hogan: "I am just organising a buddy class for us!"

Carly: "What is a buddy class?"

Miss Hogan: "It is another grade 6 class from a school which is 270

kilometres northwest of our school. Students from that class will be conversing with our class. I will even be allocating you a student from that class who will be your very own pen friend, someone you can write to, talk with. In four weeks time, our class will actually be travelling to their school and you will have the opportunity to spend three nights in your pen friend's home! 4 weeks after we visit them, they will be coming here."

Carly: "Wow, that is so exciting. I cannot wait! It is going to be so much fun!"

Later in the day, Miss Hogan allocates us each a pen friend. She gives every student a card with a photo of our pen friend on it and their contact details. I can hardly contain my excitement. I cannot wait to get home and write my pen friendSarah a letter.

The weeks pass quickly and during that time I write regularly to my pen pal, Sarah. Over the time, we converse often and it is not long before Sarah has become a really good friend, someone who I can confide in-someone who is not involved in swimming. In the last letter that I got from Sarah, she told me how excited she was about our class visiting her school next week. I can't wait either. It is going to be awesome. 


\section{Can't wait to meet Sarah!}

I am counting down the sleeps. It is four to go until we leave, just four until I can see Sarah. The school trip has been the best distraction for me, a distraction from the mundane heavy workload that I have been doing in the pool. My coach has been giving me lots of miles at the moment, preparing me for the Pacific School Games. The competition is only four and a half weeks away. That night as I am helping Mum set the table for dinner. I find myself thinking about Sarah and the fun that we will be having in just a few days.

Carly: "Mum, do you think it will be cold where Sarah lives?"

Mum: "Actually Carly, that is something that I have been meaning to talk to you about. We have decided that it is not a good idea that you go on the trip with your class, so we think that it will be best if you stay home. It is just too close to the Pan Pacific School Games and we don't want to disrupt your training at the moment. It could affect everything that you have worked so hard for."

Carly: "Mum, it is only a three night trip. I was speaking to Sarah and there is a pool out there. If I can get to the pool for a couple of sessions - that will be enough."

Mum: "We have already decided. Sorry Carly."

I am shattered. I walk to my room and throw myself onto the bed, burying my face into the pillow. I scream!

How could they do this to me!!

I come out of my room.

Carly: "Mum, is it still ok that Sarah stays with us when she comes to our school in four weeks?"

Mum: “Carly, I just don't think it will be a good idea. That is only a few days before you leave for the Pan Pacific School Games. You really need to be focused that week. I will organise with Miss Hogan to make sure Sarah has got somewhere else to stay. 
I am devastated.

Why can't I be normal?

Why can't I have friends?

I hate my life!

\section{Carly}

"Almost 12 years old"

I let the hot water from the shower pour all over my body. It is so warm but my body is still shaking. I am still cold from that freezing water. Cannot believe the organisers would hold national age group championships in an outdoor pool in April. The conditions are so different to what I am used to, and as a consequence I feel like it is affecting my performance. I am not swimming well. My performance this morning in the heats was disappointing not unlike my other performances so far in this meet. My times were well outside my best and I still have not medalled. Standing in the shower, I try to find reasons for my poor performance.

\section{Can't understand why?}

I train so hard, put in so much all the time, sacrifice so much, yet it seems that I am getting nothing in return.

I feel safe in the shower. I have the bathroom door closed. Mum is sitting on the bed watching television. Even though there is only a door that separates us, I welcome the solitude. 20 minutes passes by and I am still standing under the warm water trying to get warm. There is a knock at the door. It is Mum!

Mum: "Carly, are you ok?"

Carly: "Yep, just trying to get warm."

I am apprehensive about getting out.

Want to stay in the shower.

Sick of getting lectures from Mum. 
Just wish she would get over it!

I feel bad enough how it is, just don't need Mum telling me that I need to do this and I need to do that, I need to train harder, I need to warm up properly. I have been competing for a long time now. I consider myself to be quite experienced and I don't need to be told what to do when I am doing it all anyway.

I finally climb out of the shower. My skin feels warm now. My muscles have also loosened up after being so tight from the cold. I dry my skin with the fluffy motel towel and put my flannelette pyjamas on. As I open the bathroom door, I see Mum sitting on her bed watching television. She does not look happy. Her look, a precursor to a long lecture, makes me anxious.

Carly: "Mum, I am really tired. I am going to sleep."

Mum: "I think we need to talk about this morning."

Carly: "Can we do it later?"

I climb into bed. My body welcomes the warmth and the comfort. I close my eyes and slow my breathing down, relaxing my body. I am not really tired but am trying to escape another lecture. There is a partition separating my bed and the bed that my Mother is in. Even though it is not very high, it gives me a bit of privacy. It would only be five minutes that I have been lying in bed for when I hear Mum moving in her bed. It sounds like she is getting out. Ifeel the shadow from her body near my face. She is looking over the partition. I pretend to be asleep as I don't want the lecture. After a few seconds, she gets back into bed. I then hear her start to cry. She tries to muffle her sounds, but she is definitely crying. It goes on for a couple of minutes.

Why is she so upset?

It suddenly dawns on me that she is actually crying because of me. She is crying because of my performances.

Why can't she leave me alone and just let me swim?

If she did not lecture me all the time, criticizing my every fault, then maybe I would actually swim faster. I'm doing the best I can. It is not as though I enjoy 
swimming like crap. I think about my performances and my mother's reaction. I become fearful of what is happening to me and my ability to swim fast.

Everyone's going to start beating me all the time soon.

Haven't swum well since going to that training camp last year where I got fat.

I am letting everyone down.

I hate my life.

Just want to escape.

\section{Carly}

"12 years old"

My performances today at the State titles are no different to my performances of late. I am swimming so badly.

I am over it.

I am totally frustrated. As I am lying in bed - the top bunk of my brothers bed where it is a bit cooler having a lunchtime rest between the heats and finals session, Ifeel depressed, disillusioned, with my life and my swimming.

\section{I hate my life!}

I hate myself!

I keep my eyes closed even though I am not asleep. I cannot bear to open them for fear of Mum lecturing me. Had another lecture from her this morning. The words are still ringing in my ears; I try to block it out.

Am so over the lectures!

I wish Mum would just leave me alone.

As I lie in my bed, I think about the finals swims that I have tonight. 
I don't want to do them!

I am ashamed of the way I am swimming. I just don't want to go through it anymore and most of all; I don't want the lecture from Mum.

Maybe I can get out of it?

My mind becomes frantic, thinking of ways that I can get out of swimming tonight.

If I say I have the flu, it won't be enough. Mum will still make me swim.

Then it suddenly dawns on me:

If I break a bone, then I won't have to swim!

I then think of ways that I could break a bone, making it look like an accident to get me out of competing for the rest of the competition.

Collarbone would work!

If I fall out of my bed, rolling the wrong way, I could fall and break my collarbone.

Ifound it. I have the answer!

I place my body on the very edge of the bed, readying myself to fall out. I start to roll out but panic and stop myself. I get angry for not doing it. I run through my options again as I am desperate to do something. I can either swim or get out of it with a broken bone. Neither seems to be good options. I start to think about what my mum's reaction would actually be if I did break a bone and realize that Mum would make me swim anyway. She has told me so many times in the past how she finished her netball games injured. Once she had a broken collar bone and the other time, a girl's tooth had broken off in her nose. I don't have the guts to break my bone and I probably would not break anything anyway and the whole thing would be a waste of time.

Probably won't even make the state team this year. ${ }^{18}$

\footnotetext{
${ }^{18}$ It is worth mentioning that as Carly was recalling this story of experience, she mentioned in her words; "I did make the state team though, didn't swim as badly as I thought as I still won races
} 


\section{Carly}

"13 years old"

As I sit in the doctor's surgery, I recall my coach's words:

Coach: "You need to go the sports medicine doctor. I will give you his number so your parents can make an appointment. He will test your blood and give you some supplements to take. We need to make sure that you are in the right shape to swim fast. Most of the senior swimmers see him. He will help you swim faster."

I latch on to the latter part of what he says; "he is going to help me swim even faster." Wow, this is great. Somebody who can help me swim faster by testing my blood and giving me tablets. A doctor appears from the room and calls my name. I walk towards the room that he has just appeared from. My mother is with me.

Doctor: "Hi Carly. So, you train with Tom?"

I nod and my mother speaks for me.

Mother: "Yes, she is in training for the Olympics next year. Tom mentioned that a few of the other swimmers see you. We want to give Carly every possible advantage and we were told that you can help."

Doctor: "Well, let's start off by taking some bloods."

The doctor picks up the phone and asks a nurse to come into his room and take my blood. It is only a matter of minutes before a woman appears in the room carrying a plastic dish. She puts a tight thing on my arm and cleans the inside of my elbow.

Nurse: "You are going to feel a small prick."

I close my eyes and think about swimming fast. It is only a matter of minutes before it is all over. The doctor starts to speak.

and broke records, just was putting too much pressure on myself and had way too much pressure placed on me by others [coach, mother]." 
Doctor: "the results will take a couple of days to come back. If we make an appointment for Friday, you can come back and I will have some medications for you to take."

I feel excited as I leave the appointment. It seems so simple, so easy - taking a few medications and I will swim faster. Such a contrast to the daily grind I put my body through in the pool. I cannot wait for Friday to come so I can start taking the tablets.

The three days pass slowly as I complete the same mundane training that I usually do. After the morning session, Mum takes me straight to the doctor. When we arrive, the receptionist greets us.

Receptionist: "The doctor has been called out for an emergency but he left these for you."

She reaches down under her desk and pulls up a large plastic bag full of different coloured tablets. Each colour is separated in a plastic cylindrical type container. My eyes nearly pop out of my head.

Carly: "Are they all mine?"

Receptionist: "They sure are. The instructions are on each container. You will need to take some once a day and some twice a day."

My mother hands her some money and we walk out with the bag full of tablets.

\section{Carly}

$$
\text { "13 years old - nearly 14" }
$$

State titles are over and I am looking forward to school going back. I miss my friends. School is such a break from my swimming life. It is a place where I can get some reprieve from swimming. I find my school bag in the bag of my wardrobe. I pull it out and notice that my school lunch is still in there from last year and for the six weeks that it has been sitting in the back of my wardrobe, it has grown some lovely green colours on it. I take it outside and throw my half eaten lunch in the bin and start to wipe it out. Mum walks into the kitchen and can see what I am doing but asks anyway. 
Mum: "Carly, what are you doing?"

Carly: "Cleaning out my school bag. School goes back next week."

Mum: "Oh, that is something that I have been meaning to talk to you about. You are not going back to school. You are going to be home schooled."

Carly: "WHAT?"

Mum: "Olympic trials are in five months and you need no distractions. You need to be putting in extra training and resting through the day. School will just hinder your training. You will still have some school work to do but you can do it after you have had a nap."

Carly: "But, what about my friends?"

Mum: "You can catch up with them when you get back from the Olympics."

I walk into my room and throw my school bag onto my bed. I am angry.

Who made this decision - Tom or my parents?

Why didn't anyone talk to me!

I bury my face into my pillow and cry.

\section{Carly}

"14 years old"

\section{Part 1}

The referee blows his whistle signalling the eight of us to stand onto the start blocks in preparation for the race. It is the final of the 100 metres butterfly. This is it. It is everything that I have trained for. It feels like minutes as I stand there on the starting block with my fellow seven competitors. I think about my dream - an Olympic gold medal. For me, it is the ultimate, the pinnacle and it is all resting on this one race, the 100 metres butterfly. If I don't finish first or second, my dream will be over, everything that I have worked for, from seven years of age will be wasted. This is my chance to make it, to be selected on the Australian Olympic 
team. I am tense but focused. I think about all of the work that I have done in the pool for the past seven years, two sessions per day, over 80 kilometres of swimming per week, weights sessions, stairs, runs, many nights of going to bed starving striving for the ultimate champion swimmer body, regular weigh-ins and skin-fold tests.

I am in the hands of the starter. The gun goes off and I dive into the water. My body takes over. I know what needs to be done. I don't feel strong but I try not to let it panic me and instead I focus on my technique. Before I know it, I am approaching the 50 metre mark and I notice that there are several girls ahead of me. I still try not to panic and focus my entire attention on the second half of the race. I know I have done more training than any of the girls racing against me and I intend to draw on it and come home like a steam train. I accelerate through my kick and the push in my stroke. It is short lived and don't have the strength to continue it for long. Only 10 metres to go, I finish it off but I know that my dream is over. I touch the wall in sixth position. I will not be going to the Olympics. I am devastated. Am not sure if I am actually dreaming this or not? It all seems surreal, in slow motion, as if I am watching my life through a movie screen.

I climb out of the pool and grab my towel. I walk over to the diving pool and dive in. As I am warming down, throwing my arms over, I try to make sense of what just happened. I cry. So many questions run through my mind.

What just happened?

Why was I so far outside of my personal best?

How can I work so hard for so many years and then not make it?

What will I do now?

What will Tom say?

I climb out of the diving pool and put my tracksuit on. I head back up to the grandstand where I know my coach will be waiting for me. I take my time as I am still trying to make sense of my poor form. My eyes scan to where my club is sitting. I walk towards them. One of the senior female swimmers in my club has just spoken to Tom and is heading down for her race. Her eyes connect with mine 
and our shoulders nearly rub as she walks past me but she says nothing. Her face is expressionless. This is not out of character for her as she has ignored me in the past when I have not swum well. She tends to behave in the opposite when I am swimming well. I walk towards my coach, he is only metres away. His eyes connect with mine. I stop when I get a metre away from him. He pauses, looking at me. He then raises his left hand and he places it onto my right shoulder. It is only there for a few seconds before he takes it away. He then turns his body away from mine and focuses on the race that is underway. I am expecting him to say something, instead he says nothing. I wonder if that is it.

Is he finished with me?

I wait momentarily, just in case it isn't. But then I realize that his interaction with me is over. I feel angry, cheated. I cannot believe he has nothing to say to me after the biggest disappointment of my life. I sit on my own in the grandstand with my club mates around me. Nobody says anything to me, I feel incredibly lonely.

\section{Carly}

"14 years old"

\section{Part 2}

I sit in the lounge room at home watching the closing ceremony of the Olympics. My body is still filled with sorrow and regret, having missed out and not been a part of it all. It has been so hard watching the Olympics and knowing that I was so close to making it. Trials were 12 weeks ago now, yet my questions still remain unanswered. Tom never spoke a word to me after my race. Even on the plane on the way home, nothing was ever said. He then went away as he was selected as one of the coaches on the Australian Olympic team so I haven't seen him. Ifeel cheated. He is like a father to me. I invested so much faith and belief into his methods and what he told me to do. I want to know where it went wrong. Why was I four seconds outside of my personal best time when I was supposedly in the best form of my life? I suppose I will find out soon because he will be back at training next week. 
Before I know it, Wednesday has rolled around. Although I still remain bitterly disappointed over my performance 12 weeks ago, I know I will finally get some answers today because Tom is back. Mum drives me to the pool. I get out of the car and walk into the facility. To my surprise, I see Clay, the assistant coach standing there and Tom is nowhere to be seen. I wonder if he is in the toilet. I walk towards Clay.

\section{Carly: "where is Tom?"}

Clay: "He has decided that he is going to retire. He doesn't want to coach anymore. I am going to be coaching you now."

Carly: "what? So, that is it? No good bye, nothing. Is he done with coaching?"

Clay does not answer me. I am fuming. I have not got the answers that I wanted. I deserve to know what happened. I gave up the last seven years for that race. Seven years of spending seven hours a day slogging up and down the pool with Tom watching on. We paid him coaching fees and I get no good bye and no explanation for his sudden decision to retire. My questions remain unanswered.

\section{Jenny}

"16 years old"

The first event that you compete in at any meet is always the hardest mentally and today is no different. The World Championships is my third time to represent Australia and one would think that competing at such a high level would have become second nature to me, but it hasn't. Actually, I think it is getting harder. Before, competing for my country felt like swimming at any other carnival. Now I realize the prestige and honour associated with representative teams and with that comes a whole set of new tensions and pressures. Faces have become more familiar as well. What used to be a face that was unrecognizable is now a face that is familiar to me. I am noticing the Olympic gold medallists and world record holders that surround me. This intimidates me.

My three team members and I gather in the marshalling area ready for the heat of the $4 \times 100$ metre freestyle relay. There are a lot of nerves associated with this 
race and as a consequence, the four of us sit quietly. Our fastest 100 metre swimmer is being rested this morning. She will be inserted into the relay team tonight for the final. As a consequence, one of us will be dropped from the team. This will be decided from this morning's heats. Out of the four of us, whoever swims the slowest this morning will not be included in the team for tonight's final.

I am the first swimmer - leading our team off. There is no hiding when you are the lead swimmer as the audience and coaches know exactly where you are positioned. The four of us line up at the door of the marshalling area and then we follow the other teams onto the concourse. We stop behind lane three. This is the lane that we will be competing in. The other three swimmers swing their arms while I take my tracksuit off. I look over to my competitors standing beside me in the other seven lanes ready to start. They are tanned, toned and fatless. My body stiffens with anxiety. I know what this means. They are going to swim fast. I then look back at my team mates and instead of having a feeling of camaraderie, I see them as my competition as one of them could take my place in the final tonight if I am not careful.

I stand behind lane three. The referee blows the whistle in preparation for the start and I stand onto the blocks. The starter gets the eight of us away cleanly. I feel strong in the water and power down the first lap. Four girls are just in front of me. I turn fifth at the 50 metre mark. As I am swimming up the pool in the second 50, my arms start to fatigue. They feel so heavy. I am exhausted.

Think I went too hard in the first 50 metres.

I try and push through it, knowing that if I slow down, it could cost me my position in the final tonight.

Don't want to be dropped.

I have worked hard for this spot.

Don't want my team mates to beat me.

I put my head down and power to the wall without taking a breath. I touch the wall and the second swimmer in the team dives into the water. I look up to the scoreboard and see my time. 
58.05 .

Gosh, it felt faster than that.

Panic overcomes me. I don't think that is going to be good enough to make the team for the final tonight. I suck in the air, trying to get my breath back. It seems that I can't suck it in fast enough. I duck under the lane ropes to exit the pool from the side. I pull myself out of the pool and as I walk back to wear my gear is, my arms and legs shake with exhaustion. As I reach my towel, the second swimmer from my team touches the wall and the third swimmer dives into the water. I look at the scoreboard for the split.

\subsection{4 .96}

I try and do the calculations quickly in my head wondering what split my team mate has just done.

Gosh, she swam around 56 seconds.

That is faster than me.

I stand there, stiff with anxiety, waiting for my third team mate to touch the wall, almost willing her to slow down. She touches the wall the fourth swimmer dives in. I look at the scoreboard to find the split.

\subsection{0 .07}

Hell, another 56 split. I am going to be dropped.

I hold my breath, hoping and wishing that our final team member has a shocker and slows down. Instead she makes up a place, touching the wall in fourth place. I look at the scoreboard to read our overall time.

\subsubsection{7}

The final swimmer has split around 55 seconds. I know what this means. I walk back with my team mates to the warm down pool. A coach and team manager is waiting for us. We all gather around the coach who has our splits written on the program. It is now clear that I am the slowest. Nobody says anything to me. They don't even officially tell me that I am going to miss out on the final tonight. They 
just assume that I would know, which I do. I dive into the warm down pool and cry.

Am such a failure.

After the warm down, I get out of the pool and get changed. I head back up to the grandstand where the rest of my team mates are sitting. When I arrive to where Australia is sitting, nobody says anything to me, not the coaches, not the managers and not even my team mates. I sit there alone.

\section{Jenny \\ "17 years old"}

I touch the wall. The scoreboard reads 1.05.15. The time is slightly outside my best, 0.10 of a second to be precise but I am not worried because backstroke is not my specialty and my main event is not for another three days yet. I am lucky enough to make lane 8 in the final. I scan the crowd to find my parents. I see them. My Dad's face is expressionless and my Mum's face has a disappointed expression. The muscles in my stomach clench until it feels like there is a large knot. This feeling has become all too familiar around competition time.

A sense of dread overcomes me as I climb out of the pool. I take my time to collect my gear and warm down in the training pool. Other coaches and swimmers come over to congratulate me on my swim but what I thought was initially a good swim has been tainted by the disappointment in my parents' expression. I know all too well what her facial expression means. I complete my warm down and take as much time as I can.

I approach my parents with trepidation. I sit down beside my father. Without saying a word, he stands up and sits down on the other side of me so I am now sitting between my mother and father. I sense her disappointment. Her lips then move towards me and she whispers in my ear.

Mum: "What happened? That swim was a bit disappointing."

Her voice lacks warmth.

Jenny: "what do you mean?" 
Mum: "You also look like you have put on weight."

I try to ignore her comments. I know she is disappointed in me. I focus my thoughts back to the job that I have come here to do and that is to make the Australian Olympic team.

\section{Jenny}

"18 years old"

My muscles tingle as I walk inside the centre. The familiar stench of chlorine greets me. It has been eight months since I have competed - eight months since the biggest failure of my career, where I failed to make the Australian Olympic team. I was planning on giving up swimming, particularly competition but after a few months out of the water, I decided to try my hand at something completely different and give triathlons a go. The training that I have been doing for the triathlon has really helped my swimming. So, I thought it would be fun to enter state titles, catch up with some old friends and have a bit of fun. I am excited about seeing my friends again, friends who have been a part of national teams with me. I am also excited about catching up with a few of the coaches.

I walk into where the warm up is. I see a lot of familiar faces. I smile at a coach, one that I have been away with on Australian teams. He is a coach that I worked with on the 1991 World Championship team as my own coach had been placed with the long distance swimmers. We worked well together. As our eyes meet, he turns away without so much of a smile or acknowledgement.

Did he purposely turn away or didn't he see me?

I sit down and stretch. I put my earphones in my ears and listen to music because I feel uncomfortable. I cannot understand why I have this feeling of uncomfortableness as this environment was one I once strived to be in, one that I loved being in. A group of four female swimmers from another club approach me. Three of them I have been on Australian teams with. I smile at them, an invitation, or so I thought for them to come over. They giggle and whisper to each other and keep walking. This dishevels me, I feel like I don't belong. It is like I am no longer 
accepted, that I have become an outsider. I question what I am doing here and contemplate leaving.

I try to ignore the smirks, the whispers and the snubbing and remind myself that I have returned to racing because I am curious to see how I fast I can swim without the pressure of others' expectations. I am in the first event so as soon as I complete the warm up, I head straight to the marshalling area. As I am being marshalled, I notice that some of my male triathlon friends are in the grandstand; they have come here to support me. This brings a smile to my face. I sit in a row of seats next to my competitors who I am racing. One of the girls who I have roomed with on previous Australian teams, a person who I considered to be a friend notices me waving at my friends in the grandstand. She looks at me and says:

Paula: "Are they your boyfriends?"

I do not respond to her question. Her comment dishevels me and a state of melancholy overcomes me. It is not so much the words of her comment, it is more the fact that she failed to acknowledge me before making the comment even though she walked past me in warm up. Up until today, I had considered her to be a good friend - somebody that I could confide in, someone that I thought that I had camaraderie with. As fellow team members of numerous Australian swimming teams we had shared in something unique and now it seemed as though nothing had ever been shared.

We line up behind the blocks for the start of the race. Ifeel a little nervous but more excited about racing - a contrast to how I used to feel. The gun goes off and I dive into the water, everything that I need to do comes back to me automatically almost as if I had never taken the time off from racing. I feel good as I swim. I finish the four laps strong. I touch the wall and look up at the scoreboard. I have finished second in a time that is only one second outside my best. Ifeel excited, happy about what I have just achieved. My training load has cut down from 90 kilometres a week to about 20 kilometres, along with a few runs and rides, yet I still have managed to swim a fast time. I look up into the grandstand to find my friends. They are jumping with joy, so excited by my achievement. 


\section{Mandy}

"15 years old"

I look up into the steep stand of the Valley pool and I see my Mum and Dad sitting there amongst the crowd. I smile as my eyes lock with Dad's. He is sitting beside Mum and has come today especially because I have asked him to. I have something special in store, am feeling good and know I am swimming fast and want both Mum and Dad here to share it with me. Even though it is National titles, a high pressure meet, I feel confident.

I stand behind the start blocks with the other seven finalists for this event. I shake my arm muscles, loosening them off, trying to keep the tension associated with my favourite event at bay.

Mustn't be too confident, there are still people that are trying to knock me off my perch.

This is my event and I don't want anyone to take it away from me. Only last night, I won silver in the 100 metres.

It should have been gold.

I put last night out of my mind and focus on the race ahead. I love racing the 200 metres and I am going to show everyone what I am made of.

The whistle blows and I dive into the water in preparation for the start. I crouch up into the start position. My muscles are tense, alert in anticipation for the sound of the gun. The gun sounds, I take off. I control myself, careful not to expend all of my energy in the first lap. I swim strong, building each 50 metres as I go. I come in for the final lap noticing that I am well ahead, in fact over four metres in front of the rest of the field. I touch the wall. I know it is fast as I have clearly beaten the rest of the field. I look at the time keepers trying to hear what time I have done. By then, the rest of the field has touched the wall. The whistle sounds soon after the last competitor has finished. This is the signal to exit the pool. Soon, after I exit, I find out my time. I am so pleased as I have done my best time by almost two seconds. The only down side is that I have just missed the Commonwealth record by a tenth of a second. My time ranks me at number one in 
the world. Even though it is my best time, when reflecting, I realise there are certain areas where I could have improved, areas that I will work on for the Commonwealth Games in a couple of months. I am sure that at the Commonwealth Games when I am racing head to head with some of the other competitors from other countries, that I will be able to dig that extra bit deeper in the final lap.

I dry myself off with the towel and pull my tracksuit on to keep warm. I grab my belongings and then climb up the stairs of the grandstand to see my coach and family. I am keen to celebrate and share my victory with them. Am really pleased with the swim and what I have achieved - did a best time, won a national title, broke the national 100 metre backstroke record during that 200 metre event. So, all in all-not bad. As I approach my coach, I can see that he is not happy.

Surely he is not upset with me?

I approach him first.

Bob: "what was that?"

His voice is raised. It is loud enough that a lot of people in the surrounding seats can hear what he is saying.

Mandy: "what do you mean? It was a great swim."

Bob: "you swam like an amateur then. There were just so many mistakes.

That was terrible. You are hopeless."

Mandy: "I realize there were a few areas where I could have improved but I did swim my best time and I did just win a national title."

Bob: 'It was an amateur performance."

My heart sinks. I look around and notice that a lot of the people have just heard him tear my swim and me apart.

Feel embarrassed, ashamed of myself.

I get angry. Feel like punching something, someone but I refrain. 
I head over to my Mum and Dad. They give me a slight smile. They have just heard what has gone on. I sit down in-between them both. My Mum pats me on my leg but does not say anything. They can see that I am visibly upset from what my coach has just said to me.

I was on top of the world less than five minutes ago. Now I feel like my confidence has hit rock bottom. I put my face into my hands as my eyes are brimming with tears. I will not let anyone see me cry. I wait until the car and the tears start to cascade down my cheeks.

\section{Mandy}

"14 years old"

I touch the wall, completing the warm-up and find a position in the lane where I can tread water without kicking my team mates. Everyone is waiting, wondering what the main set will be today. I look at my coach, trying to find some clue to what we might be doing. Then he starts to speak.

Bob: "You have 4 x 1 miles descend. Form stroke ${ }^{19}$ swimmers are to do it form. I want it descend though. Each one has to be faster than the one before. Let's get going!"

Each swimmer in the lane works out their position according to their speed and the stroke they are swimming. I am doing form so I am fifth in the lane. I push off and settle into a rhythm quickly for the 32 laps ahead. I feel strong as I work my way through the laps. I touch the wall on the $32^{\text {nd }}$ lap of the first mile and lift my head to hear my time. It is quick, only a few seconds outside my best. I get 30 seconds break before I push off again. Again I settle into a rhythm quite quickly. I feel strong but I am quickly becoming tired. I know I am swimming quite well but I think I have gone a bit too hard. Towards the second half of the second mile, my body fatigues heavily. I feel spent. I have gone too hard and I am aching all over. I feel like my body is breaking down. Don't think I can go any further.

\footnotetext{
${ }^{19}$ Form stroke swimmers are swimmers who have a focus stroke other than freestyle. For example, backstroke, breastroke, butterfly or medley [combination of all four strokes].
} 
I touch the wall on the $32^{\text {nd }}$ lap and again lift my head to hear my time. It is fast, my best time. I break down with tears from the exhaustion. My coach sees me. I look at him directly.

Mandy: "I can't go any further."

Bob: "If you don't keep reducing your times, we will keep going until you do."

My shoulders drop under the water. Instead of feeling inspired, I feel deflated, demoralized. I feel like getting out of the water but I know that my coach has produced champions in the past and I have to do what he says if I want to be the best. I push off the wall for my third mile and my body is aching, really hurting. I cry as I swim and I try desperately to suck the oxygen in because I am puffing so much from the exertion. I finish off the final two miles, descending them as my coach stated, avoiding repeating the entire set. I am still crying. Without warning, my body starts to convulse and I vomit up the content of my stomach. He sees me but doesn't say anything to me, nothing about my exertion, about me achieving his expectations. At the end of the session, I climb out of the pool. I don't feel a sense of achievement, but instead feel beaten, affected by my coach's words. 


\section{ACT 3 \\ TALKING ADULT BODIES: EFFECTS OF A PERFORMANCE AND PERFECTION FOCUSED CULTURE?}




\section{ACT THREE - Sequel}

Prologue: Carly is married and is a mother to a three year old girl and four year old boy. I have since married and have two boys aged seven and four. Mandy is recently divorced and has no children. Mandy's story of experience is written in her final year of marriage.

\section{Carly}

"2009"

There are five left. I wish he would go to work. Need to have them.

Carly: "When are you leaving?"

Casey: "In a couple of minutes."

I help him to get his things together and kiss him goodbye. I wonder if he noticed me rushing him off to work. I walk back inside the house. I check on the kids. They are watching television. This is my opportunity as nobody is watching. I open the pantry door and I stare at them. They are in the glass container on the shelf. I have a battle.

Should I?

\section{Shouldn't I?}

I know that I shouldn't. I know that they are unnecessary calories. I know that they will make me fat, yet I wonder why I am having trouble resisting.

Have to sneak them without the kids seeing me.

That is so stupid.

I rationalize my thoughts.

Why would my babies care if I eat a biscuit? 
In fact, why would Casey even care?

The inner battle continues momentarily.

Don't eat them!

I really want them!

The inner battle is over and I succumb to temptation. But, instead of taking just one biscuit, I grab the five of them out of the jar. I walk into the lounge room so the kids don't see me and I shove them into my mouth one by one, until the last one is eaten. The sweet sugary taste gives me a nice feeling, but it is only momentarily. Then my voices start.

You are fat!

You are disgusting!

Some familiar words come back to me, words that I have not heard for a long time but resurrect occasionally.

Winners aren't weak.

Winners aren't fat.

I am so weak. Feel really terrible now for eating the biscuits.

(McMahon et al., in press)

\section{Carly}

“2009”

I walk into the bathroom and I see them. I have placed them in the middle of the bathroom floor as a reminder to me. They haunt me every time that I walk in there. Even though they haunt me, I have purposely placed them there to remind me not to put on weight. Every time that I feel like eating chocolate, I go and stand on them, weighing myself as a reminder to what will happen. As I stand there looking at them - ready to stand on them, one of my voices starts. 
I ignore my voice and step onto them for the second time that day. Before looking down at what I weigh, I stare ahead out of the bathroom window, trying to rationalise my behaviour.

Carly, you would not have put on weight in two hours.

Don't do it to yourself.

Even though I realize that my constant weighing is a method of self-torture, I cannot stop.

I look down at my weight. I am 0.25 of a kilogram heavier than what I was two hours ago.

Heaviness drapes over my shoulders. Ifeel deflated.

I hate myself.

I am disgusting.

I walk outside; my kids are still watching television.

Maybe I did not read my weight correctly.

So, I walk back into the bathroom to check again. This time, unbeknown to me, my daughter has followed me. I steadily step onto the scales cautious that any slight movement could cause an increase in my weight on the scales. I look down and my weight is the same.

They are not wrong.

I get off and as I do, Kylie pushes past me and steps onto them, modelling my carefulness, my trepidation. I am horrified that she has seen me, that she is modelling me. I wonder what damage I have caused her.

Don't want her to go through what I have!

What have I created?

(McMahon et al., in press). 


\section{Carly}

"2009"

There are only a handful of people that I can rely on in this world. These people are so important to me and I love them more than life itself. Once I allow them inside my circle - they become my life - my everything. My head is spinning today. I am upset. Someone within my circle has let me down.

I wonder if I should say something. I want to, but I am scared-even fearful.

It just seems that I have been putting in all of this work and I am not doing anything right.I mustn't say anything. I don't want to lose them. I do not want upset them.

Am terrified that they will leave me.

I decide that I should not say anything. As the hours of the day pass by, I sit at home thinking about the situation and as I do, turmoil builds inside me. I have an internal battle about whether I should express what is upsetting me.

Everybody I disappoint in my life ends up upset or leaving me.

Mustn't upset or disappoint.

My mind drifts back to when I was 14 and I upset my coach. It was the time when I should have made that Olympic team and didn't. He put so much work into me and when I failed him, he ended up leaving me. That experience at Olympic trials reminds me that I should not say anything.

If I disappoint, they will leave me.

I have a battle with a voice in my head.

You are being stupid. You need to say something. You can't hold it in forever.

The next day I run into them. As they are standing in front of me, the words spill out of my mouth, without intention - almost automatically. I say my piece - what I 
am unhappy with. It does not go down very well. As I see their look of disappointment, even anger, I become panicked.

I have done the wrong thing.

What happens if they leave me now?

I have got it all wrong

I am being a selfish bitch.

I am too critical of them.

Have made a big mess of things and just have to make it right again.

Am so ashamed of myself and have totally disrespected them.

What have I done?

\section{Carly}

“2005”

Will I finally get my answers today - more than a decade on?

Another school year has passed and it is the awards ceremony tonight at school. The school has organised a special guest to come and present all of the awards to the students. It is Tom, my coach, the man that I considered to be my second father as I was growing up. He is the man that I have not seen since that fateful day of Olympic trials. It was the day when my lifelong dream ended. It has been over a decade since I missed out on competing at the Olympics. It still haunts me. The hardest part is trying to understand why? My questions still remain unanswered. We have never spoken after that day, he never contacted me to answer my questions or even to say good bye. I start to feel butterflies in my stomach - an excitement of sorts, in anticipation of seeing him finally and talking about that day when my dream ended.

The night is finally here. I walk inside the school hall - the facility where tonight's function will be held. My eyes scan the room - in a purposive fashion - trying to find Tom. 
I see him. He is standing with the principal of the school and they are chatting. I walk towards him and as I do, my heart races. I am not sure why I have suddenly become so nervous. Our eyes connect. He smiles and greets me. Our conversation has no depth but rather, contains polite formalities. Tom is friendly to me but there is no connection - not like there used to be. I stop talking and look at him. Although I never ask him directly for answers, I wait - pausing in anticipation for the answers that I have been waiting for. Instead he says nothing. I wait longer but still nothing.

Maybe he is trying to find the right words to say to me?

He notices that I am staring at him. His eyes connect with mine but then he looks away.

Tom: “Better start mingling, it will be impolite if I don't go and say hello to everyone."

He walks off. I get no answers.

Why didn't he ever say good bye to me on that day so many years ago?

I trusted him as a coach - as a person. I loved him like a Father.

Why did he leave me?

Why couldn't he manage to find some words to say to me after the biggest disappointment of my life?

Why didn't he give me answers today? I have been patient.

His silence that day when I was 14 saddened me and his lack of acknowledgement tonight does the same. I felt so isolated and abandoned when I was 14. Tonight is no different. Tears start to fill my eyes. I rush to the bathroom before anyone can see me.

Don't I deserve more than just a pat on the shoulder?

Pain and hurt fills my body. It hurts just as much as did when I was 14. 


\section{Carly}

“1999”

It feels like I can call London home now. Have already been teaching over here for nearly a quarter of my 12 month contract and I am loving the lifestyle of the big city. The school has even offered to sponsor me to stay in London which I want to take up. I like teaching over here but I am sick of not being paid what I should be. I was teaching for five years in Australia, yet over here, I am being paid a first year teachers' wage. Subsequently, I feel like I am also being treated like a first year teacher. This morning, I took the payment issue up with the principal of the school where I am working. She told me that to get paid what I deserve, according to my experience, I need to complete an overseas teachers' training course. The thought of have having to complete a course panics me.

There is no way that they are going to make me do it.

Why should I have to prove myself to them?

What happens if I fail?

After this morning's meeting, I felt confused, which shortly turned to anger. Nobody told me that when I came to London that I would have to sit an exam. I completed a degree successfully in Australia. Why do I have to prove myself again? My anger starts to turn to self-doubt.

What happens if I decide to do it and don't pass?

I don't want to do this, but I have no other option if I want to get paid more. For the days that follow, I become anxious every time that I think about it. I contemplate working in a bar instead as I am so scared of the prospect of failing. Even though I haven't seen my swimming coach for ten years, his words still clearly ring in my ears.

Failure is not an option

Failure is for losers. 
Am so worried about failing this exam. Failed in swimming, don't want to fail this.

\section{Jenny}

“2009”

I am frustrated. We have had another tiff. I hate fighting with Chris, my best friend, my husband. It seems that we have been fighting about this more and more lately. Chris found some McDonald's wrappers in the car. They were 'McHappy' meal boxes that could not be mistaken for mine but were a lunchtime choice that I had chosen for the boys one day as a treat.

Chris: "Why do you feed the boys crap food when we have the opportunity and money to feed them healthy options?"

I become angry with him. I feel like he is surveilling me, monitoring the choices that I make for the kids.

Jenny: "So, what! I gave them McDonalds. It is not that bad; it is not as though I give it to them every day. Stop monitoring me. I am not going to deny them things that I was denied! You need to get over it!"

Chris: "Jenny, what about the chocolates that you bought them yesterday? It would be ok if it was just once a week but you treat them every day. Your treats are becoming their diet."

Jenny: "You cannot control me! I am going to treat the kids if I want to. I don't want them to miss out. You can't stop me. Stop trying to control me. Stop gazing; stop monitoring my parenting and me."

Chris: "I am their father Jenny. I have a say in what goes into their mouths. You are feeding them too much crap. Please stop."

Jenny: "No, I am not going to stop. You can't make me."

I walk off in frustration, angry at what has just happened. I feel so strongly about my beliefs and so does Chris. I am not willing to compromise and nor is he. We lock horns for the second time this week. 
(McMahon et al., in press).

\section{Jenny}

“2007”

I open the heavy glass door. A waft of deodorant and old sweat hits me. A young and muscularly defined male gym instructor is standing behind the front counter. Our eyes connect. He flashes his pearly white teeth in a huge smile. I can feel his eagerness. His body appears rigid, in anticipation of how he can be of service to me.

Jenny: "Hi, I was just wondering how much your membership prices are?"

Gym instructor: "Have you done gym before?"

Jenny: "Yes, but not for a while."

Gym instructor: "Are you interested in a three, six or 12-month membership?"

Jenny: "I think I would prefer to start off with the three month."

Gym instructor: "The three-month pass is \$299; it includes full use of the weights, all classes and an individualised program. Here is a copy of our class timetable. One of our instructors will meet with you and talk about your goals, take your weight, measurements and work out your percentage body fat so we can track your progress over the three months."

Without warning, a lump appears in my throat, a feeling of panic overcomes me and my heart rate increases. I can feel pounding in my chest. It is like I have dropped the reins of the horse that I am riding and my control has been ripped away. My eyes fill with tears. I feel embarrassed and self-conscious of my emotions.

What is wrong with me?

He is going to think that you are a psycho Jen.

I try to hide my emotion by speaking as if everything is fine. I giggle. 
Jenny: "That sounds great; I will think about it and get back to you."

Got to get out of here!

I get into my car and drive to the university in a state of melancholy. I feel disconcerted, dishevelled emotionally by what would seem a normal interaction. I walk into university and quietly sit down, a contrast to my usual self. I make no eye contact. I don't want anybody to come and talk to me. Am scared I will totally lose it. Anger takes over.

Just breathe Jen, hold it together.

What the hell is wrong with me?

Why would that guy presume that progress and success is measured through the downsizing of my body?

What would he know?

No one is ever going to take my weight, measurements or calculate my percentage body fat!

He can get stuffed! They all can get stuffed!

(McMahon, 2007)

\section{Jenny}

“2008”

The nurse is engaged in a flurry of activity around me, checking that my patient arm band matches my file and making sure that I have signed all of the necessary forms. She asks me one question after another, often repeating questions to make sure she has recorded the correct information. I cannot believe the paperwork that needs to be filled out for just day surgery. She looks like she is finishing up when she turns to me and starts to speak.

Nurse: "Ok Jenny, the anaesthetist will be here to see you in a moment. I just need to get your weight before he gets here." 
She walks off before I can respond. Panic immediately surrounds me. My heart rate increases and the room starts to spin. It is stressful enough that I am in hospital and am about to have day surgery, but this is something that I was not expecting. I have not weighed myself in over nine years. I purposely have avoided the scales. They are an instrument that brings me so much turmoil; so much heart ache and subsequently, I do my utmost to avoid them. I know they are just an object but I fear them and most of all I fear myself around them.

My stomach starts to get heavy as I start to feel like I am going to be sick. My head starts to pound with the pressure. The nurse arrives back carrying some scales.

Jenny: "why do I have to get weighed?"

Nurse: "the anaesthetist needs to know your precise weight so he can administer the correct amount of anaesthetic."

Jenny: "I don't weigh myself and I don't want to weigh myself. Is there some other way?"

Nurse: "I need to get your weight but I don't need to tell you your weight." I ponder her suggestion before agreeing. I carefully stand on the scales, looking straight ahead, without so much of a glance down.

Nurse: "ok, got it."

She writes it into my notes, but I quickly look away just in case I can make out the shape of the number she is writing down.

\section{Jenny}

“2009”

\section{Part 1}

Another operation, it is the third in 14 weeks. It is only ten days away now. The procedure is consuming me, permeating my thoughts and my being. This one is the big one and the other two that I have already had in the last few weeks seem 
minor in comparison. My oncologist will be performing a hysterectomy on me, at 34 years of age. I have struggled to come to terms with this one. Not because I am having something removed, but, because of the act of the hysterectomy, the operation, the act of surgery being performed on my body.

\section{I am terrified.}

I have become very protective of my body and this procedure is totally out of my comfort zone. I have expressed my feelings and my fears to my doctor. She is so understanding and has listened to everything I had to say. She has given me two choices for the surgery but they have contrasting recovery times and are dissimilar in method. The first choice, a laparoscopy is less invasive and apparently has a quicker recovery. The doctor will make three incisions into my stomach and will fill my belly with carbon dioxide gas and they remove my organ with minimal impact. However, through this process, I am asleep and I am paralysed, intentionally done so to assist the procedure. A machine will be breathing for me; my body will no longer be able to function on its own without the help of machines. I will be asleep and I have no option of being awake during this operation. I will have no voice, no choice, and no power. I cry as I write this, my body starts to shake with fear. I wonder why I am so fearful of this. I have tried to discuss it with others and they tell me not to be silly, that they would choose to be asleep, but for me, I am uncomfortable with surrendering my power, my bodily functions, my life to others, having no voice and no power through the entire procedure.

The second choice, I am more comfortable with as it can be performed with an epidural. I will be awake and alert and my body - my breathing in particular can function without any assistance. The doctor advised me that this would be a longer recovery and far more painful. She would make a 20-centimetre incision in my lower belly through my abdominal cavity and remove my uterus through the opening. This appeals to me, not the pain associated with this procedure or the fact that a large incision is made through my abdominal wall, but the fact that I can be awake and breathe on my own. I feel comfortable that I can still have some power through the procedure, rather than being docile, a body, a cavity functioning without my-self reliant on the assistance of machines. 


\section{Jenny}

“2009”

\section{Part 2}

I sit on the operating table in the crouch position. A young guy is holding me still as well as holding my hand at the same time. I feel a sharp pressure like feeling in my spine as the local anaesthetic is administered. It is only like a matter of a minute after the first needle before I see a huge bore needle out of the corner of my eye, approaching my spine. I feel an uncomfortable pressure, I want it to stop. I hold myself still, terrified that the anaesthetist will puncture my spine with one slight movement. Having told me all of the risks, they run through my mind frantically as he pushes the big needle into my spine. He keeps pushing it in further and further, this worries me. After a couple of seconds, I feel relief, not because the needle is in place but because he has pulled it out.

Anaesthetist: "I am going to have to do that again. I did not have a good position. Try really crouching over."

I squeeze the young guy's hand that is holding me really tight. He squeezes extra tight and as he does, I know what this means - the big needle is close. Ifeel the pressure again. I try not to move, staying really crouched over. It is over quite quickly.

Anaesthetist: "Ok Jen, swing around and lift your legs up."

I am surprised as my legs have already become paralysed and I can't really seem to do what the anaesthetist has asked of me. The young guy helps me to lift my legs up. I lie there, scared about the procedure ahead. The surgeon walks over to me. She places one hand on my body and starts to speak.

Surgeon: "are you feeling ok Jen?"

I nod because I am too nervous to speak.

They put a barrier up so I cannot see what is happening. It is really close to my face and I feel claustrophobic. It is not a nice feeling and I become somewhat panicked. 
The anaesthetist gets the surgeon's attention to let her know that she can start. I feel the first cut. It is not sharp but it feels like I am an orange with multiple layers being prised through. I can feel the layers being exposed, a sizzling as each layer is being cauterized. It is just too much. She is only minutes into the operation before I ask her to stop.

Jenny: "this is really hurting."

Anaesthetist: "I told you Jen that you will feel some pulling and poking."

Jen: "No, it is more than that. I have had a caesarean before and this is hurting more than just pulling and poking."

Surgeon: "what can you feel?"

Jen: "I feel like I am an orange and that you are cutting a whole series of layers and as you cut through each one, there is a slight pop. I can also feel sizzling as if you are burning something. "

Surgeon: "do you want a sedative."

Jen: "Yes."

The anaesthetist injects a cloudy substance into the cannular in my arm. The doctor waits a minute or so and then recommences. The sedative makes no difference. It is just as uncomfortable. I look at the clock, trying to distract myself. I want to go to sleep but if I do, my body will have no power. I have an internal battle.

Don't want to go to sleep.

Don't want to give my power away.

Need to be able to see and hear what is going on.

My body needs to have a voice.

Have strength Jen, you can do this.

A minute has not even passed before it becomes too much. 
Jen: "STOP."

Surgeon: "I am not going to do anything Jen without your permission."

Jen: "it just hurts too much."

I call the anaesthetist who is sitting behind me. I want to talk to him but I need to see his face.

Jen: "I want to go to sleep but I don't want to be paralysed."

Anaesthetist: "Jen, this is not an ideal situation. I will be giving you some heavy sedatives and it is just too much to ask your body to breathe on its own. It is going to be working against the sedatives. It is safer to be on the machine."

Jen: "I do not want to be on the machine. I can do this."

Anaesthetist: "Ok, I am happy to give it a try but the moment you are compromised, I am putting you on the machine."

I nod and as I do I am waking up as it is all over. My immediate attention is placed on my stomach. It hurts.

Anaesthetist: "You did really well; I did not need to put you on the machine to breathe. You breathed on your own the whole way through."

\section{Jenny}

“2008”

My embodied experiences, in particular my swimming experiences constantly haunt and taunt my body, in particular my relationship with food. Even when I am teaching, I find myself battling with my inner most demons.

As I walk towards my first class for the semester-Body, Identity and Culture in Sport, I see a bunch of students inside the room with unfamiliar faces. I feel nervous even though the content of this subject covers many of the issues that I faced as an adolescent swimmer and later in post-career as a woman. My experiences are so relevant to this subject therefore I am going to try my hardest 
to incorporate them into the content by linking them to the theoretical concepts, the practices and the pedagogy included in the content. I want to share conversations and experiences that I have personally encountered during my swimming career and the affect that they have had on me with the intention of enlightening the students.

As I open the door to tutorial room, 30 eyes suddenly focus on me. I am sure that it is not my paranoia, but I notice a few of them scanning my body. They all look so fit and sporty. I suddenly feel self conscious. I used to be an athlete, but my body no longer looks like it used to. It is does not look like it even fits into their discourse (Human Movement and Health and Physical Education educators). Even though I have had the sporting experiences and have acquired sporting knowledge that I am able to share, Ifeel as though my body does not measure up to their standards - that it does not fit and that I should not be here. I subsequently feel like an outsider, out of place in this setting and this makes me feel uncomfortable before I even have spoken any words to them.

After the introductions at the beginning of the tutorial, we move onto the first activity. I ask the students to draw and label an image of what they believe constitutes a healthy body. My intention for this introductory activity was to gain a better understanding of the students' philosophy in regard to health which in turn would affect their own pedagogy as future health and physical education teachers. I expect the students to draw a body, labelling it to not only include a healthy body (free of sickness) but to include healthy emotions, spirituality, thoughts, sensations, experiences and conversations.

After ten minutes of them drawing and labelling their drawn bodies, I initiate a group discussion and ask for volunteer students to list their beliefs of what constitutes a healthy body onto the whiteboard. The following list is written on the board;

- Not fat

- Muscular

- Doesn't drink much beer

- Doesn't smoke

- Does weights 
- Exercises a lot

This list immediately angers me. As the anger pervades my body, I try to breathe and not show the students my emotions. I battle with refraining from saying something to them. I am dumbfounded that as future health and physical education teachers, the very people who will have a large influence on adolescent bodies in a sporting and health related context could have such a narrow focus in regard to what constitutes a healthy body.

Jenny: "What about wellbeing. Let's talk about that."

I am still so angry.

Need to get out of here. Can't just leave.

I ask the students to talk amongst themselves while I pretend that I need to get something from my office which is only 25 metres away.

Get it together Jen. This does not look good for you as a teacher, if you cannot keep it together.

I walk back to my office and get a drink of water.

Just breathe Jen.

I am only gone for a matter of minutes before I return. When I walk in through the door, I see that 'happy mind' as been added to the list. Without saying a word, I write the following passage on the whiteboard. Nobody says a word as I do this.

"A healthy body is about having a balance, being happy and healthy in everything that encompasses the body including, as body, emotions, spirituality, thoughts, sensations, experiences, conversations constituting the individuality and identity of each person, as a soul, underlying all subjective experience."

A student starts to speak.

Student: "But you said body, you did not ask us to talk about the mind."

I ponder my response momentarily. 
Jenny: "When I talk about the body, I view the body, the mind, your self and being as one - as one total, one essential, one entity that pertains to being a person, an individual."

I try to explain it simply - in a number of ways but they look at me blankly.

\section{Jenny}

“2008”

Up until six months ago, I had not exercised for seven years. Not so much of a throwing over of the arms in the water or even a walk up to the shops. I even avoided walking up stairs if I could. I recall my husband asking me if I wanted to go for a walk at the beach when we lived in Queensland. I could not be bothered to even leave the house, even though I could see that he wanted to do something with me. Ifelt tired, unmotivated and exercise was the furtherest thing from my mind. If I could avoid it, I did.

It was only after having cancer that I had an epiphany. I realised that my conscious choices of eating unhealthily and not exercising, was not actually me having the power like I thought. I was actually disempowering myself and my health.

It was after this epiphany, six months ago that I started exercising. I began with just walking, building up the amount of time that I spent exercising each time and the amount of times that I was doing it each week until I was eventually walking daily. For six months, I have built up my weekly training routine to the point where I now swim three training sessions per week (10 kilometres per week), run twice a week (16 kilometres per week) and walk on the days that I am not running. I love having my body moving again and continually set myself challenges. A few weeks ago, I completed the run leg of a triathlon; it was 12 kilometres in distance just as an act of challenging myself. It is like I have become addicted to exercise again. I refer to it as an addiction, because last week, I became frantic after missing a training session because my little boy was sick. Below is a diary extract of my thoughts after missing a training session. 
I go to the fridge to get a snack. I then remember that I have not trained today so I close the fridge. I am hungry but distract myself with daily chores. My husband finds me folding the washing and asks me what I want for dinner. I tell him that I have had a big lunch so I don't really feel like dinner. I lie to him so I can avoid having dinner. Mustn't have dinner, haven't burnt off the calories.

\section{Mandy}

"2008”

I am home alone now but Angus and I just had a fight. My shoulders are tight. My body feels tense. I feel low and worthless.

Need to try harder to please. Have to try and do more for him. I hate myself. Not sure how I can do more though because I am tired, in fact very tired. My body is telling me to stop, however I can't because I know it is a sign of weakness if I do.

I go over the argument in my head, wondering how I could have prevented it. It started because I was at a swim meet all weekend. It just happened that I was also 'needed' at my husband's company. As well as coaching, I also fulfil duties in regard to my husband's business. My typical day starts at 4.30am and does not end until after $7 \mathrm{pm}$.

Today, I was just so exhausted and decided to go home after morning coaching to have some breakfast and quiet time before the onslaught at my husband's business. When I walked in the door at 8.30am, I found my husband was also not at work. As soon as I entered, I immediately saw that he was seething. He was angry that I had not helped him over the weekend and that I was still was not at the factory yet. Ironically, he was not at the factory either. I could sense his anger through his body language. I knew what was about to come.

\section{Please God not again!}

Now that he has left and the argument is over, I feel sick. I feel incompetent, ungrateful and useless. I go to the fridge, open the door and look inside. I see 
bacon. I reach inside; take it out and start cooking it up. The pan is overflowing with the enormous amount that I have placed in it. I cook up some toast while I am waiting for the bacon to crisp up. Once it is ready, I pile it all on my plate and start eating. After I finish, I go to the freezer. As I open the door to the freezer. The freezing air hits my face as I look inside. A three litre tub of ice-cream is staring at me. I reach inside and grab it. I take the lid off and see that there is about three quarters of it left. I also spot the frozen chips. I have an inner battle momentarily.

Chips or ice-cream? Chips or ice-cream? Chips or ice-cream? Why not both? Yes, both are good!

I place the ice-cream on the kitchen bench to partially melt. Love it when it is like "Mr Whippy" soft serve style. I pull out a deep frying pan, grab the oil, heat it and start cooking.

Got to make sure the chips are cooked just right.

I go to the drawer and grab a fork for the chips and a spoon for my ice-cream. I pull the lid off the ice-cream and start to dig in.

This is not right. I need the chips first then the ice-cream.

I strain the chips from the deep fryer and then add lots and lots of salt.

Eat the lot, must eat the lot.

As I start to devour my way through them, I start to feel like I'm about to burst.

Please no, not yet, I have more to come.

Now, it's time for the ice-cream. As I swallow the first mouthful of ice-cream, I feel good, in control as I have felt throughout this whole process. The ice cream is so nice, so smooth and creamy. I keep digging in.

Ifeel like I'm about to explode.

I find myself yet again hanging over the toilet bowl purging (an old saying “driving the Royal Dalton”). As my fingers touch the back of my throat, my body 
reacts and I gag, then vomit. I do it again and again until I start to throw bile up, then I stop.

When I return from the toilet, I find that the tub's empty.

Must replace. Will do it at some stage today.

Have to make sure there is ice-cream for him. Everything can easily be replaced and nobody will know.

I go to the bathroom. I wash and scrub my face and then moisturise my skin. Need to scrub my teeth and gargle, removing all traces of my secret life. As I look into the mirror, heaviness drapes over my shoulders and that familiar feeling of selfloathing starts to take hold of my body.

(McMahon et al., in press)

\section{Mandy}

“2008”

I settle myself into the hospital bed. I look around and notice that the decor has not changed much in the 18 months since I was here last. There is a knock on my door and then a familiar face enters. It is the same nurse that looked after me last time I was here.

Nurse: "Hi Mandy. How have you been? I see you are here to have your other hand fixed. I just have to get some details from you and take your blood pressure. Can I get you to stand on the scales to start with? The anaesthetist needs to know your weight for your anaesthetic."

A familiar feeling of uncomfortableness overcomes me as I am asked to stand on the scales. I don't want to get on. The horrible dread always overcomes me every time that I have to share my weight with anyone. However, I end up doing as I have been asked. The nurse writes my weight down and then looks at me.

Nurse: "You go girl!" 
I wonder what she means by that comment. Does she mean that I have lost weight or put on weight? My mind starts racing as panic starts to overcome me.

I need to know how much I weigh. I want to know now. It becomes too much.

Mandy: "Can you tell me my weight please?"

She then puts the blood pressure cuff on and it seems like she has not heard me.

Mandy: "Tell me my weight please!"

Nurse: "Are you ok? Have you had a problem in the past with knowing your weight?"

I nod in response to her question.

Nurse: "You are 54.5 kilograms. Last time you were here we were really worried about you because you were 51.2 kilograms. You were underweight and the anaesthetist was worried what affect the anaesthetic would have on you. You are in the healthy weight range now."

I feel pleased. This reaction is a contrast to what I would have once felt as I strived to be underweight.

\section{Mandy}

“2009”

As I pack up the last of our things, I get the dog and we climb into the car. I have been looking forward to getting away for a while now. I am tired. Work has been particularly demanding lately and I am looking forward to sleeping in for the next few weeks. I play our favourite music and we are away. As we drive down the street, I find myself smiling about the holiday ahead. I lose myself in the music and my thoughts.

Carl: "Mandy!!!!"

Mandy: "yes" 
Carl: "didn't you hear me? I called your name four times."

Mandy: "I was a million miles away."

Carl: "No, I turned the music down and called your name and you still didn't hear me. Your hearing is getting worse. You should get it checked out."

I go quiet and so does Carl. I knew that it had been getting worse but I thought that it might be something that happens to everyone as you get older. But then I think about Carl and his hearing is fine.

My thoughts drift back to that plane trip, all those years ago when I was on my way to the Commonwealth Games.

Could it be?

Am I finally feeling the consequences of that plane trip from when I was a teenager?

\section{Mandy}

“2010”

I cannot believe how unseasonably cold it is. I walk into my wardrobe and look for my woollen trousers. They are black and stylish and have survived the fashions that have come and gone over the last 16 years. I pull them on. They don't seem to slide on as easily as I remember. I do up the zipper but it cannot be closed. I look down. I realize that there is no way that I am going to get the button done up. Then it dawns on me, like a sudden lightening strike from the sky.

I have put on weight!

My mind starts to race; I try and control my body's reaction through breathing. Those horrible feelings pervade my body.

I am fat!

I am ugly! 
I walk outside to where my partner is sitting. Tears are streaming down my face. I still try and control my breathing.

Carl: "Mandy, what is wrong?"

Mandy: "Look at my pants. They are too tight. I have put on weight!! What I am going to wear?"

Carl: "You are beautiful. Your body is looking healthier and healthier.

When I first met you, your hip bones were sticking out and you had a body like a teenage boy. Now you are curvy, feminine and just gorgeous. Can't you see that you are healthier?"

Mandy: "I suppose, but these pants have fitted me for 16 years."

I leave the room and go into my room. I battle with my internal demons. I push the self-hatred thoughts aside. I rationalize my weight gain with the fact that I am now a healthier person. This rationalization is a contrast to my previous behaviour of where I would react by purging. However, I realize for the first time in nearly two years that my body demons are still alive and well. They are just buried, deep down inside. I wonder when they will rear their ugly head again and whether I will ever be free from them. The thought of them returning scares me, but it is momentarily, because today I realize that I had a win.

\section{Mandy}

“2009”

I really hope the swimmers do well. I feel their tension. They have put in so much hard work. This is what we have been working towards all year. As I walk into the aquatic facility, I walk towards our usual meeting spot on pool deck, ready for the warm up. I am the first to arrive but it is only a matter of minutes before some of my swimmers start arriving.

As each of them walks towards me, they give me a slight smile. It is not their usual smile. They sit down and commence their stretching routine. They are not joking around as much as they usually do. After 15 minutes or so, they get ready to get into the pool and complete their warm up. I see them scanning the pool, there is 
so much action with everybody warming up, a flurry of coaches are rushing around on pool deck with stop watches hanging out of their pockets or from around their necks.

As my swimmers are readying themselves to jump into the water and start warming up, one of the male coaches makes eye contact with me. He has been a part of Australian representative teams before. We greet each other.

Mandy: "Hi, how are you going?

Male coach: "Yes, well. I am all ready for this week. How about you?"

Mandy: "It should be a good week. How are your swimmers going?"

Male coach: "Yes, good. I should have a few good performances here."

At that moment he glances over to lane six, where his swimmers are standing ready to hop in. He nods towards one of his stronger female swimmers and then starts to speak.

Male coach: "not sure how Missy is going to go, look at the fat arse that she has got now."

I cannot believe that he just said that. I wonder if I should respond. His comment infuriates me but instead of saying something, even after all of my own experiences, I say nothing. A sick feeling overcomes me and I just want him away from me.

Mandy: "anyway, I better get back to it. These guys are about to hop in."

I feel angry with myself. One of the reasons that I got into coaching was to ensure that the experiences that happened to me would not happen again, yet when a damaging comment like that was made to me, I said nothing. I just did not have the confidence to stand up to him. He is after all an Australian representative coach.

Once my swimmers complete their warm up routine, they go to the change rooms and change into their race swim wear. I head up to the grandstand to our usual spot and wait for the competition to start. One by one, my swimmers arrive 
dressed in their race swimmers with tracksuits on to keep their muscles warm. They sit around me.

Mandy: "Well, this is the first day of competition. You guys have worked really hard all season. Just relax and enjoy yourself when you are racing. You can only try your best, I am not asking for anymore. I am so proud of you all."

I look through the program. I notice that Katie is in the first event.

Mandy: "Katie, you better think about going down to the marshalling area in a couple of minutes, you are in the first event. Can you come and see me before you go down?"

Katie nods. I look down at the competition pool starting to empty out as the pool clears for the start of the competition. Katie gets up and grabs her bag and heads towards me.

Mandy: "Even though this is your main event, make sure you have fun swimming it. Try and get into a rhythm quickly. Make sure you work into and out of your turns. The eight laps will be over before you know it."

As Katie walks off, I start to feel nervous for her. She has worked so hard.

Hope she swims well.

One of the parents hands me a coffee; this distracts me momentarily from thinking about Katie and her race. Before long, the music on the public address system starts playing and the eight finalists for the women's open 400 metres freestyle final marches onto the concourse. The announcer introduces each of the finalists. Katie is in lane three as she qualified third fastest from her heat swim this morning. Once the introduction of the eight swimmers is over, the referee blows his whistle signalling the eight swimmers to stand onto the starting blocks in preparation for the race. They are now in the hands of the starter. The gun goes off and the girls dive into the water. Katie gets a nice start and gets into a rhythm quickly. After 100 metres, I notice that she is a bit tight in her technique and her stroke does not seem to be smooth. She looks like she is having trouble maintaining rhythm. At the 200 metre mark, she has dropped off the pace from the 
leaders and is coming fourth. Her split is three seconds slower than her heat split this morning. My heart starts to sink, only because I can see that she is struggling and not feeling good. She finishes the race in a time that is five seconds slower than her heat swim. She is also eight seconds outside of her best time. I know that she is going to be disappointed with that swim. I think about what I am going to say to her. This worries me. She looked so strong in the heat swim this morning, a contrast to her final swim this evening. I look down to the pool as Katie is exiting it; she looks like she has trouble pulling herself out of the water. She looks exhausted.

Katie dives into the diving pool to warm down. I decide that I will go and see her on pool deck where she is warming down in the dive pool. When I get down there, I see that she has her goggles on and her face is kind of tight in expression as if she is crying. I walk over to her.

Mandy: "Don't be disappointed Katie."

She looks at me and I can see that she is crying.

Mandy: "Give me a chance to go over the race, your taper, your warm up this evening, whether I set you enough warm down this morning and I will see where I went wrong."

Katie swims off, completing the rest of the warm down. I head back up to the grandstand. When Katie finishes her warm down and arrives back in the grandstand, I decide I really need to know what she is thinking and how she is feeling so I can tackle her disappointment the best way that I can. I hand her a questionnaire that has been devised by a colleague who is a psychologist. The questionnaire requires her to answer a series of 30 questions in relation to how she is feeling.

Mandy: "Katie, can you fill this out for me and hand it to me when you are finished. Can you also write down what you were thinking and feelings during your warm up, also while you were in the marshalling and throughout the race." 
I don't explain the questions to her as she is familiar with them, having filled it out before.

Mandy: "Katie, on a positive note, your turns have improved dramatically. Not once did you breathe in and out of the wall and your underwater work was superb. It is very obvious that you have been practising them in training - well done."

As Katie is filling out the questionnaire, I look to where I could have gone wrong with her preparation. Katie is so committed and she deserves a good performance. I want to see where I have let her down.

Have to fix this.

Have to make it better.

Mandy: "Katie, on Monday, can you book into the doctor and organise to have a blood test. I just want to make sure that there are no underlying health issues that we are dealing with that we don't know about." 


\section{CHAPTER FIVE}




\title{
The critique
}

\author{
"The unexamined life is not worth living \\ and the unlived life is not worth examining." (John Keats)
}

\section{Introduction}

This critique focuses on body pedagogies. Firstly, the experiences of three adolescent [myself and two others] swimmer bodies within the cultural context of Australian swimming and the body pedagogies [Act one and Act two] they were exposed to will be examined. Secondly, I focus specifically on the "durability" (Lee \& Macdonald, 2010) of body pedagogies in discussing the body pedagogies that myself and the two other participants now engage with some 10-30 years after being immersed in the culture of Australian swimming. Discussion explores the extent to which the body pedagogies that we were exposed to and participated in during adolescence are still being adopted (and legitimated), or alternatively, resisted and challenged. Analysis of data pursues the discourses of 'performance' and 'perfection.' While these two discourses provide the focus and structure for the analysis of the Acts, inevitably, some of the points raised extend beyond them. Analysis thus addresses the notions of exposure and effect of body pedagogies (see chapter 2) and illustrates the "durability" (Lee \& Macdonald, 2010) of body pedagogies occurring through discourses of performance and perfection within a sporting culture.

Inevitably a range of issues have come to the fore when examining the lived experiences of myself and the two other adolescent swimmers. I particularly sought to explore my own and the other participants' responses as adolescents to a wider (swimming) culture's compulsion to normalize body practices. Body pedagogies that became ethnophysiological in regard to our swimmer body (Jones, 2000; Jones et al., 2002, Jones et al., 2005; McMahon \& DinanThompson, 2008; Wright, 2000) were a key issue in this inquiry. The issues variously reflect dominant discourses and ideologies within the culture that we were a part of. From the stories told, discourses of performance and perfection 
seemingly pervade body pedagogies occurring in and through the Australian swimming culture. Secondly, amidst and embedded in these two discourses, the notions of technocracy, meritocracy, 'slim to win' are evident. Disciplinary power [panopticism, surveillance, differential power, technologies of the self, docile bodies, normalization] and ethnophysiology are also explored in this chapter. These concepts provide the means of interrogating practices, activities and social interactions [body pedagogies] that all relate to swimmers, coaches and others' understandings of the competitive and corporeal potential of the swimmer body. A further important feature of this critique is that the examination of the Australian swimming culture extends to the multiple sites [places] of practice described by Evans et al. (2008), which in this instance included the training environment, competition sites/events, and home environment, and encompassed social interactions with coaches, peers and family members.

Before embarking on the analysis of practices and discourses that are associated with the body pedagogics (Shilling, 2005) of the Australian swimming culture, it is helpful to revisit definitions of body pedagogies. Rich and Evans (2007) describe body pedagogies as "any conscious activity taken by persons, organizations, or the state designed to enhance individuals' understandings of their own and others' corporeality" (p. 41). They operate to "define the significance, value and potential of the body" (Evans et al., 2008, p. 17). Furthermore, "the pedagogies themselves, as well as the understandings of corporeality that they work towards, differ according to the time, space and place in which they are employed" (Cliff \& Wright, 2010, p. 222). For Shilling, body pedagogics (2005) classify bodies of value and status by defining and positioning those bodies deemed to be more or less valuable within specific social and cultural contexts. For Vigarello (1978, p. 9), “pedagogies are carriers of precepts which give the body a form, and control it to submit it to norms in a way more efficient than thought. The body is the emblem where culture inscribes its signs as blazons." Body pedagogies have been found to exist and 'operate' across and through multiple sites of practice, defining the significance and potential of the body in time, place and space (Evans et al., 2008). 


\section{Discourses of performance and perfection}

In my research, performativity and perfection emerged as the predominant discourses, consequently framing the critique for this research. Subsequently, I have utilized the term discourse to explain the range of messages, ideologies and beliefs that are perpetuated within each of these discourses. Foucault's (1979, 1984) use of the term 'discourse' is also drawn upon. Foucault uses the term 'discourse' to explain ways of thinking and speaking about aspects of reality - a formalised way of thinking that can be discernible through language, a social periphery defining what can be said about a particular subject. As Jones et al. (2005) have emphasised the processes of establishing and maintaining identities are inherently related to discourses. Thus the identities of myself and the other two participants in this research will be closely examined.

Perfection discourse [adapted from perfection code, Evans, Rich \& Holroyd, 2004] which is closely associated to the discourse of performativity, specifically relates to pedagogies including social interactions which have occurred based on the ideology that the body is imperfect and in need of being changed. Ball (2004) describes performativity as:

A technology; a culture and a mode of regulation, or even a system of terror that employs judgements, comparisons and displays as a means of control, attrition and change. The performances of individual subjects or organisations serve as measures of productivity or output, or displays of 'quality,' or 'moments' of promotion or inspection.

They stand for, encapsulate or represent the worth, quality and value of an individual or organisation within field of judgment. (p. 143)

When applying the notion of 'perfection' to the Australian swimming culture, the swimmer body is viewed as an unfinished product with the intention of amelioration [make or become better] occurring. In so doing, the discourse of perfection can be described as "structures of meaning defining what the body [in size, shape, predisposition and demeanour] is and ought to be; and how, for those who do not meet these ideals, there is treatment, repair and restoration" (Rich \& Evans, 2007, p. 44). Consequently, the data reveals how the swimmer bodies come to be managed, regulated, measured and compared against standards within 
the elite swimming culture. For the sake of 'slim to win's their results are subject to a wider technology which judges how well the swimmer body measures up [size/shape] to such standards (Rich \& Evans, 2007).

\section{Perfection}

The stories articulated by Mandy, Carly and I within the discourse of perfection reveal how our 'swimmer bodies' became adorned with cultural practices. In so doing, the stories reveal how our swimmer self and our desire to achieve competitive performance contributed to and reinforced the idea that a 'lean swimmer body is a fast swimmer body.' The hegemony of 'slim to win' (McMahon \& Dinan-Thompson, 2008) is a meme that was shown in this research to permeate the Australian swimming culture both at a club and elite level and has similarly been illuminated in the work by Jones et al. (2005). Swimming is a sport where there is no proven benefit (from a performance perspective) in seeking extreme thinness. Maglischo (1993) found no relationship between body shape and drag ${ }^{20}$ measured during actual swimming and found that even the most lean and tapered swimmers cannot remain streamlined enough to eliminate turbulence. Yet, as the stories in Act one and Act two showed, the belief that the slimmer or leaner a competitive swimmer becomes, the better s/he will perform (Drummer, Rosen, Heusner, Roberts \& Counsilman, 1987) remains strong and continues to persist in Australian swimming culture. Taub and Benson (1992, p. 77) say that "excess body fat and body weight in both males and females are widely considered by coaches, parents and participants [swimmers] to hinder performance. Yet, Australian Institute of Sport swimming physiologist David Pyne has highlighted that:

There are a few studies in different sports that associate better performance with lower skin folds. Actually there's not much data around on swimming, which is surprising given the emphasis on

\footnotetext{
${ }^{20}$ Drag can be described as a resistance that results from the space swimmers take up in the water, the shape they present to the water, limb movements that push water forward instead of backward, and the friction between the body and the streams of water that come in contact with it (Maglischo, 1993, p. 49).
} 
weight and shape by almost every coach. (Personal communication, January 19, 2007)

The bodily practices of weight monitoring and management may not be essential to performance; however acquiring the ideal shape is a notion that is deeply entrenched in the culture and continues to be recycled by coaches, team managers, swimmers and other social regulators (including parents and the media) as demonstrated by the stories told over a 30 year time frame. This in turn points to the "durability" (Lee \& Macdonald, 2010) of the ideology. Michael Bohl who is the current Australian swimming coach of the year spoke this year about the idea of 'slim to win,' describing how he engages with the ideology and engages with bodily practices for the sake of competitive performance. Discussing the performance of one of his female athletes, Bohl (2010) stated:

What happened after Beijing is that she really let her hair down. She put on maybe three or four kilos. Four kilos might not sound like much but if you think of a margarine container, there are eight of those you are carrying around and if you asked Michael Phelps ${ }^{21}$ to carry eight of them on his body and still line up and beat the best in the world, he'd think you were crazy. They get weighed and skinfolded every three or four weeks. (Forster, 2010, Australian Story transcript)

In contrast to Bohl's beliefs, Ian Thorpe ${ }^{22}$ commented:

The vast majority of coaches lecture about diet and they are obese, or they're criticizing commitment and they don't have the commitment that they should. When you get into the pool, it is like being 10 percent of your body weight. So that one or two kilograms that coaches stress over really equate to 100 or 200 grams in the water. I have swum well at 105 kilograms and I have swum well at 90, and that is a big range. (Fleming, 2005, Australian story transcript)

\footnotetext{
${ }^{21}$ Michael Phelps from the United States of America has won 14 Olympic gold medals and broken 37 world records in swimming.

${ }^{22}$ Ian Thorpe from Australia won five Olympic gold medals, broke 22 world records throughout his career, won six gold medals in one World Championship event and was named World swimmer of the year four times.
} 
Previous research on disordered eating has revealed that the pressure placed on athletes by their coaches was influential in the onset of such behaviour (Griffin and Harris, 1996; Jones et al., 2005; Patel, Greydanus, Pratt \& Phillips, 2003; Sungot-Borden, 1994). The culture in which the swimmers were immersed was a site of social practices "characterised by pre-occupation with conformity, management and control over the body" (Garrett, 2004, p. 143). The emphasis placed upon leanness arguably reflects both the dominance of particular social ideas and values and the power hierarchies that sustain that dominance and the associated pedagogical practices.

Within the discourse of perfection all three of us were involved in some form of monitoring of our bodies and eating by coaches and/or managers motivated by the idea of 'slim to win.' We were taught how to view and experience our swimmer bodies in particular ways (Jones et al., 2005). Mandy, Carly and I did not question the ideas of 'slim to win' as we all compliantly engaged in ethnophysiological tests, body practices and training for the sake of perceived competitive performance. The pressure we felt was evident in all three of our internal dialogues as we tried to conform to 'slim to win.' This was hegemonically enforced both through the cultural value of such a body and the coaches' knowledge that 'slim to win' improved competitive performance (Johns \& Johns, 2000; Wright, 2000). Our voices exemplify the pressure that we felt to conform to the ideal swimmer body, particularly through the daily body practices that we were all exposed to.

Carly: "These scales are wrong! I am 38 kilograms. They have to be wrong! I was 38 kilograms before I left."

Carly (internal feelings): A sense of fear overcomes me because of my weight.

Carly's coach: "If you put on weight you will get your periods, then your body will change and you will swim slow."

Carly's (internal dialogue): I hope I don't get my periods now that I have put on weight. Please God, don't let me get my periods! I won't swim fast anymore if I get my periods. 
Jenny: Two days in a row, my weight has increased by 0.15 of a kilogram. I remember drinking water on the bus on the way to the pool. You idiot, that water could affect my weight! Maybe I have just sweated some of that water out during the session? What happens if that is not enough? Go to the toilet Jenny. That could help.

Jenny: Am desperate to lose weight.

Mandy: My heart sinks. I cannot understand why I would have had a weight increase. I have been so careful, just can't understand it. As a method of self-protection, I withdraw into myself.

For Carly, the measurement and assessment of her swimmer body first occurred when she was ten years old. Under the direction of her coach, the dietician assessed her body, and also demonstrated an act of comparison by informing her of her percentage body fat in relation to the readings of a senior female team mate. In this interaction Carly learnt that the lower percentage body fat that she had, the faster that she would swim, which in so doing exemplified Bernstein's (2000) notion of a 'pedagogical device.' When Carly was 11 years old, she was selected in an age group development training camp to be held interstate, travelling without her coach and family. At the end of this camp, Carly was required to have her weight and skin fold measurements recorded by a coach on the camp. The practices of measure and assessment, driven by the notion of 'slim to win' were familiar to Carly, having been first exposed to it 12 months earlier by her own coach.

I was not subjected to the same weekly body measurement and assessment scrutiny by my own coach; however as a member or the Australian swimming team, I was subjected to daily weigh-ins as well as having skin fold measurements taken every five days. Our swimmer bodies were followed closely by coaches and managers and having a reading that was higher than the recommended total skin fold measurements [a total of measure of 70 arising from measurements taken at 7 
sites $^{23}$ ] or having an increase on the scales held ramifications [i.e., threatening to be expelled from the team].

Like Carly, Mandy was subjected to weekly weigh-ins by her own coach. However, like my own experiences on the Australian representative swimming teams, she was also subjected to daily scrutiny of her body and its measurement. When she recorded a weight increase, the punishment of restricted food at meal times was enforced for what seemingly appears to be her body's failure to conform. The place where Mandy could consume her meals was also restricted as after her weight increase Mandy was only allowed to eat her meals at the same table as the coaches, who consequently had enforced the punishment. The extent of the surveillance of Mandy during meal times is detailed below when discussing surveillance and panoptocism.

From the bodily practices implemented by coaches, physiologists, dieticians and team managers, our adolescent swimmer bodies with a series of problems which needed "negotiation by working representational transformations that can render them nearer to or further from the bodily appearances of their peers" (Prout, 2000, p. 5). These bodily measurements were not conducted in private but rather, occurred in a public domain [i.e., on pool deck, in a hotel room filled with people], with swimmers often lining up like a production line of a factory to have their bodies monitored through testing regimes. Subsequently, due to the lack of privacy, all three of us at different times when having our bodies measured and tested were privy to each others' body measurements, as the 'fat numbers' of others were revealed. Carly and I subsequently tried to better our fat numbers within this context thus engaging in a competition to possess the lowest fat numbers of the females being tested. Below are some of the interactions which presented our swimmer bodies with a series of problems, which were subsequently, were acted upon, assimilating to expectations of those around us.

Measuring lady (to Carly): "Your percentage body fat is seven percent. You're as low as Katie."

Carly's response (internal): I am going to beat Katie next week.

\footnotetext{
${ }^{23}$ Seven sites of skin fold tests included measurements taken from tricep, bicep, subscapular, front thigh, iliac crest, medial calf and abdominal.
} 
Male coach (to Carly): "These scales say that you are 46 kilograms. They are not wrong."

Carly's response (internal): I notice that some of the other swimmers in the line are listening intently to what my weight is, my response and also the coach's response. I am going to be in so much trouble. I am fat. I cannot believe I have a weight problem. I am terrified of what my punishment will be. My coach does not like us girls to be heavier than 45 kilograms. I am going to have so much running to get back to a weight under 45 kilograms.

Manager (to me): "Jenny, we are concerned with your weight increases. You need to think about whether you still want a place on the team because your weight is indicating that you are not remaining focused and committed. Your skin fold readings show us that you are losing fitness. We will be interested to see how you go in your skin fold measurements tomorrow?"

My response (internal): At dinner that night I hardly eat. I am really worried about my weight. Have to fix my body.

Physiologist (doing skin folds on another swimmer): "14."

My response: I wonder what site that reading was taken from. Who is getting measured? Is it one of the female swimmers? I stand on the tips of my toes and can see that it is one of the girls but cannot make out who it is. Was it taken from her thigh? Heck, five days ago my thigh measurement was 21. Hate my body. It is such a failure. Feel vulnerable. Need to fix it.

Physiologist (doing skin folds on female team mate): Physiologist: "61 overall, great work."

My response (internal): None of my measurements are ever under a reading of ten. I can't believe that she just got 61 overall. I am going to get into so much trouble from my coach. I know that I am going to be well over 70. Need to fix my fat numbers. Need to fix my body.

Coach (to Mandy): "55 kilograms. You have put on two kilograms." 
Mandy's response: I stick my fingers down my throat and as I do I think about the body that I need to have, a body that will be accepted by the coaches. My dinner comes up. The fruit burns as it comes up. This is going to fix everything.

When differences [in weight and skin fold measurements] were brought to our attention, we responded compulsively. We wanted to transform our swimmer bodies so that they aligned more closely with both measurements of peers (Prout, 2000) and with the expectations of coaches and management. Both Carly and I highlighted ways in which skin fold readings, weight measurements and food that were deemed 'bad' became the focus for interactions, emergent identities and unhealthy relationships that were formed with our swimmer bodies. Below extracts from internal dialogue reveal how our swimmer identities started to become fractured [damaged]. Our 'fractured bodies' (adapted from fractured identities; Wainwright et al., 2007) exemplify a wounded body/self revealed through our internal dialogue.

Jenny: I hate my body. Am such a loser. It is such a failure.

Carly: I shouldn't have eaten the ice cream. I am such a fat pig.

Carly: I am so fat and disgusting.

Carly: Please God; don't let me get my periods! I won't swim fast anymore if I get my periods.

Carly: My fat is so disgusting.

Jenny: Need to fix my body!

Jenny: Why are you letting me down this way?

Mandy: Have to do something - can't let it let me down anymore

Notably, some of the internal dialogue positions our swimmer body/s in the third person, representing a seemingly dislocated body and self, a point I return to below. Subsequently, it was the bodies that skin fold tests, and weigh-ins deemed as 'less than perfect swimmer bodies' which impacted upon the relationship we had with our bodies and damaged our swimmer selves. Similar to previous 
research conducted by Shilling (2005), Grosz (1995), Prout (2000) and James (2000), the internal dialogue of myself, Carly and Mandy provides insights into the connections between corporeal bodies as agentic entities, bodily experience and culture.

For myself, Carly and Mandy, practices of comparison, measurement and assessment occurring through pedagogies within the training environment and in team situations exemplified acts of corporeal perfection (Rich \& Evans, 2007). Hall (1996, p. 6) states, "identities are created through the successful chaining of the subject to the discourse." Thus, the perfection discourse and memes detailed above and located within the cultural context of Australian swimming dictated what we thought of ourselves and what we felt about ourselves. For Carly, Mandy and myself, the discourse of perfection certainly influenced our ideas, body practices and subsequently, after not 'measuring up,' we developed 'fractured swimmer bodies.' Wilmore and Costill (1992) revealed that athletes who are immersed in a highly competitive culture have increasing amounts of pressure placed on them to manipulate their eating and weight in order to maximize their performance. Consequently, for me, the discourse of perfection and my attempt to conform to the ideal swimmer body [perfection] became an obsession. I sought help from those within and outside the elite swimming culture (i.e., sports medicine doctor/general practitioner and fellow team mate) to adhere and conform to the expectations of/for my body. The sports medicine doctor and general practitioner seemingly accepted the discourse of bodily perfection without question, prescribing me medication [thyroid medication, prescription speed] that in turn affected my functioning in life and training. A senior female team mate also assisted me by showing me how she conformed to the expected bodily ideals by purging after meals. Dissatisfaction with my body was clearly evidenced in my internal dialogue and self-regulatory practices.

Jenny: I cannot look at myself.

Jenny: I am ashamed of what I look like!

Jenny: Why can't I achieve the swimmer body that I need for Olympic success? 
Mandy's story was similar to mine, where another female team mate introduced her to purging after meals. Dissatisfaction with her swimmer body was similarly apparent through her internal dialogue and self-regulatory practices.

Mandy: Have to do something - can't let it let me down any more.

Engaging with prescription drugs and purging after meals caused us to engage with a 'risk culture' (Howe, 2004) while striving for body perfection. Howe (2004) describes a risk culture as "an elite sporting culture that may be seen as a dome that entraps health and positive encounters with the body" (p. 183). Consequently, our health became compromised with the use of diet drugs, an act motivated by my desire for bodily perfection. Rich (2010, p. 149) echoes Howe's (2004) sentiments of 'risk culture' referring to such behaviour "as junctures where conflicting pedagogical knowledge and regulatory aspirations of health clash." Yates, Shisslak, Crago and Allender (1994) revealed that disturbed eating behaviours were found to occur in sporting cultures that emphasized leanness, particularly where the athlete's scantily clad body was on display.

\section{Performativity}

The performativity discourse [adapted from performance code, Evans, Rich \& Holroyd, 2004] places importance on productivity. Using Foucauldian language, discourse has been utilised accordingly to thus describe the site of high performance sport that uses technologies of discipline to produce performing athletes (Tsang, 2000). When applied to the Australian swimming culture, it relates to pedagogies, interactions and decisions that focus on and serve to regulate and monitor the output of the swimmers. It hones in on the specialised skills necessary for the production of the specific output, which in this case, is competitive performance.

The stories of Carly, Mandy and myself showed that through the implementation of practices and entrenched 'memes' [ideas, symbols or practices], Australian swimming coaches, managers and swimmers demonstrated technocentric ideologies as our 'swimmer bodies' were "viewed as an instrument and object for manipulation" (Bain, 1990, p. 29) in order to achieve competitive 
performance. In the amateur (as told by Carly) and elite (as told by Mandy and Jenny) contexts of the Australian swimming culture, the overall focus of coaches, fellow team mates and others immersed in the culture was shown to be extrinsically based on the achievement of competitive performance. This was evidenced through lived experiences and also the swimmers' complicity in assimilating to the ideal swimmer body and the bodily norms. Bain (1990) explains "that within such a technocentric ideology, people are viewed as human resources where attention is focused on the development of an increasingly effective and efficient means for achieving goals" (p. 29). In this instance, the three swimmers' bodies were essentially regarded as swimmers to be trained to produce competitive performances. The coaches and team managers saw our 'swimmer bodies' as objects "to be slenderised and toned in order to increase [their] value" (Bain, 1990, p. 30). Implementing regular weigh-ins, skin fold tests as well as training procedures that encompassed lactic acid ${ }^{24}$ tests, heart-rate tests $^{25}$ and physical challenges which occurred via the 'micro-practices' (Dowling-Naess, 2001; Foucault 1977) of everyday practice. The practices and interactions, from coaches, team managers and others [peers, family members] reveal how our swimmer bodies became a "site of explicit exposure" (Pillow, 1997, p. 349) reinforcing Australian swimming’s 'body norms' (Ellingson, 2006).

Performativity has been found to persist in discourses of competition and achievement (Evans, Davies \& Wright, 2004), training discourses and in team situations within the Australian swimming culture. Within the performativity discourse; the coach is the fundamental key to performance occurring and subsequently becomes the overseer of body regulation and monitoring for the sake of achieving competitive outcomes. For Carly, Mandy and myself, team coaches and to a lesser degree, team managers, became the ultimate overseers. Others, and particularly, the parents of Carly and myself also took on the role of overseer, bringing performance discourses from swimming culture into the home environment. As Lopiano and Zotos (1992, p. 278) state, coaches are perceived as

\footnotetext{
${ }^{24}$ The lactic acid test is used by coaches to determine the lactate threshold of a swimmer which subsequently determines what distance the swimmer will perform best at. The lactate threshold is defined as the point when lactic acid [the burning, heavy sensation during intense fitness] starts to accumulate, some testers approximate this by using the point at which lactate reaches a concentration of $4 \mathrm{mM}$ (at rest it is around $1 \mathrm{mM}$ ).

${ }^{25}$ A heart rate test is used by coaches who are interested in monitoring their swimmers' heart rate to gain maximum efficiency from their training which in turn leads to competitive performance.
} 
"having the key that will unlock the secret to successful athletic performance" and the stories told by Carly, Mandy and myself illustrate how for the sake of our improved competitive performance, we willingly accepted the ideas and practices of coaches. In so doing Carly, Mandy and myself co-contributed to the legitimation and normalisation of the performance discourse. However, the subjectification of practices for the sake of performance is what Foucault refers to as "meticulous rituals of power." It "manifests the subjugation of those who are perceived as objects and the objectification of those who are subjugated" (1977, p. 185). For Foucault, a central element of performativity is the production of intelligible and useful bodies which within the discourse of performance can be referred to as performing bodies. The practices to attain performing bodies described by Carly, Mandy and myself were, in Foucault's words, "the target for new mechanisms of power" that are "offered up to new forms of knowledge" (1977, p. 155). Below, extracts show how Carly and Mandy accepted their coaches practice, interaction and punishment [Carly] willingly. They unquestioned their punishment and internal dialogue reveals their complicity for the sake of competitive performance.

\section{Carly}

Carly's coach: “Carly, 35. Carly, if you don't hold 34's, I am going to make you repeat this set at the end of the session."

On the fourteenth lap, I touch the wall and lift my head to hear my time.

Carly's coach: "Carly, 35. You are making this set up at the end of the session."

I am disappointed in my times and myself but I know I am putting everything into this set. At least by repeating this set after everyone is out of the water, I am putting extra money in the bank, putting in extra than my competitors would be which will in turn make me swim faster. However, I feel somehow hard done by as I realise, that half of the other swimmers aren't making their times either but I am the only one that has to stay back and repeat it. I wonder why I am the only one that always has to stay back and repeat sets. It isn't fair. I feel singled out, picked 
on. I am presented with a quandary between being hard done by and knowing that this is actually good for me so I say nothing to my coach and accept it obligingly.

\section{Mandy}

Mandy: "I can't go any further."

Bob: "If you don't keep reducing your times, we will keep going until you do."

My shoulders drop under the water. Instead of feeling inspired, I feel deflated, demoralized. I feel like getting out of the water but I know that my coach has produced champions in the past and I have to do what he says if I want to be the best. I push off the wall for my third mile and my body is aching, really hurting. I cry as I swim and I try desperately to suck the oxygen in because I am puffing so much from the exertion. I finish off the final two miles, descending them as my coach stated, avoiding repeating the entire set. I am still crying. Without warning, my body starts to convulse and I vomit up the content of my stomach. He sees me but doesn't say anything to me, nothing about my exertion, about me achieving his expectations. At the end of the session, I climb out of the pool. I don't feel a sense of achievement, but instead feel beaten, affected by my coach's words.

Mandy and Carly's stories exemplify how their coaches were the overseer of their training and performance and their gaze subsequently critiqued their swimmer body performance, which is also inherent through the concept of Foucault's (1977) panopticism. Mandy and Carly's awareness of this overseer, as described by Tsang (2000) is a gaze which acts as power, or a constraint of action as they conform to such practices (Foucault, 1977). Shogan (1999) described such practice or constraint of action as technologies of discipline. Further, she describes this as constraints on action that circumscribe space, time and modality of movement with the intention of producing skilled athletes. As illustrated above, Mandy and Carly both obligingly accepted the punishment or the threat of, when their training performance did not comply with the expectations placed on them by their coach.

The concept of producing the skilled and competitive swimmer body pervades the performative discourse of Australian swimming. Shilling (1993) 
explains how the body in modern culture has become the source of physical capital, "the possessor of power, status and symbolic forms" (p. 127). The physical capital inherent in our swimmer bodies brought reward in the form of social privilege, as the more competitive our swimmer body was, the more social privilege that we were afforded. Consequently, a lack of physical performance was shown to engender social isolation. Below, some extracts from Carly and myself exemplify social privilege and isolation.

\section{Carly}

I climb out of the diving pool and put my tracksuit on. I head back up to the grandstand where I know my coach will be waiting for me. I take my time as I am still trying to make sense of my poor form. My eyes scan to where my club is sitting. I walk towards them. One of the senior female swimmers in my club has just spoken to Tom and is heading down for her race. Her eyes connect with mine and our shoulders nearly rub as she walks past me but she says nothing. Her face is expressionless. This is not out of character for her as she has ignored me in the past when I have not swum well. She tends to behave in the opposite when I am swimming well. I walk towards my coach, he is only metres away. His eyes connect with mine. I stop when I get a metre away from him. He pauses, looking at me. He then raises his left hand and he places it onto my right shoulder. It is only there for a few seconds before he takes it away. He then turns his body away from mine and focuses on the race that is underway. I am expecting him to say something, instead he says nothing. I wonder if that is it.

Is he finished with me?

I wait momentarily, just in case it isn't. But then I realize that his interaction with me is over.

\section{Jenny}

The final swimmer has split around 55 seconds. I know what this means. I walk back with my team mates to the warm down pool. A coach and team manager is waiting for us. We all gather around the coach who has our splits written on the program. It is now clear that I am the slowest. Nobody says anything to me. They don't even officially tell me that I am going to miss out on the final tonight. They 
just assume that I would know which I do. I dive into the warm down pool and cry.

Am such a failure.

After the warm down, I get out of the pool and get changed. I head back up to the grandstand where the rest of my team mates are sitting. When I arrive to where Australia is sitting, nobody says anything to me, not the coaches, not the managers and not even my team mates. I sit there alone.

\section{Jenny}

It has been eight months since I have competed-eight months since the biggest failure of my career, where I failed to make the Australian Olympic team. I walk into where the warm up is. I see a lot of familiar faces. I smile at a coach, one that I have been away with on Australian teams. He is a coach that I worked with on the 1991 World Championship team as my own coach had been placed with the long distance swimmers. We worked well together. As our eyes meet, he turns away without so much of a smile or acknowledgement.

Did he purposely turn away or didn't he see me?

I sit down and stretch. A group of four female swimmers from another club approach me. Three of them I have been on Australian teams with. I smile at them, an invitation, or so I thought for them to come over. They giggle and whisper to each other and keep walking.

A culture that engages with the discourse of performativity has swimmers that have "lost their claim to respect except in terms of performance" (Ball, 2004, p. 4). This not only determines the relationships between the athlete and the coach but also between other peers and family members, but more profoundly in relation to their swimmer [corporeal] self as exemplified through the data presented.

When critically examining the notion of 'physical capital' further, I take up Bourdieu's (1984) conceptualisation of physical capital as not merely an embodied capacity to use the body, but also, capital in terms of the appearance of the body, the body as evidence of particular work, and in this case physical fitness of the body in terms of perceived competitive performance. The latter notion of 
physical capital was embedded within the discourse of performativity within the Australian swimming culture. Our swimmer bodies and their appearance were assumed as an indicator of our physical fitness as well as how well we were able to manage our swimmer bodies. A swimmer's level of fitness was often judged by coaches, managers and even swimmers [including myself] purely on bodily appearance, what the body looked like it could do before witnessing what it was able to do. The assumption that the lean swimmer body equates to competitive performance is limited in terms of appearance being used as the basis to judge fitness, which is subsequently equated to performance potential (in terms of prospective times). In so doing, the complexities of performance and the multitude of factors impacting upon and contributing to performance are arguably denied.

The extracts below exemplify how coaches, team managers and even myself assumed that performance was dependent purely on the aesthetic attributes of the swimmer and reminds us of the power attached to the notion of 'slim to win.'

Carly's coach: "How could she put on so much weight in ten days? What did they do to her down there? Look at how unfit she is. I should never have let her go. It was a mistake. This will be the end of her if I don't watch her weight closely."

Australian swim team manager: "Jenny, we are concerned with your weight increases. You need to think about whether you still want a place on the team because your weight is indicating that you are not remaining focused and committed. Your skin fold readings show us that you are losing fitness. We will be interested to see how you go in your skin fold measurements tomorrow?"

My response (internal): I begin questioning my level of commitment.

Jenny: I look over to my competitors standing beside me in the other seven lanes ready to start. They are tanned, toned and fatless. My body stiffens with anxiety. I know what this means. They are going to swim fast.

My internal dialogue and also my own actions of observing a fellow competitor revealed what O'Brien, Martin Ginis \& Kirk (2008) refer to as 
“objectifaction" (p. 117). O'Brien et al. (2008) define objectification as "the experience of being treated as an object to be looked at and evaluated, solely on the basis of physical appearance." They explain that the observed "come to think about and value their bodies from an observer's perspective, focusing on what their bodies look like rather than on unobservable body attributes such as physical abilities" (p. 117). I began questioning my own level of commitment when my body did not measure up to the observer's perspective (i.e team managers) and in so doing engaged in "self-objectification" (O’Brien et al., 2008, p. 117).

Extraordinarily, I also engaged in "objectification" when I valued the body of a fellow competitor on her physical appearance, assuming competitive performance would be achieved from the aesthetic appearance of her swimmer body.

Just as I sought outside help, Carly and her family did the same. In this case it was on the advice of her coach, to enhance her competitive performance. At 13 years of age, Carly's mother took her to a sports medicine doctor who in turn gave her a bag of tablets. Carly took the tablets as per the directions believing that they would give her a competitive edge. The seeking of outside help for the sake of competitive performance relates to what Evans, Rich \& Holroyd (2004, p. 136) term "acquired resources," accessed and used all in the name of performance and to have an edge over competitors.

\section{Reproduction of the discourses of perfection and performance: the sites of learning}

The reproduction of the discourses of perfection and performance can be identified though the examination of sites where the learning has occurred. Notably, Bourdieu (1991) identified two principal sites responsible for the reproduction of dominant discourses; the family and the educational system. In this research, while coaches, team managers and other swimmers were identified as the primary sources of knowledge relating to [bodily] perfection and performance, the importance of the family was also revealed as a critical factor (and site) in the perpetuation of practices and beliefs relating to [bodily] perfection and performance. For both Carly and myself, family were key to the reproduction of these discourses. Although Mandy did not undergo the same 
scrutiny in her home environment, it did occur in her training environment and in team situations. The extent of 'slim to win' is realised when, for Carly and myself, there was no escaping the ideology and practices that surround it. Within and beyond the immediate site of swimming, we were immersed and became entrenched with the notion, social interactions and subsequent practices of "slim to win.' Previous research (Brownell, 1991; Rodin \& Larsen, 1992) revealed how elite athletes have been identified as a group who are subject to intense pressure to conform to an ideal body shape. The dominance of the discourses of performance and perfection was revealed through the data as pervading not merely interactions between us and our coaches (in both amateur and elite sporting contexts), but also those with our peers and family members. Furthermore, the data also highlights the various ways in which the discourses of [bodily] perfection and performance have permeated speech and practices of swimmers' bodies over a period of 35 years. The durability (Lee \& Macdonald, 2010) of the discourses is striking revealing the extent of corporeal internalization and the centrality of body techniques to the habitus [a set of dispositions which generate practices and perceptions] (Lee \& Macdonald, 2010).

\section{Disciplinary Power}

Disciplinary power is instigated through technologies of dominance, which includes classification, regulation, normalisation and surveillance. Sports scholars have applied the technology of surveillance to explore how athletic bodies become compliant and productive (Aycock, 1992; Carlisle-Duncan, 1994; Chapman, 1997; Maguire \& Mansfield, 1998; Markula, 1995). Acts of disciplinary power pervade body pedagogies occurring within sporting cultures, particularly Australian swimming because as Evans et al. (2008, p. 98) state, "all pedagogical relations are power relations; there is no instruction without regulation; no pedagogy is divorced from control." Menzies ${ }^{26}$ (2005) revealed the power exerted by some Australian swimming coaches:

\footnotetext{
${ }^{26}$ Tracey Menzies is an Australian swimming coach, most known for coaching five-time Olympic champion Ian Thorpe, Australia's most successful Olympian, from late 2002 until his retirement in 2006.
} 
There are coaches that still use those dogmatic approaches where they own the athlete, they own their eating rights, they own their training rights, they own even their thinking. They're dictators. I don't see that it's a successful way of coaching. (Fleming, 2005, Australian story transcript)

Concepts of power are particularly pertinent to this research and subsequently this critique. For Harvey and Sparks (1991), discipline is a collection of modern methods of domination which is integrated through social order that aims to disperse, mark and repair bodies, training them so their movements and gestures provide an optimum efficiency. In this research, coaches and team managers, revealed through the stories of experience by Carly, Mandy and myself, exercised disciplinary power (Foucault, 1977) by 'normalising,' 'regulating' 'classifying' and 'surveilling' the swimmer body for the sake of performance goals.

The regulatory physiological practices described by Carly, Mandy and myself were 'ethnophysiological' as they were triggered within the social context of Australian swimming and legitimated through "values packaged in a scientific wrapping" (Vertinsky, 1985, p. 73). Daily weigh-ins and skin fold tests revealed by all three of us were tactics exemplifying "exertion of maximum social control with the minimum expenditure of force" (Foucault, 1977, p. 136). At a club (amateur) level and also National level, the data points to Australian swimming culture representing an institution and site where particular techniques of power became concentrated and were brought to bear on the three individuals in systematic ways.

Foucault's (1977) mechanism of power, where bodies embody conformity to normative behaviour and as such become obedient and docile, is clearly pertinent to the data presented. Foucault explained that power in its capillary form of existence, "reaches into the very grain of individuals, touches their bodies and inserts itself into their action and attitudes, their discourses, learning processes and everyday lives" (Foucault, 1977, p. 39) thus revealing the potential impact of culture. From a Foucauldian perspective, disciplinary processes such as the training regimes where the coaches and team managers were the overseer of bodily practices and training, in turn produced a disciplined and docile body, a 
body that was obedient "subjected, used, transformed, and improved" (Foucault, 1977, p. 136). All three of us obligingly engaged in bodily practices as well as training regimes for the sake of bodily perfection and competitive performance.

Subsequently, all three of our swimmer bodies became docile, compliant and productive through the practices and training regimes implemented by coaches, managers and our selves within the Australian swimming culture. Through practice, our swimmer bodies were controlled and monitored which “imposed a relation of docility-utility" (Foucault, 1975, p. 63) for the sake of performance. Our bodies became the subject of technologies of power, ascertaining that as swimmers, we were "enmeshed in a web of power created by discourse, which operates within the daily exchanges between individuals (the coaches/managers/fellow team mates/selves [sic]), groups and institutions" (Foucault, 1977, p. 136). Their practices were promoted through the concepts of productivity, efficiency and performance which were inscribed in the swimmers, thus becoming normative within the culture. Foucault (1991, p. 199) summarised that "the existence of a whole set of techniques and institutions for measuring, supervising and correcting the abnormal" resembles the assimilation bodily practices that we experienced within the Australian swimming culture. Thus, this is "architecturally representative of the panopticon" (p. 200).

Foucault's (1977) work on disciplinary power, specifically how disciplinary techniques regulate the movements of individuals is particular pertinent to Mandy and her story from Act two. After being verbally abused from her coach in regard to her performance, Mandy internalised her anger, silencing herself from responding to his verbal and public outburst. Mandy's response, or lack thereof, can therefore be understood as a form of docility, exemplifying the control and power that her coach had over her. In a similar situation Denison (2007, p. 376), explains how the coach becomes an "agent of normalization" and thus contributes to the docility of athletes, which in this case was Mandy.

Foucault used the notion of docility to demonstrate how bodies become manipulable and effective means for discipline. When docile, the body becomes useful as it can be moulded [discourses of performance and perfection] as a vehicle for the technologies of domination. (Markula \& Pringle, 2006, p. 74) 
It can be assumed that in an act of domination, Mandy's coach wanted her to mould successfully within the discourse of performance, regarding that as the key to enhanced competitive performance.

\section{Panoptocism}

An important feature of disciplinary cultures is what Foucault (1996) calls "the problem of total visibility of bodies, of individuals and things, under a system of centralised surveillance" (p. 226). As adolescent swimmers, most of the stories of experience told by Carly, Mandy and myself in Act one and Act two fell upon the backdrop of the pool. Rinehart's (1998) research into youth swimming identified the pool as a surveillance mechanism that was able to produce trained, efficient and obedient bodies. Accordingly, panoptocism occurs in places where people are watched and trained for optimal functioning. Foucault (1977) used the metaphor panopticon to articulate the operations of power and surveillance occurring in contemporary society.

In considering the Australian swimming culture within which myself and the other two participants were immersed, the metaphor of the panopticon offers a useful way of understanding the power relationships occurring between us the coaches, and sometimes managers and family. It exemplifies how social control was administered without coercion. The stories of experience shared by Carly, Mandy and myself exemplify panoptocism as our coaches and/or team managers, through their instruction and implementation of practices were either situated in the grand stand [competitions] or standing above on pool deck to us swimmers below, thus maximising their visibility of our bodies and performance. The coaches, team managers and in some instances family members, doubled as the prison guard, as described by Foucault (1977) when referring to Bentham's prison - holding the position of power, controlling us [Mandy, Carly and I], utilising disciplinary power by overseeing our training regimes and practices relating to [bodily] perfection and performance.

It was Foucault's belief that power is more effective when it is hidden from view (Danaher, Schirato \& Webb, 2000; Lang, 2010). While the actions of the 
coaches and/or team managers were not violent, they were invasive and controlling, functioning at the body level of our swimmer bodies, critiquing its performance. Disciplinary tactics occurring within the panopticon of the pool environment made power invisible, while maximizing the visibility of each subject [the swimmers]. Thus, our bodies became a site of "explicit exposure" (Pillow, 1997, p. 349), revealing disciplinary mechanisms of surveillance.

Panoptocism represents an ideology of culture that makes explicit the ways in which surveillance and self-policing are utilised to ensure order and control. Below, some extracts from the data exemplify how control was administered without coercion and illustrate the implications [our swimmer response] of this non-violent control. The data highlights the power of surveillance and how the ever observing gaze of coaches and others [mother] became a powerful form of control.

Carly: Everyone's eyes are fixed on me, most of all my coach. I am so embarrassed of what I have done to my body.

Response: I am doing everything that I can to lose the fat. I am hardly eating.

Jenny: My consciousness is alerted to the team managers' gaze. They are looking to see what is on my plate.

Response: I feel guilty for eating. Their constant gaze suppresses my appetite, creating a false sense of fullness. Somehow my stomach has been tricked into feeling satisfied, however I have only eaten three spoonfuls of food.

Mandy: The eyes of the coaches fixate on me, watching me as I put the fruit into my mouth.

Response: Ifeel self-conscious and stop eating.

For Foucault, disciplinary power which was once executed in the form of physical domination through the use of firearms and material constraints over the body is no longer required as it could be achieved through isolation and "a gaze an inspecting gaze, a gaze which each individual under its weight will end by 
interiorising to the point that he is his own overseer with each individual thus exercising this surveillance over, and against" themself (Foucault, 1977, p. 155). The stories reveal the power of the gaze. Our swimmer bodies were inescapably subjected both to our own and others' evaluative gaze occurring in various sites including training, competition and home and even to the extent where the three of us began to gaze on each other. Germov and Williams (1999, p. 125) refer to the concept of gaze utilized upon the self and others as "body police." They describe this as the "internalisation of the cultural imperatives of the thin ideal where one is encouraged to modify and monitor themselves and others in a neverending process of body surveillance to conform to the culturally accepted body." We saw our swimmer bodies as failing to meet the expectations of 'slim to win' permeating the Australian swimming culture. We became our own "body police" (Germov \& Williams, 1999) as we became critical of not only our own but others' swimmer bodies. When on representative teams, Mandy and I revealed that the gaze intensified and subsequently multiplied as it was no longer confined to the singular authoritative gaze of their coach but included, other coaches, team managers, spectators and other swimmers.

\section{Self surveillance}

The gaze and surveillance over Mandy and my bodies led us to seek out other measures, in an act of conformity and assimilation, thus striving for the lean and fast swimmer body. Mandy saw purging as an act of maintaining the ideal body but for me it was sought out in response to the threat that I was going to be expelled from the team. These 'other measures' were introduced to Mandy and myself by a female team-mate, resembling Foucault's technology of self (Foucault, 1997). With the assistance of a fellow swimmer, we learned and engaged in purging practices which "permitted them to effect by their own means [sic], with the help of others, an operation on their own body [sic] so as to transform themselves [sic] in order to attain a certain state of perfection [sic]" (Foucault, 1997, p. 225).

Further, in various ways as swimmers we somewhat transformed as we interiorized and embodied the surveillance, gaze and ideas of others until they 
eventually began "exercising surveillance over themselves" (Foucault, 1977, p. 155). Mandy and myself became our own overseer, as we "exercised the same surveillance over and against” ourselves (Foucault, 1977, p. 155). We purged our bodies and severely limited food intake in response to the gaze, and in an endeavour to meet 'the ideal physique' set by managers and swim coaches and the 'slim to win' ideology. In turn, the gaze became an effective formula for coaches and managers, "as it was exercised continuously, for what turned out to be minimal cost" (Foucault, 1977, p. 155) and so it was that it became possible for them to "qualify, classify and punish" (Foucault, 1977, pp. 184-185) us when our bodies failed to meet their expectations.

Subsequently, I became trapped in a vicious cycle, where conforming to the perfect swimmer became tied to my self-worth. Responsibility for my body was placed on myself by others (Featherstone, 1991) as I was seen as uncommitted if my body was not conforming. I adopted extreme self-regulatory practices in the belief that I had control over what sort of body shape I could acquire. Bartky (1988) on bodily shame states how it is "related to the extent to which they have internalised standards of bodily acceptability" (p. 68). My stories evidenced a belief that I did not measure up within the discourses of performance and perfection in the elite swimming culture as I did not possess a body deemed representative of Olympic success. In turn, my self became defined by my ability to achieve perfection and subsequently performance. Drug usage was complicit with destructive self-regulatory behaviour. Australian Institute of Sport physiologist, David Pyne has stated performance issues can be related to body issues in Australian swimming culture.

As a physiologist, my research has been more on the analysis and performance issues, but all that can go out the window if the swimmer/athlete is distracted with body, image issues, anxiety and/or an overbearing coach. It is unfortunately a scenario that we have dealt with on occasions in the past. (Personal communication, January 23, 2007)

Ironically, it was the distraction with obtaining the perfection swimmer body [slim to win] that led to my athletic and personal demise. 


\section{Technologies of the self}

Foucault (1988, p. 18) defines technologies of the self as processes whereby individuals which:

Effect by their own means or with the help of others a certain number of operations on their own bodies and souls, thoughts, conduct, and way of being, so as to transform themselves in order to attain a certain state of happiness, purity, wisdom, perfection, or immortality.

Foucault's (1988) notion provides an insight to the individual as both the object and subject of power offering an explanation of how human experience is shaped by relations of power. In this analysis, Foucault (1988) examined how individuals involved in power relations [body pedagogies] learned to recognize the discourses of knowledge and in so doing how they acted upon this knowledge, and how they came to understand and recognize themselves as humans through this particular discourse (Markula \& Pringle, 2006). Thus, technologies of the self are about identifying the actions of an individual within discursive power relations.

After a long period, I was finally able to take some control over the operations of my body (Foucault, 1997), fighting its natural shape by engaging with self-regulatory practices [taking duromine and thyroid medication even though my thyroid function was healthy, abuse of laxative medication and purging after meals]. Mandy also took some control back by practicing purging after meal times, as a means of maintaining a body weight which was accepted by coaches and team managers.

These practices were an act of conformation within the discourses of performance and perfection, and perhaps also "an ethic and aesthetic of selfmastery and self-transcendence, expertise, and power over others through the example of superior control" (Bordo, 1989, p. 23). For me, the descent of my weight on the scales gave me comfort and proof that I could exert control. I discovered admiration from my shrinking swimmer body, not as an aesthetic object but for the strength of my will and self-control in attempting to attain body perfection. Mandy saw purging as a practice that allowed her to eat while still 
maintaining the ideal swimmer body. It was a mechanism that she adopted and which she regarded as giving her back power and control. Mandy and myself were seduced by the rhetoric of performance and perfection and drew upon "acquired resources" (Evans, Rich \& Holroyd, 2004, p. 136) to help achieve swimmer body perfection. At the time, these acquired resources, signified success and a regain of self-control.

Jenny: I have found an answer.

Jenny: Everything will be ok now.

Mandy: This is going to fix everything. This will help me control my bodymy weight

Rich (2003) states:

As we have seen, within the discourse of healthism, thinness becomes the marker of the good student, self-controlled citizen, the subject who is conforming to the predetermined norms around the body. It is very much about the neo-liberal individual who is willing to work on themselves, demonstrate self-control. (cited in Rich \& Evans, 2007, p. 49)

If we apply Rich's (2003) thinking to the culture of sport and the discourse of performance and perfection, we can surmise that the swimmer body who meets expected body norms through appearance, through weight and skin fold measurements is seen to be a 'good athlete, self-controlled in their eating, an individual who has worked on themselves.' My internal dialogue exemplifies this as well as the voice of a senior female swimmer on the same representative team as Mandy.

Coach: “Jenny, you don't need to lose any more weight. You have done a good job."

My response (internal): I can lose more weight. I will show him! I have the power now. 
My response (internal): I feel accepted finally. I have proven that I can stop eating and lose weight.

Senior female swimmer (talking to Mandy): “After each meal, I just stick my fingers down my throat and vomit. It is a way that I can eat and still maintain my weight. I never get in trouble for my weight. Everyone thinks I am so controlled."

My shrinking body assisted in the defining of my swimmer self and subsequently social privileges occurred through the admiration of others in what I was achieving bodily. After eight years of trying, I finally heard those words. They were words that I had been waiting for, trying for so long to achieve. I believed, at that time that they were the last thing I needed to become an Olympic representative.

Coach: "Jenny, you don't need to lose any more weight. You have done a good job but that is enough!",

However, even though my swimmer body had 'slimmed to win' and met the approval of my coach, it was far from achieving Olympic representation. Below is my story of experience from this time.

I finish the warm up and feel as though I have swum nine kilometres. I am exhausted already. I commence the heart rate set with the rest of the squad. I have to do $20 \times 100$ 's of freestyle on the two minutes. I touch the wall after the first one. My coach yells:

Coach: "1.07."

I grab the heart rate monitor and put it onto my chest.

Coach: "186."

It is going to be a long morning. I have only swum $1 \times 100$ and my heart rate is already at its maximum. I am just not swimming the times that I was four months ago.

At least my weight loss is going well. 
(McMahon \& Dinan-Thompson, in press)

\section{Differential Power}

Within the context of the Australian swimming culture, power differentials in the coach-swimmer and/or the swimmer-manager relationship were experienced by all three swimmers as we became immersed in the discourses of performance and perfection. In so doing, we obligingly engaged in cultural practices that simultaneously aligned and normalized coaches,' managers' and athletes' behaviours within an inequitable power-relationship.

Carly, Mandy and myself did not question and subsequently consented to the philosophies, practices and pedagogies of our own personal coaches and the coaches and managers on representative teams. Our compliance was motivated by performance discourses, in terms of the results that our coaches had produced with other swimmers' performance. We willingly adopted the everyday routines of a swimmer (Giddens, 1998). For Mandy, her coach had major success at the pinnacle of the sport - the Olympic Games, producing numerous national representatives each year. Subsequently, she never questioned his philosophies, training regimes, because to her, he was the bearer of knowledge, the necessary key needed for her to achieve her goal of Olympic representation. Below, my words from 1999, reveal my surrendering of my body's voice - my power to my coach - also a producer of champions.

Here I sit on the side of the pool ... waiting ... ready ... for your master critique.

My body is yours ... make it win ... I look up to you ... following the rest ...

I will do what you want ... to be the best. I listen to HIM now not you ... one of my voices say. The other conveys ... this is not the way. An internal battle of the voices transpires ... momentarily ... before one says ... if you fail ... you will pay. 
Olympic representation I want ... he can give me ... Not you I say like past history.

The struggle continues ... momentarily ... Listen to me ... NO I say ... my coach is the only way! I ignore YOUR voice ... and ready myselffor his. He has produced champions in the past ... beside me they sit ... Listening to his voice ... IT is the key.

Similar to the work of Cushion and Jones (2006), Carly and I were obedient in regard to the practices of coaches and team managers, offering no resistance, obligingly consenting within the discourse/s of performance and perfection that persisted the greater cultural context of Australian swimming. In the main, Mandy displayed similar obedience, except for her story that featured in Act one, an extract of which is presented here:

Coaches: "If you are going to continue putting on weight, you are going to have to change your eating habits to keep your weight down."

I become infuriated. I know that I should not respond but I cannot help myself.

Mandy: "Look around you guys. Do you see many other swimmers here ranked number one in the world? Go for it, just do it!"

I can see that they are surprised by my response and they appear to back off.

Mandy's response, the assertiveness in her voice and the aggressive sarcasm (Willis, 1977) that she displayed, exemplified her questioning of the authority of the Australian team coaches that surrounded her. She subverted her language to which it would normally be expressed (Purdy et al., 2008). The situation portrayed an oppressive social environment (Willis, 1977) where Australian team coaches took power from Mandy at meal times by limiting her choice to just fruit and vegetables and controlling the place and space to which she allowed to consume the food.

When obligingly conforming to the practices described in the stories (Act one and Act two), our swimmer bodies were thus considered by coaches and team managers as biological objects to be unproblematically trained, manipulated and measured (Prain \& Hickey, 1995; Wright, 2000). We seemingly possessed an 
unquestioning, compliant and dependant athlete identity (Johns \& Johns, 2000), as we viewed the coach/s, team managers and physiologists as the bearer/s of knowledge of competitive performance. As such, we became the receivers of such knowledge (Jones et al., 2005). Subsequently, the power pervading the training environment and team situations that Carly, Mandy and I described, displayed various and constantly changing measures of compliance, cooperation and some resistance (Locke, 1985; Purdy et al., 2008). Our stories of experience, highlight the elusive everyday complexity of power and resistance which permeate sporting culture (Purdy et al., 2008; Strean, 1998) and furthermore, the power differential occurring in a coach-swimmer and manager-swimmer relationship. The experiences and relations described by us lead to an unquestioning, compliant and dependent athlete identity (Johns \& Johns, 2000), “where the coach is viewed as a knowledge giver and athletes as receivers who need that knowledge to better their performances" (Jones et al., 2005, p. 378). The practices remain neither questioned nor critically examined as "improved times and speed are often cited as 'proof' of its success" (Jones et al., 2005, p. 378). As Jones et al. (2005, p. 378) identified and our data has highlighted, such a "discourse takes little consideration of the personal circumstance and context of athletes."

Dependency and compliance arguably feature heavily in the stories presented in Act one. Mandy abided by coaches rules and limited her food intake, choice of food and where she sat to eat. Yet, this can also clearly be viewed as an act of denying Mandy social privilege, power and control at meal times. Similarly, as an 11 year old, Carly experienced an extreme form of disciplinary power and power differential when two male coaches woke her at $11 \mathrm{pm}$ at night and demanded that she run to burn the calories off from the ice-cream that she had consumed earlier that day. Running in the middle of the night, even when she complained of sore and blistery feet, resembled a punishment almost punitive in nature "submitting her [sic] to certain ends of domination" (Foucault, 1988, p. 18). For Mandy, Carly and myself complex differential power relationships within the coach-swimmer relationship pervaded every aspect of our swimmer lives and "the threat of punitive sanctions persuaded" us "to internalise the norms and values that prevailed within the social order" (Foucault, 1988, p. 18). Those differential relations are exemplified in our stories, inner dialogue and actions. They were portrayed and accepted as a necessity for optimal athletic performance. 
For us, they were seen as a tool to assist performance that was inscribed in our swimmer bodies, resonating with Foucault's (1977) account of 'normalization.' Like Foucault, Bordo (1997, p. 16) states that "the more a cultural practice is engaged in; by greater and diverse numbers of people, the more 'normal' it seems and the less likely we are to point the beam of evaluative or critical consciousness."

\section{0-30 years on: The effect}

Little is known about the long term impact that body pedagogies and power relations have on the body and the self. However, the data presented in Act three demonstrates the "durability" (Lee \& Macdonald, 2010, p. 203) of the practices occurring in discourses of perfection and performance. The perfection and performance practices continued to pervade the bodies of myself and the other two participants, some 10-30 years on in post swimming careers, perpetuated through our own self practices. Jackson (1990, p. 48) reveals how such experiences do have an undeniable 'effect' on the body and self.

Even though my body seems the most private and hidden part of me, I carry my life history in my body, almost like the way the age rings of a sawn tree trunk reveal the process through time. My personal history of social practices and relationships is physically embodied in the customary ways I hold my body, imagine its size and shape, and in its daily movements and interactions.

Like Jackson, the swimmers' bodies and the practices that they embodied during their adolescence have had an effect on our selves, shaping our identity and the practices that they make use of now as adults, some 10-30 years on. In an effort to make sense of the actors' stories as adults, Sparkes' (2004, p. 159) question focusing on embodiment is pertinent to this research "what do their [sic] memories reveal about the socialisation of [their] body?" Recent research on the body by Garrett (2004, p. 140) (see also Amour, 1999; Bordo, 1989, 1992; Frost, 2001; Grosz, 1995; Kirk, 1993) "suggest that bodies are both inscribed with and are vehicles of culture" revealing that "what we eat, how we dress and the way we 
act are not only inscribed and learned but serve as mechanisms of social control," Bordo (1993, p. 91) similarly contends that "through the regulation of time, space and movements of our daily lives, our bodies are trained, shaped and impressed" with the stamp of culture. Each of the actors embodied practices within the Australian swimming culture relating to the body. Learned behaviours are still evident in the way that they treat their bodies now as adults, suggesting that their bodies were impressed with the practices of the Australian swimming culture. For each of us, our exposure and adoption of practices and behaviours has had a sustained lived effect on our lives and our health and wellbeing. For both Mandy and Carly, 20 years on from their punishment for eating as adolescents, eating remains a "corporeal [bodily] sin" (Evans, Rich \& Holroyd, 2004, p. 126) that fills them with anxiety thus "dismantling it as a pleasurable experience" (Evans, Rich \& Holroyd, 2004, p. 126). Food still resembles an 'eating toxin' (adapted from Evans, Rich \& Holroyd, 2004), as they become overridden with guilt and toxic thoughts once the food is consumed and as they continue to engage in a “cult of slenderness" (Tinning, 1985; see also Evans, Rich \& Holroyd, 2004, p. 125). For Mandy, the "acquired resource" (Evans, Rich \& Holroyd, 2004, p. 136) of purging which she was introduced to in 1978 by another female swimmer is still being practised in an attempt to take back control at meal times and give her what she views as self-determination over her body. My argument with my husband was in retaliation to his gaze and surveillance over the food choices that I selected to feed our children. It was a reaction to the gaze and surveillance that I experienced as an adolescent in relation to my own eating (McMahon \& DinanThompson, 2008). The choices that I made for our children were for me, an act of regaining control at meal times as I rebel against what is viewed as 'external regulation' in my parenting. At each meal time I would experience an internal battle over what to feed them and usually, choose the food that was withheld from me as an adolescent. This battle has also pervaded my own eating for the last two years, with the choice of food high in sugar and high in fat representing a choice that I can now make and a degree of control that I was denied throughout my swimming career.

Collectively, the three adult bodies exemplify Bordo's (1989, p. 13) notion that the body is a "text of culture, an account, a rendering of cultural rules, a locus of social control." These stories reveal the marriage between exposure and effect 
as the three actors' bodies remain troublesome, representing the extent to which "the power has seeped into the very grain of our being, reaching right inside our bodies, permeating our gestures and how we live" (Sheridan, 1980, p. 217).

\section{Subjects of our own surveillance}

When applying Foucault (1977) to our adult bodies, Mandy, Carly and I have remained the subjects of our own surveillance for some 10-30 years after being immersed in the Australian swimming culture. As adolescents, we cast the gaze back onto ourselves as we became influenced by the normalizing gaze of coaches, team managers, physiologists, other swimmers and parents, a surveillance that classified and subsequently punished our swimmer bodies. As a consequence at this time, we engaged in practices in an attempt to conform to the ideal swimmer body.

Some 10-30 years on as we continue to cast the gaze back onto ourselves, managing our bodies by taking 'personal responsibility' (Johns \& Johns, 2000) for them in bodily practices that continue to permeate our everyday life. Mandy and Carly continue to practice excessive self surveillance by weighing themselves countless times every day. The practice of daily weigh-ins was introduced to all three of us in our adolescents, implemented by coaches and team managers to ensure our weight was maintained. This practice was normalized, and now subsequently as adult women continues to still be normalized and engaged with on a daily basis. However as Carly revealed, it is a daily reminder of how her body failed to conform as an adolescent and continues to fail now. Below the words of Carly as an adult woman:

Carly: I look down at my weight. I am 0.25 of a kilogram heavier than what I was two hours ago. Heaviness drapes over my shoulders. I feel flat, deflated. I hate myself. I am disgusting.

For Mandy, being weighed in hospital before an operation without being told her result exacerbated her self-consciousness in regard to her weight and body measurement. Below the inner dialogue and words of Mandy and the context to which this occurred: 
Mandy: I wonder what she means by that comment. Does she mean that I have lost weight or put on weight? My mind starts racing as panic starts to overcome me. I need to know how much I weigh. I want to know now. It becomes too much. I haven't weighed myself since yesterday and I am concerned at the nurse's response.

Mandy: "Can you tell me my weight please?"

The nurse then puts the blood pressure cuff on and it seems like she has not heard me.

Mandy: "Tell me my weight please!"

This time my voice is raised.

Unlike Carly and Mandy, as an adult, I have challenged the practice of weighing myself daily. I now avoid scales all together. I have realised the emotional torment associated with the knowledge of the number, the emotional highs and lows that become attached to the natural fluctuations in weight. For me standing on the scales is a reminder of my experiences as an adolescent, the heart ache that was associated with trying to achieve the ideal swimmer body, the emotional and physical pain associated with the punishment that I received for not conforming to what was expected. I now avoid scales as it is my way of taking back the control that I did not have as a 16 year old. Yet while I endeavour to avoid a situation of scales and weight dictating my feelings and behaviours, I still manage my adult body taking 'personal responsibility' (Johns \& Johns, 2000) for its shape by exercising on a daily basis, privately striving for that swimmer body and self punishing myself if I miss a session. The routine, discipline and 'personal responsibility' as described by Johns and Johns (2000), is something that I progressively returned to. For eight years, in what I thought was an act of regaining power and control over my body and eating, I consciously chose to eat whatever I wanted and did not engage in any physical activity at all.

It was upon conclusion of this research where both Mandy and Carly realised that there was an apparent connection between the exposure and effect of body practices, admitting that since adolescence they have continued to practice self surveillance over their bodies, and in so doing are highly critical as their 
bodies seemingly are bound to their own perceptions of whether it is slim enough. Thus, the discourse of perfection continues to pervade their bodies as adult women in regard to their own perceptions of their self and their own practices that were normalized in adolescence and subsequently have become "regimes of truth" (Jones et al., 2005, p. 386). Hall (1996, p. 6) states, "identities are created through the successful chaining of the subject to the discourse," thus the discourse of perfection which Carly and Mandy are still bound to continues to dictate how they think of their bodily selves (Jones et al., 2005). I asked both Mandy and Carly whether they engage with discourses of performance and perfection in relation to others, placing the same expectations on others [family members, athletes, students] as themselves. They both replied that they could not subject others to the same criticism as they endured as adolescents in relation to the perfection of their body and also performance. More specifically, it was revealed within the discourse of performance that Mandy challenged the body pedagogies that she was exposed to as an adolescent. When her own swimmer failed to reach her performance expectations, she reacted in a contrast to that of her own coach. Mandy's reflexivity and focus on the 'other' [Katie] was imperative in the challenging of body pedagogies relating to performance, revealing the importance of agency and knowledge. Ironically, even though the 'other' was a significant factor in challenging body pedagogies relating to performance, they are still prepared to engage in these pedagogies themselves.

\section{Fractured bodies}

In the introduction to this thesis, I highlighted how self had been italicised throughout the research as it denoted the importance of the body, being and self as one static entity. And how for me, the self encompassed the union of all elements including; emotions, spirituality, thoughts, sensations, experiences, conversations which constitutes the individuality and identity of each person, as a soul, underlying all subjective experience. Trinh (1999) echoes similar sentiments to my interpretation of the self and body revealing that the body is not a possession of higher mind, to be manipulated and controlled to serve the brain; the body and the person-self are one. However, the data showed the ways in which our three swimmer bodies were fractured as we referred to them in third person, blaming 
them for their failure to conform to the accepted ideal. What has become apparent some 10-30 years on for me, even after the discourse of performance and perfection has been withdrawn is that a fractured body/self persists. This has been exemplified through my writing as I have seemingly referred to not only my swimmer body but also the bodies of Carly and Mandy in third person in and through the critique. Ellingson (2006) reveals how language conventions make it difficult to include the body as the self. However, in the writing of the body in this research, my sub consciousness "of my body in how I [continue to] experience it and perceive it" (Ellingson, 2006, p. 303) has filtered through into my writing genre. In so doing, as the researcher of this production, I have inherently written my body and the body of the other two participants in third person throughout the critique thus revealing my detached prose and subsequent persistent possession of a fractured body/self revealing how my writing is a "deeply embodied process" (Ellingson, 2006, p. 304). As Ellingson (2006) described, my embodied experience demystifies the process of my research and writing because it is "clear that my body could not be separated from and dominated by my mind's more logical perspective" (p. 304).

\section{Conclusion}

The lived experiences of the three swimmers in this research may not represent nor resonate with the experiences of all competitive swimmers within the Australia swimming culture or internationally. They are, however, experiences that articulate a sporting culture built on performance and perfection which indicatively point to individuals holding positions of power and authority in the Australian swimming culture having neglected to adequately reflect or "point the beam of evaluative or critical consciousness" (Bordo, 1997, p. 16) on the effects of bodily practices, ideas and power. Drawing on the work of Foucault (1977) and others to probe and understand the experiences of the three swimmers, this thesis has attempted to provide an insight into the explicit exposure of cultural bodily practices and potential effects of a sporting culture. The intention has been to enhance understanding of body pedagogies within the cultural context by articulating particular practices and memes relating to the swimmer body. The stories presented have revealed that some 10-30 years on from their adolescent 
experiences and participation, all three swimmers have embodied a fractured swimmer identity that continues to pervade at meal times and in the way that they treat their bodies and selves. A link between the exposure and effect of the body and a 'connection of the dots' (Klein, 2000) is inherent in the move from Act one to Act two and Act three. It is impossible to ignore the interplay between the body, power and knowledge and the effects that culture have on the body and selves both in the short term and long term. Further examination of the effects of "a cultural approach to the body by looking at the obsessive body practices of contemporary culture" (Bordo, 1993, p. 35) and in particular the micro-culture of Australian swimming, is undoubtedly needed. The effects of experiences of the three swimmers are vividly depicted in the stories presented and from the athlete perspective, are still lived out. My ruminations on the stories included in this chapter are embodied, not always resembling that of a "traditional pathological perspective," (Rich et al., 2004, p. 173), but resonate ongoing corporeal and emotional tensions that present challenges in everyday life and also in the contexts of research and academic writing. In writing this thesis I have endeavoured to engage with those challenges. 
CHAPTER SIX 


\section{Conclusion}

In this final chapter, I draw upon "the personal and social significance" of this study (Clandinin \& Connelly, 2000, p. 161) to reveal my learning in relation to the research questions and to critically reflect on the research process by identifying issues that this research has brought to the fore. In this research, I suggest that there can be some link drawn between exposure and effect of body pedagogies. Body pedagogies occurred in and through the discourse of perfection and performance within the Australian swimming culture. Given the longitudinal dimension to this research, the durability of body pedagogies, particularly pedagogies occurring within the discourse of perfection, was evident in the stories revealed by the participants as adult women. In so doing, the data pointed to a continuation of body pedagogies and ongoing acceptance and reproduction of the discourse of perfection for each of the three participants. However within the discourse of performance and the body pedagogies occurring within it, some agency was nevertheless demonstrated by each of us.

As the producer of the acts and author of this thesis, I have grappled with challenges of representation, particularly to ensure that the stories were told in a way that retained their authenticity. In so doing, this thesis presented three swimmers' stories which were not intended to be representative in any way of all swimmers, but rather acknowledged as centring on individual experiences that raise important issues for all involved in the sport. I intended that in engaging with the participants' storied lives, the reader would become integrally involved in the research, making meaning through their individual reading,' thought and discussion (Gray, Ivonoffski \& Sinding, 2001). Attempting to articulate any definitive conclusions at this point in the research process is arguably problematic. It potentially interrupts the meaning making process for the reader. Thus, this concluding chapter articulates my meaning making and acquisition of knowledge during the research, with comment made in regard to recommendations for further research. Readers may well come to different conclusions from their own acquired knowledge and journey through the thesis.

My interrogation into the culture of Australian swimming was directed by a number of research questions. The overarching question of this thesis was: 
- What are the effects of the body pedagogies of a culture?

Four supporting questions were:

- What body pedagogies within the discourse of perfection were each of the three swimmers exposed to as adolescents during their immersion in the Australian swimming culture?

- What body pedagogies within the discourse of performance were each of the three swimmers exposed to as adolescents during their immersion in the Australian swimming culture?

- What body pedagogies do the three participants still engage with some 10-30 years after being immersed in the Australian swimming culture?

- Can a link be made between the exposure and effect of body pedagogies occurring within a culture?

\section{Research significance: Revealing my learning - Act One and Act Two}

For me, the data revealed that body pedagogies occurred in and through the discourse of perfection within the Australian swimming culture. The ideology of 'slim to win' pervaded the discourse of perfection based on amelioration [making or becoming better] which "define[d] the significance, value and potential of the [swimmer] body" (Evans et al., 2008, p. 17). The normalisation of particular body pedagogies within the Australian swimming culture was revealed. Body pedagogies occurred within the discourse of perfection via social interactions, practices and methods. Our three swimmer bodies were managed, regulated, measured and compared for the sake of [bodily] perfection with the intention of enhancing competitive performance.

In the elite and amateur sub contexts of Australian Swimming culture particular body pedagogies occurred because of a characterisation of "preoccupation with conformity, management and control over the [swimmer] body" (Garrett, 2004, p. 143). Daily and weekly weigh-ins were implemented on 
Australian swimming teams as well as in the amateur context. Monitoring of the swimmers' diet through surveillance and skin-folds measurements exemplified these body pedagogies and reinforced the discourse of perfection based on "values packaged in a scientific wrapping" (Vertinsky, 1985, p. 73). In this sense, science [ethnophysiology] defined body pedagogies, power relations and communication as they were the apparatus of control over the swimmer body. The idea of the perfect swimmer body was not a fixed entity. Rather, it was something that was progressively developed and legitimated through the emphasis on 'slim to win' body pedagogies.

The discourse of performance body pedagogies was shown to be underpinned by the ideologies of technocracy and meritocracy. Meritocracy fuelled technocentric ideologies as our three 'swimmer bodies' were "viewed as an instrument and object for manipulation" (Bain, 1990, p. 29), a commodity in order to achieve competitive success. The body pedagogies that occurred did so through ethnophysiological tests such as lactate tests and heart rate tests. These were implemented by coaches to ameliorate swimmer bodies, so that our fitness level and speed would be enhanced. When swimmers did not meet the expectations of their coach in training, they were punished with more mileage in the pool and/or other training (such as running). Socially, in the training environment, competitive environment and also home environment, dialogue and interactions were centred on competitive performance, specifically in regard to being selected on national teams and winning medals. This was apparent in both the amateur and elite contexts. Achievement of competitive performance was rewarded with social privilege while failure to achieve competitive performance engendered social isolation. Subsequently, it is in my view that Australian swimming was a culture where swimmers had "lost their claim to respect except in terms of performance" (Ball, 2004, p. 4). Within the discourse of performance, the complexity of body pedagogies was evident as the various links between pedagogy, power, control, embodiment and swimmer identity were revealed. Our own knowledge and practices altered as we embodied body pedagogies relating to performance and in so doing, normalised them. 


\section{Research significance: Revealing my learning - Act Three}

Given the longitudinal dimension to this research, the durability of body pedagogies, particularly within the discourse of perfection, was evident in the stories revealed in Act three. For each of the three participants, some 10-30 years after being immersed in the Australian swimming culture, I suggest that the data pointed to a continuation of body pedagogies and ongoing acceptance and reproduction of the discourse of perfection. Specifically, the pedagogic practices engaged with as adult women included; disordered eating, constant monitoring of weight, self surveillance, and self worth being linked to weight. The body pedagogies, including social ideas, values and practices presented by the Australian Swimming culture became part of our embodied selves, which in turn affects the decisions we make as adult women in regard to our bodies. Although two of us maintained critical distance from the Australian Swimming culture for a period of 10 and 30 years respectively, all three of us have continued engagement with body pedagogies that align with our adolescent swimmer experiences. As discussed in chapter five, the data indicated the "durability" (Lee \& Macdonald, 2010) of the body pedagogies we experienced and the discourses that they embodied and legitimated. Practices such as self-surveillance over our own bodies were normalised as adolescents. As adult women, we continue to normalise the practice.

Although we were undoubtedly in a situation of unequal power-relations, we nevertheless retained some agency in relation to the adoption of particular discourses and associated pedagogical practices. We had some scope to challenge the dominant discourses, and widely accepted normalised pedagogical practices that we were exposed to as adolescents. As coaches and/or teachers, each of us has at least to some extent strived to base our interactions and practices on discourses other than performance. At times we have all fore grounded discourses of wellbeing, placing our attention on this outcome rather than that of performance. Our reflexivity and focus on the 'other' was imperative in the challenging of body pedagogies relating to performance, revealing the importance of agency and knowledge. While I suggest that there can be some link drawn between exposure and effect of body pedagogies, I also now recognise the importance of agency in the challenging of body pedagogies and culture. 
Shilling's (2004) idea of how social meaning relating to the body can be disrupted through self reflection and knowledge becomes pertinent as it occurred in all three of our cases relating to the discourse of performance. The effects of the body pedagogies of the Australian swimming culture were revealed by all three of us and so to was their continuation, ongoing acceptance and reproduction particularly within the discourse of perfection. Although, as adult women within the discourse of performance, some agency was nevertheless demonstrated by each of us.

\section{Recommendations for future research}

This research has articulated body pedagogies occurring in and through the Australia swimming culture and has provided some vivid insights into their subsequent and longstanding effect. Understanding the broader context of body pedagogies and more specifically, the ways in which values and culture frame body pedagogies needs further investigation. Pedagogy is the active realisation of knowledge (Esland, 1971). As such, my gaze arguably needs to now shift from the effects of pedagogy, to the connection between acquisition and practice of pedagogy. Therefore, my recommendations for further study relate to coach education programmes and the processes of pedagogy acquisition. Specifically, there is a case for investigating whether the storied representations of elite and amateur swimmers (similar to those described in this research) have a role to play in challenging existing body pedagogies. As the current Australian swimming coach of the year, Michael Bohl (2010), illustrates, ideologies of 'slim to win' and the associated practices (i.e., weigh-ins/skin fold tests) within the discourse of perfection still pervade Australian swimming culture today.

Douglas and Careless (2008, p. 35) reveal the potential of storied representations saying, "stories stimulate new ways of seeing the world." Therefore, I would like to see future research that explores body pedagogies and the practices and social interactions associated with it employing narrative methods, allowing coaches participating in the research an opportunity to share their responses and feelings and also enabling the reader to vicariously share in their responses. Notably, Douglas and Careless (2008) conducted a study with 
professional golf coaches with promising findings. Throughout a series of continuing professional development seminars for professional golf coaches, the lived experiences of elite professional golfers were presented in the form of stories and poems. Following these presentations, they obtained written responses to the stories from 53 experienced coaches who attended the seminars. Analysis of this data revealed three ways in which the coaches responded to the stories. The first included questioning in the form of the action of challenging, seeking further information and reflective practice. The second response included, 'summarising' through synopsis and the act of active listening that led them to draw their own conclusions. Finally, the final way the coaches responded to the stories included 'incorporating' [incorporation of the athletes' story into their own experience as well as displaying emotional reactions to stories such as empathy]. Thus, their data revealed the potential for storied forms of representation "to enhance professional development through stimulating reflective practice and increasing understanding of holistic, person-centred approaches to coaching athletes in highperformance sport” (Douglas \& Careless, 2008, p. 33). Further, the recorded responses in their research revealed how storied representations stimulated the coaches to critically reflect and think about the issues of the athletes. Their findings suggest "that stories can provide a catalyst for coaches to explore their own subjective, moral and ethical beliefs in a supportive environment" (Douglas \& Careless, 2008, p. 46).

Prospectively, therefore, the knowledge gained from this type of research with swimming coaches could enable a greater awareness of body pedagogies, by pointing a "beam of evaluative or critical consciousness" (Bordo, 1997, p. 16) upon longstanding accepted practices. The underlying intention of such research would be pedagogical, structural and cultural exploration in swimming coaching contexts and cultures.

\section{Ruminations of a journey}

At the beginning of this research journey, I referred to my research project as a journey. In the concluding stages of this research my sociological understanding has developed. I now realise that my research journey has involved (at least) two 
journeys of discovery. While this research centred on excavating the exposure and effect of body pedagogies of myself and two other participants, the research process has been a parallel journey of learning and discovery. In this section, I reveal my ruminations of the continuing narrative of these two journeys.

Taking on the dual role of primary researcher and participant, my initial challenge was to effectively and appropriately 'juggle' my multiple selves in and through the research journey as I took on varying roles, as academic, participant and former teammate [when interviewing the other two participants]. During the course of the research journey, adaptations to the various roles became easier, such that I have come to think of myself as a 'chameleon' (Somekh, 2006) seamlessly adjusting to the particular role/discourses in which I have been immersed [academia, Australian swimming culture, researcher and coparticipant].

Prior to commencing this doctoral research, I was aware that my feelings towards my body and in particular my experiences in the Australian swimming culture were unresolved. I grappled with this initially, concerned that my state of being could seriously interfere with my learning processes and affect the outcome of the research, including the way that I wrote and presented the findings. This concern was compounded by the fact that the topic of this research was inevitably something that I felt strongly about and continued to present me with problems as my body and self remained fractured. Initially I attempted to resolve my mixed feelings through extensive scholarly reading, largely to no avail. My being still remained somewhat illogical in regard to my adolescent experiences. However, like Richardson (1994), the process of writing was a 'method of inquiry,' something that assisted me in coming to terms with my adolescent experiences within the Australian swimming culture. Richardson states:

Although we usually think about writing as a mode of 'telling' about the social world, writing is not just a mopping-up activity at the end of a research project. Writing is also a way of 'knowing' - a method of discovery and analysis. By writing in different ways, we discover new aspects of our topic and our relationship to it. Form and content are inseparable. (Richardson, 1994, p. 516) 
My writing became a method of knowing and a method of self discovery, a tool that challenged my preconceptions. It was a way of coping with my feelings, separating what I regarded as 'the logical' from the seemingly 'illogical.' Notably, I am now acutely aware that writing is a progressive process. As Frank (2000) says:

those who accept an invitation into the storytelling relation open themselves to seeing (and feeling and hearing) life differently than they normally do. Listening is not so much a willing suspension of disbelief as a willing acceptance of different beliefs and of lives in which these beliefs make sense ... Those who have accepted the invitation to the story may not choose to remain in the world of the story, but if the story works, then life in their worlds will seem different after they return there. (p. 361)

Early on in my writing, I found that I needed to assemble an initial draft where I could reveal all of my heartfelt feelings; including criticisms of what seemed to me to be poor treatment of adolescent swimmers, revelations of what my own and fellow participants' data might imply, recognition of issues that may be raised to counter particular ideas and arguments, and admissions of where the gaps in my arguments were. This writing was never intended for others to view, but rather, served to set free my inner most emotions, enabling my academic self to be less clouded.

As the producer and director of this research production, I also faced ethical responsibilities in regard to what I chose to disclose in some of my personal stories. I was aware that the need to "write persuasively so that the reader experiences being there" (Cresswell, 1998, p. 21) raised dilemmas in retaining the sensitivity to interests of others implicated in my stories. Several of my initial autoethnographic accounts had distinctive features and included private conversations with my family, whose consent I had not sought. Consequently, I chose to remove some details due to the private and intimate nature of the storied accounts and the ethical issues that can be associated with revealing information. In this process, I sought to avoid sacrificing the intent of the stories. 
As I approach the completion of this journey I am able to cast the beam of consciousness (Bordo, 1997) back over my own personal learning journey and realise how my own knowing has progressed significantly, as has the relationship with my body since the commencement of this journey. My personal growth throughout this journey has been deeply empowering. While I see the importance of knowledge and agency in challenging body pedagogies and dominant forms of power and control, I also acknowledge how my new subjectivity is able to shield and deconstruct future dominant ideologies in regard to the body, power and control. This newfound knowledge and confidence in turns empowers me to continue this research journey, with the intention of influencing others through future research. For me, this journey will continue with the hope of further emancipation such that my body and self will become one again.

\section{Closing thoughts in relation to the research questions}

It was my intention at the conception of this thesis that the findings, specifically the voices of experience of myself and the other two swimmers would bring about questioning, some acquisition of knowledge into the sporting culture of Australian swimming. The Australian swimming culture currently produces a swimmer body formed through 'pedagogy,' more specifically body pedagogies, 'traditions' [as revealed over a 30 year period], 'practices' and 'coach education.' Thus, it was my intention that through the revelations of this research that the pedagogy, methods and practice involved in forming this swimmer body would be brought to the fore. In so doing, the recipe of how this swimmer body is constructed would be questioned with consideration made into wellbeing. In closing, I find Foucault's (1997, pp. 295-296) thoughts particularly pertinent:

If one is to challenge the domination of particular truths, a particular truth regime, then they must do so by playing a certain game of truth, by showing its consequences, by pointing out that there are other reasonable options, by teaching people what they don't know about their own situation, their working conditions and their exploitation. 
I can offer no conclusive interpretation of the findings, my experiences or the experiences of Mandy and Carly. I see and feel no conclusions "but rather ambiguities, contradictions and openings" (Smith, 1999, p. 275). I anticipate further exploration in future research, searching for answers with the hope of not only personal closure but also, an assurance that the body pedagogies that we were exposed to will be critically examined and further challenged by those involved in the Australian swimming and sporting culture. 


\section{REFERENCES}


Andrews, D. (2000). Posting up: French post-structuralism and the critical analysis of contemporary sporting cultures. In J. Coakley \& E. Dunning (Eds.), Handbook of sport studies (pp. 106-138). London: Sage.

Amour, K. (1999). The case for a body-focus in education and physical education. Sport, Education and Society, 4, 5-15.

Arthurs, J., \& Grimshaw, J. (1999). Introduction. In J. Arthurs \& J. Grimshaw (Eds.), Women's bodies: Discipline and transgression (pp. 1-16). London: Cassell.

Austin, J. (2005). Culture and identity. Australia: Pearson Education.

Aycock, A. (1992). The confession of the flesh: Disciplinary gaze in casual bodybuilding. Play and Culture, 5, 338-357.

Bain, L. (1990). A critical analysis of the hidden curriculum in physical education. In D. Kirk \& R. Tinning (Eds.), Physical education, curriculum and culture: Critical issues in the contemporary crisis (pp. 23-41). Hampshire, UK: Falmer Press.

Ball, S. (2004). Performativities and fabrications in the education economy: Toward the performative society. In S. Ball (Ed.), The RoutledgeFalmer reader in sociology of education (pp. 143-155). London:

RoutledgeFalmer.

Bartky, S. (1988). Foucault, femininity and the modernization of patriarchal power. In I. Diamond \& L. Quinby (Eds.), Feminism and Foucault: Rejections on resistance (pp. 61-86). Boston: Northeastern University Press.

Beckett, L. (2004). Health, the body, and identity work in health and physical education. Sport, Education and Society, 9(2), 171-175.

Bernstein, B. (1975). Class, codes and control. Vol. 3. Towards a theory of educational transmission. London: Routledge.

Bernstein, B. (1996). Pedagogy, symbolic control and identity. London: Taylor \& Francis.

Bernstein, B. (2000). Pedagogy, symbolic control and identity: Theory, research, critique. London: Rowman \& Littlefield.

Boog, B. (2003). The emancipatory character of action research: Its history and the present state of the art. Journal of Community and Applied Social Psychology, 13, 426-438.

Bordo, S. (1989). The body and the reproduction of femininity: A feminist appropriation of Foucault. In A. Jagger \& S. Bordo (Eds.), Gender, body, knowledge: Feminist reconstructions of being and knowing (pp. 13-33). New Brunswick: Rutgers University Press. 
Bordo, S. (1992). Postmodern subjects, postmodern bodies. Feminist Studies, 18, 159-176.

Bordo, S. (1993). The body and the reproduction of femininity. In S. Bordo (Ed.), Unbearable weight: Feminism, western culture, and the body (pp. 90110). Los Angeles: University of California Press.

Bordo, S. (1996). Anorexia nervosa: Psychopathology as the crystallization of culture. In A. Garry \& M. Pearsall (Eds.), Women, knowledge and reality: Explorations in feminist philosophy (pp. 388-418). Routledge: New York.

Bordo, S. (1997). Twilight zones: The hidden life of cultural images from Plato to $O$. J. Berkley: University of California Press.

Bourdieu, P. (1977). Outline of a theory of practice. Cambridge: Cambridge University Press.

Bourdieu, P. (1979). Distinction: A social critique of the judgement of taste. London: Routledge.

Bourdieu, P. (1984). Distinction: A social critique of the judgement of taste $\left(^{\text {nd }}\right.$ ed.). London: Routledge.

Bourdieu, P. (1986). The forms of capital. In J. Richardson (Ed.), Handbook of theory and research for the sociology of education (pp. 241-258). New York: Greenwood Press.

Bourdieu, P. (1988). Homo academicus. Stanford, CA: Stanford University Press.

Bourdieu, P. (1990). The logic of practice. Cambridge: Polity Press.

Bourdieu, P. (1991). Language and symbolic power. Cambridge: Polity Press.

Brownwell, K. (1991). Dieting and the search for the perfect body: Where physiology and culture collide. Behaviour Therapy, 22, 1-12.

Bruner, E. (1986). Ethnography as narrative. In V. Turner \& E. Bruner (Eds.), The anthropology of experience (pp. 139-158). Urbana, IL: University of Illinois Press.

Bruner, J. (1987). Life as narrative. Social Research, 54, 11-32.

Butler, J. (1990). Gender trouble, subversion and the foundation of identity. New York: Routledge.

Butler, J. (1994). Gender as performance. Radical Philosophy, 67, 32-39.

Carlisle-Duncan, M. (1994). The politics of women's body images and practices: Foucault, the panopticon, and shape magazine. Journal of Sport and Social Issues, 18(1), 48-65.

Chapman, G. (1997). Making weight: Lightweight rowing, technologies of power, and technologies of the body. Journal of Sport and Social Issues, 17(2), 77-97. 
Clandinin, D. J., \& Connelly, F. M. (1991). Teacher as curriculum maker. In. P. Jackson (Ed.), Handbook of research on curriculum (pp. 363-401). New York: Macmillan.

Clandinin, D. J., \& Connelly, F. M. (2000). Narrative inquiry: Experience and story in qualitative research. San Francisco: Jossey-Bass.

Cliff, K., \& Wright, J. (2010). Confusing and contradictory: Considering obesity discourse and eating disorders as they shape body pedagogies in HPE. Sport, Education and Society, 15(2), 221-233.

Cole, C. (1993). Resisting the canon: Feminist cultural studies, sport and technologies of the body. Journal of Sport and Social Issues, 17(2), 77-97.

Connelly, F. M., \& Clandinin, D. J. (1990). Stories of experience and narrative inquiry. Educational Researcher, 19(5), 2-14.

Cortazzi, M. (1993). Narrative analysis. London: Falmer Press.

Creswell, J. (1998). Qualitative inquiry and research design: Choosing among five traditions. Thousand Oaks, CA: Sage.

Crossley, N. (1996). Body-subject/body-power: Agency, inscription and control in Foucault and Merleau-Ponty. Body and Society, 2(2), pp. 99-116.

Crossley, N. (2001). The social body: Habit, identity and desire. Thousand Oaks, CA: Sage.

Crossley, N. (2004). The circuit trainer's habitus. Body and Society, 10(1), 37-69.

Crossley, N. (2006a). The networked body and the question of reflexivity. In D. Waskul \& P. Vannini (Eds.), Body/Embodiment (pp. 21-34). Aldershot: Gower.

Crossley, N. (2006b). In the gym. Body and Society, 12(3), 23-50.

Crossley, N. (2007). Researching embodiment by way of body techniques. In C. Shilling (Ed.), Embodying sociology (pp. 80-94). Oxford, UK: Blackwell.

Cushion, C., \& Jones, R. (2006). Power, discourse and symbolic violence in professional youth soccer: The case of Albion F. C. Sociology of Sport Journal, 23(2), 142-161.

Danaher, G., Schirato, T., \& Webb, J. (2000). Understanding Foucault. London: Sage.

Davis, Z. (2004). The debt to pleasure: The subject and knowledge in pedagogic discourse. In J. Muller, B. Davies \& A. Morais (Eds.), Reading Bernstein, research Bernstein (pp. 44-57). London: RoutledgeFalmer.

Dawkins, R. (1989). The selfish gene (2 ${ }^{\text {nd }}$ ed.). Oxford, UK: Oxford University Press. 
Denison, J. (2007). Social theory for coaches: A Foucauldian reading of one athlete's poor performance. International Journal of Sports Science and Coaching, 2(4), 369-383.

Denzin, N. K. (1997). Interpretive ethnography: Ethnographic practices for the $21^{\text {st }}$ century. Thousand Oaks: Sage.

Douglas, K., \& Careless, D. (2008). Using stories in coach education. International Journal of Sports Science and Coaching, 3(1), 33-49.

Dowling-Naess, F. (2001). Narratives about young men and masculinities in organised sport in Norway. Sport, Education and Society, 6, 125-142.

Drummer, G., Rosen, L., Heusner, W., Roberts, J., \& Counsilman, J. (1987). Pathogenic weight-control behaviours of young competitive swimmers. The Physician and Sports Medicine, 15, 75-84.

Duncan, M. (1994). The politics of women's body images and practices: Foucault, the panopticon and shape magazine. Journal of Sport and Social Issues, $18,48-65$.

Eisner, E. (1985). The educational imagination. New York: Macmillan.

Ellingson, L. (2005). Communicating in the clinic: Negotiating frontstage and backstage teamwork. Cresskill, NJ: Hampton.

Ellingson, L. (2006). Embodied knowledge: Writing researchers' bodies into qualitative health research. Qualitative Health Research, 16(2), 298-310.

Ellis, C. (1995). Final negotiations. Philadelphia: Temple University Press.

Ellis, C., \& Flaherty, M. (1992). Investigating subjectivity: Research on lived experience. London: Sage.

Ellis, C., \& Bochner, A. (2000). Autoethnography, personal narrative, reflexivity: Researcher as subject. In N. K. Denzin \& Y. S. Lincoln (Eds.), Handbook of qualitative research (pp. 733-768). London: Sage.

Esland, G. (1971). Teaching and learning as the organisation of knowledge. In M. Young (Ed.), Knowledge and control: New directions for the sociology of education (pp. 70-115). London: Macmillan.

Evans, J. (2004). Making a difference? Education and 'ability' in physical education. European Physical Education Review, 10(1), 95-108.

Evans, J., Rich, E., \& Holroyd, R. (2004). Disordered eating and disordered schooling: What schools do to middle class girls. British Journal of Sociology of Education, 25(2), 123-142.

Evans, J., Davies, B., \& Wright, J. (2004). Body knowledge and control: Studies in the sociology of physical education and health. Oxon, UK: Routledge.

Evans, J., Rich, E., Davies, B., \& Allwood, R. (2008). Education, disordered eating and obesity discourse: Fat fabrications. London: Routledge. 
Featherstone, M. (1991). The body in consumer culture. In M. Featherstone, M. Hepworth \& B. Turner (Eds.), The body: Social process and cultural theory (pp. 170-196). London: Sage.

Fetters, J. (1978). Sport, myth and the courage of self-creation. Quest, 3, 36-45.

Fleming, D. (Executive Producer). (2005). Meeting Miss Menzies [Television series episode]. Australian story. Retrieved August 8, 2008, from http://www.abc.net.au/austory/content/2005/s1303488.htm

Fontana, A., \& Frey, J. (2000). Interviewing: The art and science. In N. K. Denzin \& Y. S. Lincoln (Eds.), Collecting and interpreting qualitative materials (pp. 47-78). Newbury Park, CA: Sage.

Forster, C. (Producer). (2010). Woman on a mission [Television series episode]. Australian story. Retrieved May 5, 2010, from http://www.abc.net.au/austory/specials/womanmission/default.htm

Foucault, M. (1972). The archaeology of knowledge and discourse on language. New York: Pantheon Books.

Foucault, M. (1974). The order of things: An archaeology of the human sciences. New York: Vintage/Random House.

Foucault, M. (1975). The birth of the clinic: An archaeology of medical perception. New York: Vintage.

Foucault, M. (1977). Discipline and punish: The birth of the prison. London: Allen Lane.

Foucault, M. (1978). The history of sexuality: An introduction. New York: Pantheon.

Foucault, M. (1979). Discipline and punish: The birth of the prison. New York: Vintage Books. (Original work published, 1977).

Foucault, M. (1980a). Body power. In C. Gordon (Ed.), Power/knowledge: Selected interviews and other writings, 1972-1977. Brighton: Harvester.

Foucault, M. (1980b). The eye of power. In C. Gordon (Ed.), Power/knowledge: Selected interviews and other writings, 1972-1977. Harlow: Harvester.

Foucault, M. (1984). The subject and power. In B. Wallis (Ed.), Art after modernism: Rethinking representation (pp. 417-432). New York: Godine.

Foucault, M. (1988). Technologies of the self. In L. Martin, H. Gutman \& P. Hutton (Eds.), Technologies of the self: A seminar with Michel Foucault (pp. 16-49). Amherst, MA: University of Massachusetts Press.

Foucault, M. (1991). The Foucault reader. London: Penguin.

Foucault, M. (1996). Foucault live: Interviews, 1961-84. London: Semiotext(e). 
Foucault, M. (1997). Technologies of the self. In P. Rabinow (Ed.), Ethics: subjectivity and truth. Essential works of Foucault 1954-1984 (Vol. 1, pp. 223-252). London: Penguin.

Frank, A. (2000). Standpoint of storyteller. Qualitative Health Research, 10(3), 354-365.

Frost, L. (2001). Young women and the body. Basingstoke, UK: Palgrave.

Garrett, R. (2004). Gendered bodies and physical identities. In J. Evans, B. Davies \& J. Wright (Eds.), Body knowledge and control: Studies in the sociology of physical education and health (pp. 140-155). London: Routledge.

Geertz, C. (1991). Works and lives: The anthropologist as author. Stanford, CA: Stanford University Press.

Germov, J., \& Williams, L. (1999). Dieting women: Self-surveillance and the body panopticon. In J. Sobal \& D. Maurer (Eds.), Weighty issues: Fatness and thinness as social problems (pp. 117-132). New York: Walter de Gruyter.

Giroux, H. (1994). Towards a pedagogy of critical thinking. In K. Walters (Ed.), Re-thinking reason: New perspectives in critical thinking (pp. 200-201). Albany, NY: State University of New York Press.

Giddens, A. (1998). The third way: The renewal of social democracy. Cambridge, MA: Polity Press.

Gray, R., Ivonoffski, V., \& Sinding, C. (2001). Making a mess and spreading it around: Articulation of an approach to research-based theatre. In A. Bochner \& C. Ellis (Eds.), Ethnographically speaking: Autoethnography, literature and aesthetics (pp. 57-75). Walnut Creek, CA: Altamira Press.

Griffin, J., \& Harris, M. (1996). Coaches' attitudes, knowledge, experiences and recommendations regarding weight control. The Sport Psychologist, 10, 180-194.

Grosz, E. (1995). Space, time and perversion. London: Routledge.

Gubrium, J., \& Holstein, J. (1999). At the border of narrative and ethnography. Journal of Contemporary Ethnography, 28(5), 561-573.

Halas, J., \& Hanson, L. (2001). Pathologizing Billy: Enabling and constraining the body of the condemned. Sociology of Sport Journal, 18, 115-126.

Hall, A. (1996). Feminism and sporting bodies: Essays on theory and practice. Champaign, IL: Human Kinetics.

Hargreaves, J. (1986). Sport, power and culture: A social and historical analysis of popular sports in Britain. Cambridge, UK: Polity Press.

Harvey, L. (1990). Critical social research. London: Unwin Hyman. 
Harvey, J., \& Sparks, R. (1991). The politics of the body in the context of modernity. Quest, 43, 164-189.

Heikkala, J. (1993). Discipline and excel: Techniques of the self and body and the logic of competing. Sociology of Sport Journal, 10, 397-412.

Hickey, A., \& Austin, J. (2007). Pedagogies of self: Conscientising the personal to the social. International Journal of Pedagogies and Learning, 3(1), 21-29.

Howe, D. (2004). Sport, professionalism and pain: Ethnographies of injury and risk. London: Routledge.

Jackson, D. (1990). Unmasking masculinity. London: Unwin Hyman.

James, A. (2000). Embodied being(s): Understanding the self and body in childhood. In A. Prout (Ed.), The body, childhood and society (pp. 19-37). Basingstoke, UK: Palgrave MacMillan.

Johns, D., \& Johns, J. (2000). Surveillance, subjectivism and technologies of power. International Review for the Sociology of Sport, 35, 219-34.

Jones, R. (2000). Toward a sociology of coaching. In R. Jones \& K. Armour (Eds.), Sociology of sport: Theory and practice (pp. 33-43). London: Addison-Wesley-Longman.

Jones, R., Armour, K., \& Potrac, P. (2002). Understanding the coaching process: A framework for social analysis. Quest, 54, 34-48.

Jones, R., Glintmeyer, N., \& McKenzie, A. (2005). Slim bodies, eating disorders and the coach-athlete relationship: A tale of identity creation and disruption. International Review for the Sociology of Sport, 40(3), 377391.

Kirk, D. (1993). The body, schooling and culture. Geelong, Australia: Deakin University.

Kirk, D. (1999). Physical culture, physical education and relational analysis. Sport, Education and Society, 4(1), 63-75.

Kirk, D. (2002). The social construction of the body in physical education and sport. In A. Laker (Ed.), The sociology of sport and physical education (pp. 79-91). London: RoutledgeFalmer.

Kirk, D., \& Tinning, R. (1990). Physical education, curriculum and culture: Critical issues in the contemporary crisis. Hampshire, UK: Falmer Press.

Klein, N. (2000). No logo. London: Flamingo.

Lang, M. (2010). Surveillance and conformity in competitive youth swimming. Sport, Education and Society Journal, 15(1), 19-38.

Lee, J., \& Macdonald, D. (2010). Are they just checking our obesity or what?' The healthism discourse and rural young women. Sport, Education and Society Journal, 15(2), 203-219. 
Locke, L. (1985). Research and the improvement of teaching: The professor as the problem. In G. Barrette, R. Feingold, R. Rees \& M. Pieron (Eds.), Myths, models and methods in sport pedagogy (pp. 1-26). Champaign, IL:

Human Kinetics.

Lopiano, D., \& Zotos, C. (1992). Modern athletics: The pressure to perform. In K. Brownwell, J. Rodin \& J. Wilmore (Eds.), Eating, body weight, and performance in athletes: Disorders of modern society (pp. 275-292). Philadelphia, PA: Lea \& Febiger.

Lupton, D. (1996). Food, the body and the self. London: Sage.

Maglischo, E. (1993). Swimming fastest: The essential reference on technique, training and program design. Champaign, IL: Human Kinetics.

Maguire, J., \& Mansfield, L. (1998). No body's perfect: Women, aerobics and the body beautiful. Sociology of Sport Journal, 15, 109-137.

Markula, P. (1995). Firm but shapely, fit but sexy, strong but thin: The postmodern aerobicizing female bodies. Sociology of Sport Journal, 12, 424-453.

Markula, P. (2001). Beyond the perfect body: Women's body image distortion in fitness magazine discourse. Journal of Sport and Social Issues, 25, 158179.

Markula, P., \& Pringle, R. (2006). Foucault, sport and exercise: Power, knowledge and transforming the self. London: Routledge.

McMahon, J. (2007). Slim to win: An autoethnography from an Australian elite swimmer. Honours thesis, James Cook University, Cairns.

McMahon, J., \& Dinan-Thompson, M. (2008). A malleable body - Revelations from an Australian elite swimmer. Healthy Lifestyles Journal, 55(1), $23-$ 28.

McMahon, J., \& Dinan-Thompson, M. (in press). Body work - Regulation of a swimmer body: An autoethnography from an Australian elite swimmer. Sport, Education and Society.

McMahon, J., Penney, D., \& Dinan-Thompson, M. (in press). Body practices Exposure and effect? Stories from three Australian Swimmers. Sport, Education and Society.

McWilliam, E. (1999). Pedagogical pleasures. New York: Peter Lang.

Monoghan, L. (1997). We're not druggies, we're athletes: Bodybuilding, polypharmacology and self-identity. Unpublished $\mathrm{PhD}$ thesis, University of Wales, Cardiff.

O'Brien, J., Martin Ginis, K., \& Kirk, D. (2008). The effects of a body-focused physical and health education module on self-objectification and social 
physique anxiety in Irish girls. Journal of Teaching in Physical Education, 27, 116-126.

Patel, D., Greydanus, D., Pratt, H., \& Phillips, E. (2003). Eating disorders in adolescent athletes, Journal of Adolescent Research, 18, 280-296.

Patton, M. (2002). Qualitative Research and Evaluation Methods $\left(3^{\text {rd }}\right.$ ed.). Thousand Oaks, CA: Sage.

Pillow, W. (1997). Exposed methodology: The body as a deconstructive practice. Qualitative Studies in Education, 10(3), 349-363.

Polkinghorne, D. (1988). Narrative knowing and the human sciences. New York: State University of New York Press.

Prain, V., \& Hickey, C. (1995). Using discourse analysis to change physical education. Quest, 47, 76-90.

Prequel. (2009). In Merriam-Webster online dictionary. Retrieved September 7, 2009, from http://www.merriam-webster.com/dictionary/Prequel

Pringle, R. (2008). No rugby - no fear: Collective stories, masculinities and transformative possibilities in schools. Sport, Education and Society, 13(2), 215-237.

Prout, A. (2000). The body, childhood and society. Basingstoke, UK: Palgrave MacMillan.

Purdy, L., Potrac, P., \& Jones, R. (2008). Power, consent and resistance: An autoethnography of competitive rowing. Sport, Education and Society, 13(3), 319-336.

Pynchon, T. (1975). V. London: Picador.

Rail, G., \& Harvey, J. (1995). Body at work: Michel Foucault and the sociology of sport. Sociology of Sport Journal, 12, 164-179.

Riessman, C. (1993). Narrative analysis. Newbury Park, CA: Sage.

Rich, E. (2003). Exploring constructions of the body, (ill)health and identity in schools: The case of anorexia nervosa. Retrieved May 30, 2007, from http://www.inter-disciplinary.net/ptb/mso/hid/hid2/rich\%20paper.pdf

Rich, E. (2010). Body pedagogies, education and health. Sport, Education and Society, 5(2), 147-150.

Rich, E. \& Evans, J. (2007, December). Rereading voice: Young women, anorexia and performative education. Junctures: The Journal for Thematic Dialogue. Available from http://www.junctures.org/issues.php?issue $=09 \&$ title $=$ Voice $\&$ colour $=r g b(1$ $76,153,0)$

Rich, E., Holroyd, R., \& Evans, J. (2004). Hungry to be noticed: Young women, anorexia and schooling. In J. Evans, B. Davies \& J. Wright (Eds.), Body 
knowledge and control: Studies in the sociology of physical education and health (pp. 173-190). London: Routledge.

Richardson, L. (1990). Writing strategies: Reaching diverse audiences. Qualitative Research Methods, 21, 5-71.

Richardson, L. (1994). Writing: A method of inquiry. In N. K. Denzin \& Y. S. Lincoln (Eds.), Handbook of qualitative research (pp. 516-529).

Thousand Oaks, CA: Sage.

Richardson, L. (1995). Writing stories: Co-authoring the 'the sea monster,' a writing story. Qualitative Inquiry, 1, 189-203.

Richardson, L. (1997). Fields of play: Constructing an academic life. New Brunswick, NJ: Rutgers University Press.

Richardson, L. (2000). Writing: A method of inquiry. In N. K. Denzin \& Y. S. Lincoln (Eds.), Handbook of qualitative research (pp. 923-948). Thousand Oaks, CA: Sage.

Ricoeur, P. (1981). Narrative time. In W. Mitchell (Ed.), On narrative (pp. 165186). Chicago: University of Chicago Press.

Rinehart, R. (1998). Born-again sport: Ethics in biographical research. In G. Rail (Ed.), Sport and postmodern times. Albany, NY: University of New York Press.

Roberts, T. (1998). Private autonomy and public morality in sporting practices. In M. McNamee \& S. Parry (Eds.), Ethics and sport (pp. 240-255). London: Routledge.

Robinson, J., \& Hawpe, L. (1986). Narrative thinking as a heuristic process. In T. Sarbin (Ed.), Narrative psychology: The storied nature of human conduct (pp. 111-125). New York: Praeger.

Rodin, J., \& Larson, L. (1992). Social factors and the ideal body shape. In K. Brownwell, J. Rodin \& J. Wilmore (Eds.), Eating, body weight, and performance in athletes: Disorders of modern society (pp. 146-158). Philadelphia, PA: Lea \& Febiger.

Ropers-Huilman, B. (1999). Witnessing: Critical inquiry in a poststructural world. Qualitative Studies in Education, 12(1), 21-35.

Rorty, R. (1992). A pragmatist view of rationality and cultural difference. Philosophy East and West, 42(4), 581-596.

Sarbin, T. (1986). Narrative psychology: The storied nature of human conduct. New York: Praeger.

Seidman, I. (1998). Interviewing as qualitative research: A guide for researchers in education and the social science ( $2^{\text {nd }}$ ed.). New York: Teachers College Press. 
Sequel. (2009). In Merriam-Webster online dictionary. Retrieved September 7, 2009, from http://www.merriam-webster.com/dictionary/sequel

Sheridan, A. (1980). Michel Foucault: The will to truth. London: Tavistock.

Shilling, C. (1993). The body and social theory. London: Sage.

Shilling, C. (2004). Educating bodies: Schooling and the constitution of society. In J. Evans, B. Davies \& J. Wright (Eds.), Body, knowledge and control: Studies in the sociology of physical education and health (pp. $\mathrm{xv}-\mathrm{xxii}$ ). London: Routledge.

Shilling, C. (2005). Body pedagogics: A programme and paradigm for research. Paper presented to the School of Sport and Exercise Sciences, University of Loughborough, UK.

Shilling, C. (2007). Embodying sociology: Retrospect, progress and prospects. London: Blackwell.

Shilling, C. (2010). Exploring the society-body-school nexus: Theoretical and methodological issues in the study of body pedagogics. Sport, Education and Society, 15(2), 151-167.

Shogan, D. (1999). The making of high performance athletes: Discipline, diversity, and ethics. Toronto, Canada: University of Toronto Press.

Smith, B. (1999). The abyss: Exploring depression through a narrative of the self. Qualitative Inquiry, 5(2), 264-279.

Somekh, B. (2006). Constructing intercultural knowledge and understanding through collaborative action research. Teachers and Teaching: Theory and Practice, 12(1), 87-106.

Sparkes, A. (1997). Ethnographic fiction and representing the absent other. Sport, Education and Society, 2(1), 25-40.

Sparkes, A. (1999). Exploring body narratives. Sport, Education and Society, 4(1), 17-30.

Sparkes, A. (2004). From performance to impairment: A patchwork of embodied memories. In J. Evans, B. Davies, \& J. Wright (Eds.), Body knowledge and control: Studies in the sociology of physical education and health (pp. 140-155). London: Routledge.

Stanley, L. (1993). On auto/biography in sociology. Sociology, 27(1), 41-52.

Strean, W. (1998). Possibilities for qualitative research in sport psychology. The Sport Psychologist, 12, 333-345.

Sundgot-Borden, J. (1994). Eating disorders in female athletes. Sports Medicine, $17,176-188$.

Sutton-Smith, B. (1989). Introduction to play as performance, rhetoric and metaphor. Play and Culture, 2, 47-189. 
Taub, D., \& Benson, R. (1992). Weight concerns, weight control techniques, and eating disorders among adolescent competitive swimmers: The effect of gender. Sociology of Sport, 9, 76-86.

Tedlock, B. (1991). From participant observation to the observation of participation: The emergence of narrative ethnography. Journal of Anthropological Research, 47, 69-94.

Tedlock, B. (2005). Ethnography and ethnographic representation. In N. K. Denzin \& Y. S. Lincoln (Eds.), Handbook of qualitative research (pp. 455-486). Thousand Oaks, CA: Sage.

Theberge, N. (1987). Sport and women's empowerment. Women's Studies International Forum, 10, 387-393.

Theberge, N. (1991). Reflections on the body in the sociology of sport. Quest, 43, $148-167$.

Tinning, R. (1985). Physical education and the cult of slenderness. ACHPER National Journal, 107, 10-13.

Tinning, R. (1997). Performance and participation discourses in human movement: Towards a socially critical physical education. In J. FernandezBalboa (Ed.), Critical postmodernism in human movement, physical education and sport (pp. 99-121). Albany, NY: State University of New York Press.

Tinning, R., McCuaig, L., \& lisahunter. (2006). Teaching health and physical education in Australian schools. French's Forest, Australia: Pearson.

Trinh, T. (1999). Write your body: The body in theory. In J. Price \& M. Shildrick (Eds.), Feminist theory and the body: A reader (pp. 258-266). New York: Routledge.

Tsang, T. (2000). Let me tell you a story. Sociology of Sport journal, 17(10), 4459.

Turner, B. (2000). The Blackwell companion to social theory. Blackwell Publishing: Victoria, Australia.

Vertinsky, P. (1985). Risk benefit analysis of health promotions: Opportunities and threats for physical education. Quest, 37(1), 71-83.

Vertinsky, P. (2004). Locating a sense of place: Space, place and gender in the gymnasium. In P. Vertinsky \& J. Bale (Eds.), Sites of sport: Space, place, experience (pp. 8-25). London: Routledge.

Vigarello, G. (1978). The straightened-up body. Paris: Editions Universities.

Wainwright, S., \& Turner, B. (2004). Narratives of embodiment. In H. Thomas \& J. Ahmed (Eds.), Cultural bodies (pp. 98-120). Oxford, UK: Blackwell. 
Wainwright, S., Williams, C., \& Turner, B. (2007). Varieties of habitus and the embodiment of ballet. Qualitative Research, 6(4), 535-558.

Watson, C. (2009). Teachers are meant to be orthodox: Narrative and counter narrative in the discursive construction of identity in teaching. International Journal of Qualitative Studies in Education, 22(4), 469-483.

Welty, E. (1984). One writer's beginnings. Cambridge, MA: Harvard University Press.

Willis, P. (1977). Learning to labour. Farnborough, UK: Saxon House.

Wilmore, J., \& Costill, D. (1992). Nutrition and human performance. In K. Brownell, J. Rodin \& J. Wilmore (Eds.), Eating body weight and performance in athletes: Disorders of modern society (pp. 61-76). Philadelphia, PA: Lea \& Febiger.

Wright. J. (2000). Bodies, meanings and movement: A comparison of the language of a physical education lesson and a feldenkrais movement class. Sport, Education and Society, 5, 35-49.

Wright, J., \& Harwood, V. (2007, January 26-27). Bio-power, bio-pedagogies and the obesity epidemic. Bio-pedagogies conference, Centre for Interdisciplinary Youth Research, University of Wollongong, Australia.

Yates, A., Shisslak, C., Crago, M., \& Allender, J. (1994). Overcommitment to sport: Is there a relationship to the eating disorders? Clinical Journal of Sport Medicine, 4, 39-46.

Zembylas, M. (2007). Emotional ecology: The intersection of emotional knowledge and pedagogical content knowledge in teaching. Teaching and Teacher Education, 23(4), 355-367. 


\section{APPENDIXES}


APPENDIX A:

ETHICAL APPROVAL 


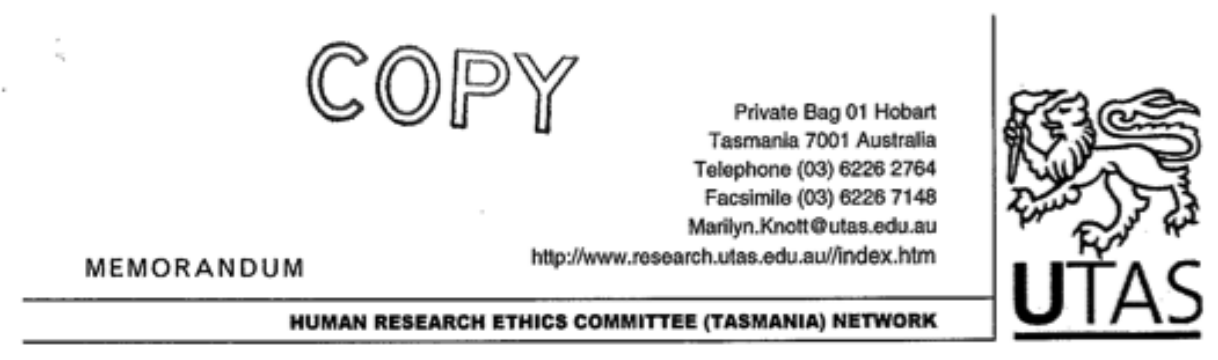

FULL COMMITTEE ETHICS APPLICATION APPROVAL

17 December 2008

AvProf Dawn Penney
Education
Private Bag 1308
Launceston

Ethics reference: $\mathrm{H} 10376$

'Narratives of the body, self and pedagogy'.

PhD candidate: Jenny McMahon

Dear Dr Penney

The Tasmania Social Sciences HREC Ethics Committee approved the above project on 17 December 2008.

All committees operating under the Human Research Ethics Committee (Tasmania) Network are registered and required to comply with the National Statement on Ethical Conduct in Human Research (NHMRC 2007).

Therefore, the Chief Investigator's responsibility is to ensure that:

1) All researchers listed on the application comply with HREC approved application.

2) Modifications to the application do not proceed until approval is obtained in writing from the HREC.

3) The confidentiality and anonymity of all research subjects is maintained at all times, except as required by law.

4) Statement 5.5.3 of the National Statement states:

Researchers have a signilicant responsibility in monitoring approved research as they are in the best position to observe any adverse events or unexpected outcomes. They should report such events or outcomes promptly to the relevant institution/s and ethical review bodyfies and take prompt steps to deal with any unexpected risks.

5) All participants must be provided with the current Information Sheet and Consent form as approved by the Ethics Committee.

6) The Committee is notified if any investigators are added to, or cease involvement with, the project.

A PARTNERSHIP PROGRAM IN CONJUMCTION WITH THE DEPARTMENT OF HEALTH AND HUMAN SERVICES 
7) This study has approval for 4 years contingent upon annual review. A Progress Report is to be provided on the anniversary date of your approval. You will be sent a courtesy reminder closer to this due date.

8) A Final Report and a copy of the published material, either in full or abstract, must be provided at the end of project.

Yours sincerely

a. knatt

for Ethics Executive Otticer

A PARTNERSHIP PROGRAM IN CONJUNCTION WITH THE DEPARTMENT OF HEALTH AND HUMAN SERVICES 
APPENDIX B:

ETHICAL AMENDMENT 


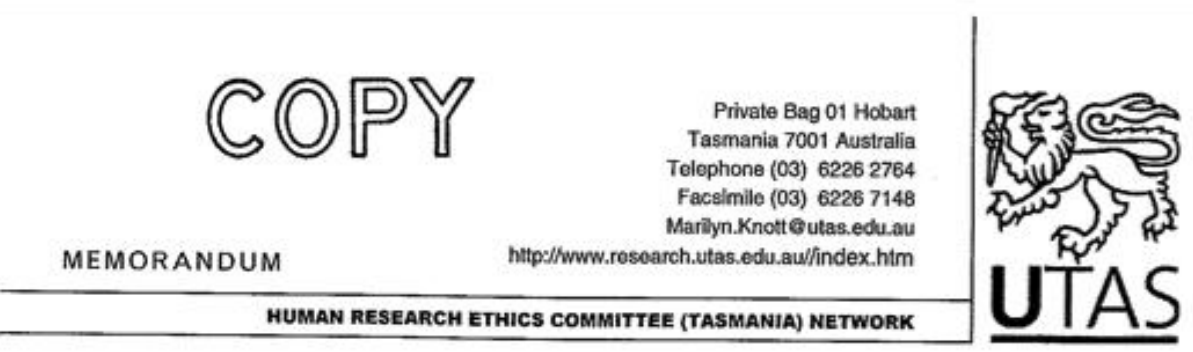

\section{AMENDMENT TO EXISTING APPLICATION APPROVAL}

18 February 2009

Dr Dawn Penney

Education

Private Bag 1308

Launceston

Ethics reference: $\mathbf{H} 10376$

'Narratives of the body, self and pedagogy'

PhD candidate: Jenny McMahon

Dear Dr Penney

The Chair of the Tasmania Social Sciences Human Research Ethics Committee approved the Amendment to the above project on 16 February 2009.

Amendment description:

Requested change in the number of participants from two to three people, but recruitment procedures will remain the same.

Full details as per formal Ethics Amendment form submitted for Ethics approval.

Yours sincerely<smiles>C=CCCCCC(C)(C)COCC</smiles>

Cor Ethics Executive OHlicer

Sor

A PARTNERSHIP PROGRAM IN CONUUNCTION WITH THE DEPARTMENT OF HEALTH ANO HUMAN SERVICES 
APPENDIX C:

INFORMATION SHEET 


\section{PARTICIPANT INFORMATION SHEET}

You are invited to participate in a research study that investigates coaching practices and the affect that these practices have on the body, self and post career on teaching/coaching practices? You were identified as an ideal candidate for this research as you are over 18 years of age and your experiences as an elite swimmer coach and/or teacher are pertinent to this research.

STUDY TITLE: Narratives of the body, self and pedagogy

Chief Investigators: Jenny McMahon, Associate Professor Dawn Penney, Dr Dean Cooley - University of Tasmania and Dr Maree DinanThompson - James Cook University

\section{Purpose of the study?}

This study will identify the coaching practices experienced by three elite swimmers during their adolescence and how these practices, if at all, have affected their body and self. The study will also identify whether the coaching practices experienced by the three participants as adolescent athletes has later affected/influenced their own pedagogy in their chosen profession. The study will do this by pulling together stories from the two participants.

\section{Why have I been invited to participate in this study?}

You are eligible to participate in this study because you are over 18 years of age. You are also an elite swimmer who represented Queensland and/or Australia during your adolescence and have later chosen a career in a performative culture where pedagogical practice (principles and methods of instruction) is essential.

\section{Participation is voluntary?}

Your participation in this research is voluntary. You may decline to answer any or all of the questions, and you are free to withdraw at any time, without explanation. You may also withdraw any data that you have supplied prior to the date of your withdrawal. All information will be treated in a confidential manner, and your name will not be used in any publication arising out of the research. If you are involved in the focus groups, every precaution will be undertaken to ensure your identity is kept confidential from the main participants, however there is a minimal risk that your identity may be exposed. All of the research will be kept in a locked cabinet in the office of Associate Professor Dawn Penney.

\section{What does this study involve?}


You will be presented with no more than four [4] questions related to a particular theme over four [4] e-mail interviews. You will be asked to respond to the questions in a story format to help us better understand coaching practices and the impact of such practices. If required, follow up phone calls or face-to-face meetings may be needed for clarification.

\section{Are there any possible benefits from participation in this study?}

We are hoping to identify ways to improve coaching practices in the hope that new strategies can be applied benefiting future athletes on a short and long-term basis.

\section{Are there any possible risks from participation in this study?}

There are no specific risks anticipated with participation in this study. However, if you find that you are becoming distressed, we will arrange for you to see a counsellor through the University of Tasmania at no expense to you. If you are residing out of Tasmania, a telephone session will be provided.

\section{What if I have questions about this research?}

If you would like to discuss any aspect of this study please feel free to contact either Jenny McMahon on (03) 63243054 or via e-mail Jennifer.McMahon@ utas.edu.au or Associate Professor Dawn Penney on (03) 63243680 or via email

Dawn.Penney@utas.edu.au. Either of us would be happy to discuss any aspect of the research with you. Once we have analysed the information we will be mailing / emailing you a summary of our findings. You are welcome to contact us at that time to discuss any issue relating to the research study.

This study has been approved by the Tasmanian Health and Human Services Research Ethics Committee. If you have concerns or complaints about the conduct of this study should contact the Executive Officer of the HREC (Tasmania) Network on (03) 6226 7479 or e-mail human.ethics@utas.edu.au. The Executive Officer is the person nominated to receive complaints from research participants. You will need to quote [HREC project number].

Thank you for taking the time to consider this study. If you wish to take part in it, please sign the attached consent form. You are able to keep the information sheet. 


\section{APPENDIX D:}

CONSENT FORM 
Locked Bag 1330, Launceston

Tasmania, 7250, Australia

Telephone: (03) 63243512 Facsimile: (03) 63243679

\section{CONSENT FORM}

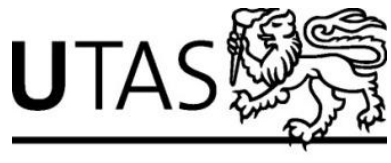

FACULTY OF EDUCATION

School of Education

Title of Project: Narratives of the body, self and pedagogy

1. I have read and understood the 'Information Sheet' for this project.

2. The nature and possible effects of the study have been explained to me.

3. I understand that the study involves an interview over four [4] e-mails with no more than four [4] questions related to a particular theme per e-mail. I will be required to respond to the questions in a short story format. If required, follow up phone calls and face to face consultations may occur.

4. I understand that participation involves the risk(s) where I may experience discomfort where I am asked to recall my experiences as an athlete. Any risk attached to this is mitigated by the researcher treating data pertaining to me confidentially and additionally offering counselling if need be. In any event, I understand that my participation is entirely voluntary and that I can withdraw from the study at any time.

5. I understand that all research data will be securely stored on the University of Tasmania premises for five years [or at least five years], and will then be destroyed [or will be destroyed when no longer required].

6. Any questions that I have asked have been answered to my satisfaction.

7. I agree that research data gathered from me for the study may be published provided that I cannot be identified as a participant.

8. I understand that the researchers will take every precaution to maintain my identity confidential, however there is a minimal risk that your identity may be exposed. Any information I supply to the researcher(s) will be used only for the purposes of the research.

9. I agree to participate in this investigation and understand that I may withdraw at any time without any effect, and if I so wish may request that any data I have supplied to date be withdrawn from the research.

Name of Participant:

Signature:

Date:

\section{Statement by Investigator}

I have explained the project \& the implications of participation in it to this volunteer and I believe that the consent is informed and that he/she understands the implications of participation

If the Investigator has not had an opportunity to talk to participants prior to them participating, the following must be ticked. 
The participant has received the Information Sheet where my details have been provided so participants have the opportunity to contact me prior to consenting to participate in this project.

Name of Investigator

Signature of

Investigator

Name of investigator

Signature of investigator Date 
APPENDIX E:

COUNSELLING ADVICE 
Hi Jenny,

My sincere apologies in getting back to you so late. I have had a chat with Stephanie Taylor, Manager of Student Services, regarding your request as I am leaving in a couple of weeks. We have agreed that we could offer any of the study participants a single session at no cost to debrief, and if they need further assistance, to refer to an appropriate agency depending on the issues they present with. Unfortunately we do not currently have sufficient counselling resources to offer more than this. Do you think this will be adequate for your needs?

Regards,

Kelly Parkyn

Team Leader

Counselling and Disability

Student Services

University of Tasmania

Private Bag 2

Hobart TAS 7001

Ph 62262697

Work Days: Mon to Thu 9-2.30, Friday 9-5

CRICOS Provider Code: 00586B 